\title{
A $C$-Glycosidation Approach to the Central Core of Amphidinol 3: Synthesis of the C39-C52 Fragment
}

\author{
Javier de Vicente, Bodo Betzemeier, and Scott D. Rychnovsky*
}

Department of Chemistry, 516 Rowland Hall, University of California, Irvine, California 926972025

\section{Table of Contents}

1 General Methods 1

2 Synthesis of the C39-C52 Fragment of Amphidinol 3 (3) ............................ 3

3 Reductive Lithiations of Phenylthio Acetals 5 19

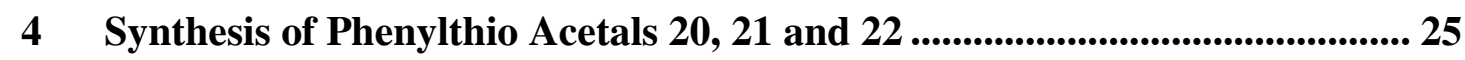

5 Reductive Lithiation of Phenylthio Acetals 20, 21 and 22 ......................... 33

6 Conformational Analysis of Model Pyranosyl Radical Intermediate 26 ..... 41

\section{General Methods}

General experimental details: All reactions were carried out under an atmosphere of Ar using oven or flame dried glassware and standard syringe/septa techniques. Solvent removal was performed under reduced pressure using a Büchi rotary evaporator. All reactions were stirred with a Teflon- or Pyrex-coated stir-bar and magnetic stir-plate. Tetrahydrofuran (THF), $\mathrm{Et}_{2} \mathrm{O}$, $\mathrm{CH}_{2} \mathrm{Cl}_{2}$ (DCM), and toluene were degassed with argon and then passed through two $4 \times 36$ inch columns of anhydrous neutral alumina A-2 alumina $(8 \times 14$ mesh; LaRoche Chemicals; 
activated under a flow of Ar at $350{ }^{\circ} \mathrm{C}$ for $12 \mathrm{~h}$ to remove $\mathrm{H}_{2} \mathrm{O}$, according to the procedure described by Grubbs. ${ }^{1}$ Triethylamine $\left(\mathrm{Et}_{3} \mathrm{~N}\right)$, pyridine, and diisopropylethylamine $\left(i-\mathrm{Pr}_{2} \mathrm{NEt}\right)$ were distilled from $\mathrm{CaH}_{2}$ at atmospheric pressure. Ethylenediamine was distilled from sodium at atmospheric pressure. Indicated molarities of organolithium reagents were determined by titration with either menthol and 1,10-phenanthroline or $N$-benzylbenzamide. ${ }^{2}$ All chemicals were purchased from Aldrich or Acros and used as delivered. Aldehyde 7 was synthesized using a modified procedure described by Mukaiyama. ${ }^{3}$ Epoxy aldehyde 6 was synthesized according to the procedure described by Sharpless. ${ }^{4}$

Instrumentation and chromatography: $400 \mathrm{MHz}{ }^{1} \mathrm{H}$ and $100 \mathrm{MHz}{ }^{13} \mathrm{C}$ spectra were obtained on a Brüker DRX $400 \mathrm{FT}$ NMR; $500 \mathrm{MHz}{ }^{1} \mathrm{H}$ and $125 \mathrm{MHz}{ }^{13} \mathrm{C}$ NMR spectra were obtained on a Brüker GN 500 FT NMR or Brüker Nicolet $\Omega 500$ FT NMR. ${ }^{1} \mathrm{H}$ NMR chemical shifts are reported as $\delta$ values in ppm to the nearest one-hundredth. Chemical shifts of the ${ }^{1} \mathrm{H}$ and ${ }^{13} \mathrm{C}$ NMR spectra were referenced to residual solvent peaks. ${ }^{5}$ Coupling constants are reported in $\mathrm{Hz}$ to the nearest one-tenth and refer to apparent multiplicities. Multiplicity is indicated as follows: s (singlet); br s (broad singlet); d (doublet); $\mathrm{t}$ (triplet); m (multiplet); app t (apparent triplet); dd (doublet of a doublet); ddd (doublet of a doublet of a doublet); etc. Mass spectra were measured on a MicroMass Analytical 7070E (CI-isobutane), a MicroMass AutoSpec E (FAB) spectrometer, or a Micromass LCT Electrospray spectrometer. Infrared spectra were recorded on a MIDAC Prospect FT-IR spectrometer. Combustion analyses were performedby M-H-W Laboratories (Phoenix, AZ). Optical rotations were measured using a JASCO DIP-370 digital polarimeter. Liquid chromatography was performed using forced flow (flash chromatography) of the indicated solvent system on E. Merck reagent silica gel 60 (230400 mesh).

\footnotetext{
${ }^{1}$ Pangborn, A. B.; Giardello, M. A.; Grubbs, R. H.; Rosen, R. K.; Timmers, F. J. Organometallics 1996, 15, 1518 .

${ }^{2}$ Burchat, A. F.; Chong, J. M.; Nielsen, N. J. Organomet. Chem. 1997, 542, 281.

${ }^{3}$ Mukaiyama, T.; Suzuki, K.; Yamada, T.; Tabusa, F. Tetrahedron 1990, 46, 265.

${ }^{4}$ Gao, Y.; Hanson, R. M.; Klunder, J. M.; Ko, S. Y.; Masamune, H.; Sharpless, K. B. J. Am. Chem. Soc. 1987, 109, 5765 .

${ }^{5}$ Gottieb, H. E.; Kotlyar, V.; Nudelman, A. J. Org. Chem. 1997, 62, 7512.
} 


\section{Synthesis of the C39-C52 Fragment of Amphidinol 3 (3)}
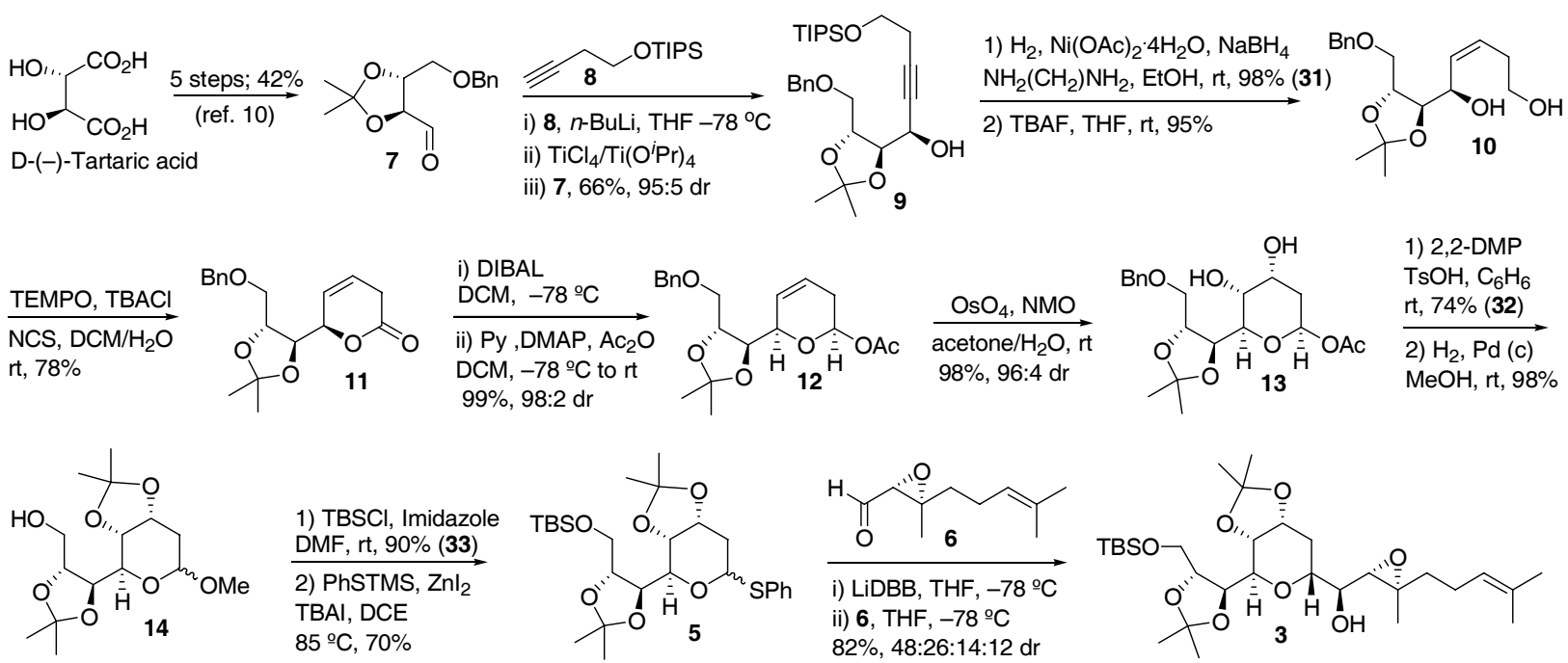

\section{(3-Butynyloxy)(triisopropyl)silane (8)}

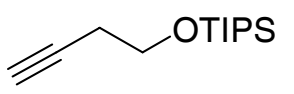

Anhydrous DMF (100 mL) was added to a flame dried flask containing imidazole (35 g, $514 \mathrm{mmol})$ and DMAP $(1.3 \mathrm{~g}, 10.6 \mathrm{mmol})$ at rt. Butyn-1-ol $(15.9 \mathrm{ml}, 210 \mathrm{mmol})$ and triisopropylsilyl chloride $(53.8 \mathrm{~mL}, 252 \mathrm{mmol})$ were then sequentially added. After stirring at $\mathrm{rt}$ for $16 \mathrm{~h}$, the reaction was quenched by addition of water $(500 \mathrm{~mL})$ and diluted with hexanes $(500 \mathrm{~mL})$. The organic phase was separated and the aqueous was extracted with hexanes $(3 \times$ $150 \mathrm{~mL}$ ). The combined organic phases were dried over $\mathrm{MgSO}_{4}$, filtered and evaporated under reduced pressure to give the crude oil. This oil was purified by column chromatography (hexanes) to give silyl ether $\mathbf{8}$ as a colorless oil $(43.8 \mathrm{~g}, 93 \%) . \mathrm{R}_{\mathrm{f}}=0.35$ (eluent hexanes). ${ }^{1} \mathbf{H}$ NMR (400 MHz, $\left.\mathrm{CDCl}_{3}\right) \delta 3.83(\mathrm{~d}, J=7.2 \mathrm{~Hz}, 2 \mathrm{H}), 2.44(\mathrm{td}, J=7.2$ and $2.5 \mathrm{~Hz}, 2 \mathrm{H}), 1.96$ (t, $J=2.5 \mathrm{~Hz}, 1 \mathrm{H}), 1.08(\mathrm{~s}, 21 \mathrm{H}) ;{ }^{13} \mathrm{C}$ NMR (100 MHz, $\left.\mathrm{CDCl}_{3}\right) \delta$ 81.5, 62.0, 22.9, 17.9 (6 C), 12.3 (3 C); IR $v_{\max } / \mathrm{cm}^{-1} 3314,2958,2944,2868,1464,1115,882,681,635$. LRMS (EI): $\mathrm{m} / z$ $183(\mathrm{M}-i \mathrm{Pr})^{+} . \mathbf{C}_{\mathbf{1 3}} \mathbf{H}_{\mathbf{2 6}} \mathbf{O S i}(\mathrm{M}=226.43 \mathrm{~g} / \mathrm{mol})$ calcd C: $68.96, \mathrm{H}: 11.57$, found C: $68.78, \mathrm{H}$ : 11.67. 
(1R)-1-(4R,5R)-5-[(Benzyloxy)methyl]-2,2-dimethyl-1,3-dioxolan-4-yl-5-[(1,1,1triisopropylsilyl)oxy]-2-pentyn-1-ol (9)

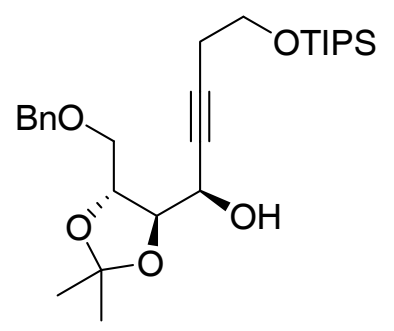

A solution of $n$-BuLi (2.4 M in hexanes, $46 \mathrm{~mL}, 110.3 \mathrm{mmol}$ ) was slowly added to a solution of alkyne $8(28.2 \mathrm{~g}, 124.5 \mathrm{mmol})$ in anhydrous THF $(700 \mathrm{~mL})$ at $-78^{\circ} \mathrm{C}$. The mixture was stirred at $-78{ }^{\circ} \mathrm{C}$ for $15 \mathrm{~min}$. A brown solution of $\mathrm{Ti}(i-\mathrm{PrO})_{4}(16.5 \mathrm{~mL}, 54.7 \mathrm{mmol})$ and $\mathrm{TiCl}_{4}(1 \mathrm{M}$ solution in DCM, $55 \mathrm{~mL}, 55 \mathrm{mmol}$ ) was then added dropwise to the reaction mixture and the resultant mixture was stirred at $-78^{\circ} \mathrm{C}$ for $1.5 \mathrm{~h}$. A solution of aldehyde $7(24 \mathrm{~g}, 95.9$ mmol) in anhydrous THF $(350 \mathrm{~mL})$ was added over a period of $1 \mathrm{~h}$. The resultant mixture was stirred at $-78^{\circ} \mathrm{C}$ for $5 \mathrm{~h}$ and then slowly warmed to rt overnight. The reaction was quenched by addition of a $0.05 \mathrm{M}$ phosphate buffer $\mathrm{pH}=7(200 \mathrm{~mL})$ and filtered through a celite $®$ plug. The filtrate was evaporated under reduced pressure to give a mixture of an orange oil and an aqueous solution. Ether $(200 \mathrm{~mL})$ and water $(100 \mathrm{~mL})$ were added. The orange organic phase was separated and washed with brine $(100 \mathrm{~mL})$. The organic phase was then separated, dried over $\mathrm{MgSO}_{4}$, filtered and evaporated under reduced pressure to give a yellow oil. The crude material was purified by column chromatography (hexanes/ether 2:1) to give alcohol $9(30.1 \mathrm{~g}, 66 \%)$ as an orange oil in a 95:5 diastereomeric ratio in favour of the $R$ alcohol. $\mathrm{R}_{\mathrm{f}}=0.25$ (eluent hexanes/ether 2:1) (vide infra). $[\boldsymbol{\alpha}]^{\mathbf{2 0}}{ }_{\mathbf{D}}+7.34\left(\mathrm{c} 0.49, \mathrm{CHCl}_{3}\right) .{ }^{\mathbf{1}} \mathbf{H} \mathbf{~ N M R}\left(500 \mathrm{MHz}, \mathrm{CDCl}_{3}\right) \delta$ 7.33-7.31 (m, $4 \mathrm{H}), 7.26(\mathrm{~m}, 1 \mathrm{H}), 4.59(\mathrm{~s}, 2 \mathrm{H}), 4.50(\mathrm{tt}, J=4.4$ and $2.0 \mathrm{~Hz}, 1 \mathrm{H}), 4.25$ (ddd, $J$ $=8.1,5.5$ and $4.0 \mathrm{~Hz}, 1 \mathrm{H}), 3.95(\mathrm{dd}, J=8.0$ and $4.1 \mathrm{~Hz}, 1 \mathrm{H}), 3.75(\mathrm{t}, J=7.4 \mathrm{~Hz}, 2 \mathrm{H}), 3.70$ $(\mathrm{dd}, J=10.3$ and $4.0 \mathrm{~Hz}, 1 \mathrm{H}), 3.64(\mathrm{dd}, J=10.3$ and $5.5 \mathrm{~Hz}, 1 \mathrm{H}), 2.47(\mathrm{~d}, J=4.6 \mathrm{~Hz}, 1 \mathrm{H})$, $2.39(\mathrm{tt}, J=7.4$ and $2.0 \mathrm{~Hz}, 2 \mathrm{H}), 1.44(\mathrm{~s}, 3 \mathrm{H}), 1.43(\mathrm{~s}, 3 \mathrm{H}), 1.08-1.02(\mathrm{~m}, 21 \mathrm{H}) .{ }^{\mathbf{1 3}} \mathbf{C} \mathbf{~ N M R}$ $\left.125 \mathrm{MHz}, \mathrm{CDCl}_{3}\right) \delta 137.8,128.4$ (2 C), 127.7, 127.6 (2 C), 109.8, 84.5, 80.1, 78.1, 76.5, 73.5, 70.9, 62.5, 61.9, 27.2, 26.9, 23.1, 17.9 (6 C), 11.9 (2 C); IR $v_{\max } / \mathrm{cm}^{-1} 3455,2942,2867,1463$, 1381, 1248, 1216, 1107, 882; MS (EI): $m / z 500(\mathrm{M}+\mathrm{H}+\mathrm{Na})^{+}$; HRMS (EI): $\mathrm{m} / z$ calcd for $\mathrm{C}_{27} \mathrm{H}_{44} \mathrm{NaO}_{5} \mathrm{Si}:$ 499.2656, found 499.2857. $\mathbf{C}_{27} \mathbf{H}_{44} \mathbf{O}_{5} \mathbf{S i}(\mathrm{M}=476.72 \mathrm{~g} / \mathrm{mol})$ calcd C: $68.02, \mathrm{H}$ : 9.30 found calcd C: $68.10, \mathrm{H}: 9.40$. 
$(1 R, 2 Z)-1-(4 R, 5 R)-5-[(B e n z y l o x y) m e t h y l]-2,2-d i m e t h y l-1,3-d i o x o l a n-4-y l-5-[(1,1,1-$ triisopropylsilyl)oxy]-2-penten-1-ol (31)

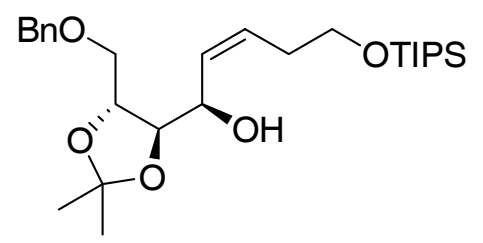

Solid $\mathrm{NaBH}_{4}\left(734 \mathrm{mg}, 19.7 \mathrm{mmol}\right.$ ) was added to a degassed solution of $\mathrm{Ni}(\mathrm{OAc})_{2} \bullet \mathrm{H}_{2} \mathrm{O}$ (4.9 g, $19.7 \mathrm{mmol})$ in ethanol $(993 \mathrm{~mL})$. The mixture was then purged with $\mathrm{H}_{2}$. Ethylenediamine (2.63 $\mathrm{mL}, 39.35 \mathrm{mmol})$ was added and the black resultant colloid was stirred at $\mathrm{rt}$ for $5 \mathrm{~min}$. A solution of alkyne $9(28.4 \mathrm{~g}, 59.6 \mathrm{mmol})$ in degassed ethanol $(60 \mathrm{~mL})$ was then added. The mixture was stirred at $\mathrm{rt}$ under $\mathrm{H}_{2}$ (ca. 1 atm) until no starting material was observed by ${ }^{1} \mathrm{H} \mathrm{NMR}$ analysis of aliquots taken from the reaction mixture (normally $3 \mathrm{~h}$ ). The reaction mixture was purged with argon and the solvent was removed under reduced pressure. The crude oil was dissolved in ether $(300 \mathrm{~mL})$ and washed with brine $(200 \mathrm{~mL})$. The aqueous phase was extracted with ether $(3 \times 100 \mathrm{~mL})$. The combined organic extracts were dried over $\mathrm{MgSO}_{4}$, filtered and evaporated under reduced pressure to afford alkene $31(27 \mathrm{~g}, 95 \%)$ as a colorless oil: $\mathrm{R}_{\mathrm{f}}=0.25$

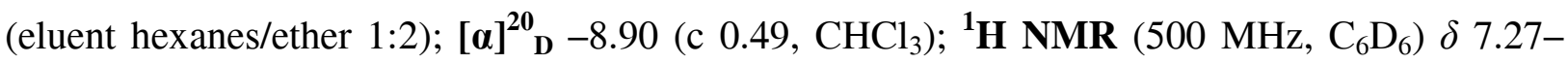
7.25 (m, 2 H), 7.17-7.15 (m, 2 H), 7.09-7.07 (m, 1 H), 5.75-5.71 (m, 1 H), 5.64-5.59 (m, $1 \mathrm{H})$, $4.60(\mathrm{t}, J=6.7 \mathrm{~Hz}, 1 \mathrm{H}), 4.38$ and 4.35 (AB system, $J=12.1 \mathrm{~Hz}, 2 \mathrm{H}), 4.29$ (dt, $J=7.9$ and 4.6 $\mathrm{Hz}, 1 \mathrm{H}), 4.01(\mathrm{dd}, J=7.9$ and $6.0 \mathrm{~Hz}, 1 \mathrm{H}), 3.65(\mathrm{dd}, J=10.2$ and $4.4 \mathrm{~Hz}, 1 \mathrm{H}), 3.62-3.54(\mathrm{~m}$, 3 H), 2.59 (br s, 1 H), 2.46-2.39 (m, 1 H), 2.28-2.22 (m, 1 H), 1.40 (s, 3 H), 1.39 (s, 3 H), 1.111.09 (m, $21 \mathrm{H}) ;{ }^{13} \mathrm{C}$ NMR $\left(125 \mathrm{MHz}, \mathrm{C}_{6} \mathrm{D}_{6}\right) \delta$ 138.8, 131.6, 130.3, 128.7 (2 C), 127.7 (2 C), 128.1, 109.2, 81.5, 78.6, 73.7, 71.6, 68.8, 63.1, 32.2, 27.3 (2 C), 18.3 (6 C), 12.4 (2 C); IR $v_{\max } / \mathrm{cm}^{-1} 3455,2941,2865,1462,1380,1248,1214,1100,882 ; \mathbf{M S ~ m} / \mathrm{z}(\mathrm{EI}) \mathrm{m} / \mathrm{z} 463$ (M $\left.\mathrm{CH}_{3}\right)^{+}$; HRMS (EI): $\mathrm{m} / z$ calcd for $\mathrm{C}_{26} \mathrm{H}_{43} \mathrm{O}_{5} \mathrm{Si}: 463.2880$, found 463.2889. $\mathbf{C}_{\mathbf{2 7}} \mathbf{H}_{46} \mathbf{O}_{5} \mathbf{S i}(\mathbf{M}=$ $478.74 \mathrm{~g} / \mathrm{mol}$ ) calcd C: 67.74, H: 9.68 found C: 67.90, H: 9.52. 
Mosher ester analysis of allylic alcohol 31

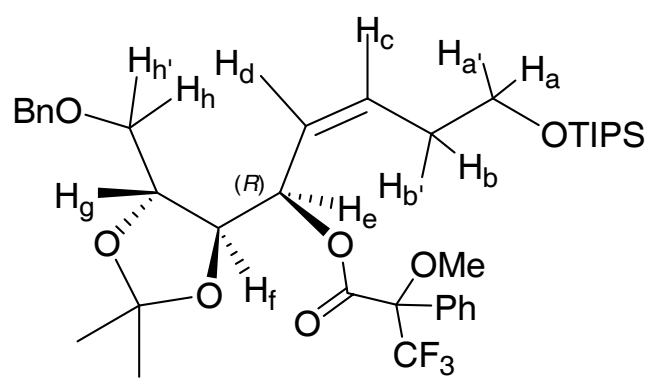

\begin{tabular}{cccc}
\hline & $\boldsymbol{\delta}(\boldsymbol{R}, \boldsymbol{R})$ & $\boldsymbol{\delta}(\boldsymbol{R}, \boldsymbol{S})$ & $\boldsymbol{\delta}(\boldsymbol{R}, \boldsymbol{S})-\boldsymbol{\delta}(\boldsymbol{R}, \boldsymbol{R})^{\mathbf{a}}$ \\
\hline H-a & 3.77 & 3.77 & 0 \\
H-a' & 3.72 & 3.72 & 0 \\
H-b & 2.5 & 2.53 & 0.03 \\
H-b' & 2.43 & 2.47 & 0.04 \\
H-c & 5.88 & 5.89 & 0.01 \\
H-d & 5.34 & 5.47 & 0.13 \\
H-e & - & - & - \\
H-f & 4.05 & 3.98 & -0.07 \\
H-g & 4.09 & 4.02 & -0.07 \\
H-h & 3.59 & 3.43 & -0.16 \\
H-h' & 3.55 & 3.35 & -0.2 \\
\hline
\end{tabular}

${ }^{\text {a }}$ Positive values for $\Delta \delta$ means that the signal is shifted downfield and negative values for $\Delta \delta$ means that the signal is shifted upfield from that of the other diastereomeric ester.

(1R,2Z)-1-(4R,5R)-5-[(Benzyloxy)methyl]-2,2-dimethyl-1,3-dioxolan-4-yl-2-pentene-1,5-diol (10)<smiles>CC1(C)O[C@@H](COBr)[C@H](C(O)/C=C\CCO)O1</smiles>

TBAF was added to a solution of silyl ether 31 (27 g, $56.44 \mathrm{mmol})$ in THF (30 mL) at rt. The reaction mixture was stirred at $\mathrm{rt}$ for $3 \mathrm{~h}$. Brine $(200 \mathrm{~mL})$ and ether $(200 \mathrm{~mL})$ were added. 
The aqueous phase was separated and washed with ether $(2 \times 100 \mathrm{~mL})$. The combined organic extracts were dried over $\mathrm{MgSO}_{4}$, filtered and evaporated under reduced pressure to give the crude material, which was purified by column chromatography (hexanes/ether 10:1) to afford diol $10(17.3 \mathrm{~g}, 95 \%)$ as a colorless oil. $\mathrm{R}_{\mathrm{f}}=0.3$ (eluent hexanes/ether $\left.1: 9\right)$; $[\boldsymbol{\alpha}]^{\mathbf{2 0}}{ }_{\mathrm{D}}+13.78$ (c 1.33, $\left.\mathrm{CHCl}_{3}\right) ;{ }^{1} \mathbf{H}$ NMR $\left(500 \mathrm{MHz}, \mathrm{C}_{6} \mathrm{D}_{6}\right) \delta$ 7.26-7.25 (m, $\left.2 \mathrm{H}\right), 7.17-7.12(\mathrm{~m}, 2 \mathrm{H}), 7.09-7.06$ (m, $1 \mathrm{H}), 5.78-5.75(\mathrm{~m}, 1 \mathrm{H}), 5.47-5.42(\mathrm{~m}, 1 \mathrm{H}), 4.55(\mathrm{t}, J=7.3 \mathrm{~Hz}, 1 \mathrm{H}), 4.36$ and 4.33 (AB system, $J=12.1 \mathrm{~Hz}, 2 \mathrm{H}), 4.25(\mathrm{dt}, J=7.9$ and $4.7 \mathrm{~Hz}, 1 \mathrm{H}), 3.97(\mathrm{dd}, J=7.8$ and $6.6 \mathrm{~Hz}, 1 \mathrm{H})$, $3.64(\mathrm{dd}, J=10.1$ and $4.7 \mathrm{~Hz}, 1 \mathrm{H}), 3.61(\mathrm{dd}, J=10.1$ and $4.8 \mathrm{~Hz}, 1 \mathrm{H}) 3.45$ (br s, $1 \mathrm{H}), 3.40$ 3.30 (m, 2 H), 2.55 (br s, 1 H), 2.24-2.17 (m, 1 H), 2.12-2.05 (m, 1 H), 1.40 (s, 3 H), 1.39 (s, 3 $\mathrm{H}) ;{ }^{13} \mathrm{C}$ NMR (125 MHz, $\left.\mathrm{C}_{6} \mathrm{D}_{6}\right) \delta$ 138.5, 132.7, 130.5, 128.7 (2 C), 128.0 (2 C), 127.9, 109.4, 81.5, 79.0, 73.7, 71.5, 68.5, 61.2, 31.7, 27.3, 27.2; IR $v_{\max } / \mathrm{cm}^{-1} 3408,2985,2872,1454,1380$, 1250, 1214, 1075, 858; MS m/z (EI) $m / z 307\left(\mathrm{M}-\mathrm{CH}_{3}\right)^{+}$; HRMS (EI): $m / z$ calcd for $\mathrm{C}_{17} \mathrm{H}_{23} \mathrm{O}_{5}$ : 307.1546, found 307.1540. $\mathbf{C}_{\mathbf{1 8}} \mathbf{H}_{\mathbf{2 6}} \mathbf{O}_{\mathbf{5}}(\mathrm{M}=322.40 \mathrm{~g} / \mathrm{mol})$ calcd $\mathrm{C}: 67.06, \mathrm{H}: 8.13$ found $\mathrm{C}$ : 67.38, H: 7.95 .

(6R)-6-(4S,5R)-5-[(Benzyloxy)methyl]-2,2-dimethyl-1,3-dioxolan-4-yl-3,6-dihydro-2H-2pyranone (11)

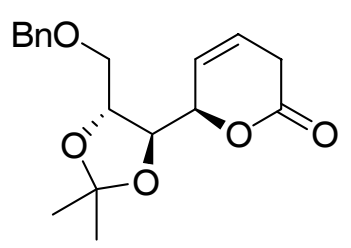

A mixture of diol 10 (6.42 g, $19.91 \mathrm{mmol})$, TEMPO (311 mg, $1.99 \mathrm{mmol})$ and TBACl $(553 \mathrm{mg}, 1.99 \mathrm{mmol})$ was dissolved in DCM $(124 \mathrm{~mL})$ and treated with a buffer carbonate solution $\mathrm{pH}=8.6\left(94 \mathrm{~mL}, \mathrm{NaHCO}_{3}(0.5 \mathrm{M}) / \mathrm{K}_{2} \mathrm{CO}_{3}(0.05 \mathrm{M})\right)$ at rt. A sample of NCS $(10.63 \mathrm{~g}$, $79.64 \mathrm{mmol}$ ) was then added portionwise over a period of $5 \mathrm{~min}$. The mixture was stirred at $\mathrm{rt}$ in the dark for $1 \mathrm{~h}$. The reaction was quenched with brine $(100 \mathrm{~mL})$ and extracted with ether $(2 \times$ $200 \mathrm{~mL}$ ). The organic phase was dried over $\mathrm{MgSO}_{4}$, filtered and evaporated under reduced pressure to give the crude material, which was purified by column chromatography (hexanes/ether 1:2) to afford lactone $11(5.06 \mathrm{~g}, 80 \%)$ as a colorless oil: $\mathrm{R}_{\mathrm{f}}=0.3$ (eluent hexanes/ether 1:2); $[\boldsymbol{\alpha}]^{\mathbf{2 0}}{ }_{\mathbf{D}}+77.24\left(\mathrm{c} \mathrm{0.87,} \mathrm{CHCl}_{3}\right){ }^{\mathbf{1}} \mathbf{H} \mathbf{N M R}\left(500 \mathrm{MHz}, \mathrm{C}_{6} \mathrm{D}_{6}\right) \delta 7.26-7.25(\mathrm{~m}, 2$ H), 7.19-7.15 (m, $2 \mathrm{H}), 7.12-7.06(\mathrm{~m}, 1 \mathrm{H}), 5.56$ (ddt, $J=10.0,3.8$ and 2.0 Hz, $1 \mathrm{H}$ ), 5.13 (ddt, $J=10.0,3.8$ and $1.7 \mathrm{~Hz}, 1 \mathrm{H}), 4.57-4.55(\mathrm{~m}, 1 \mathrm{H}), 4.36$ and $4.32(\mathrm{AB}$ system, $J=12.1 \mathrm{~Hz}, 2 \mathrm{H})$, 4.05-3.99 (m, 2 H), 3.56-3.52 (m, 2 H), 2.57 (ddd, $J=21.6,3.7$ and 2.0 Hz, $1 \mathrm{H}), 2.43$ (ddt, $J=$ 
21.6, 3.6 and $2.0 \mathrm{~Hz}, 1 \mathrm{H}), 1.36(\mathrm{~s}, 3 \mathrm{H}), 1.29$ (s, $3 \mathrm{H}) ;{ }^{13} \mathrm{C} \mathbf{N M R}\left(125 \mathrm{MHz}, \mathrm{C}_{6} \mathrm{D}_{6}\right) \delta 166.1$, 138.2, 128.1 (2 C), 127.7 (2 C), 127.4, 123.1, 122.8, 109.6, 79.2, 79.1, 78.2, 73.1, 70.2, 29.7, 26.8, 26.6; IR $v_{\max } / \mathrm{cm}^{-1} 2986,2870,1743,1454,1371,1218,1089,857,739,699 ; \mathbf{M S} \mathrm{m} / \mathrm{z}(\mathrm{EI})$ $m / z 318 \mathrm{M}^{+}$; HRMS (EI): $m / z$ calcd for $\mathrm{C}_{18} \mathrm{H}_{22} \mathrm{O}_{5}: 318.1467$, found 318.1476. $\mathbf{C}_{18} \mathbf{H}_{22} \mathbf{O}_{5}(\mathbf{M}=$ $318.36 \mathrm{~g} / \mathrm{mol}$ ) calcd C: 67.91, H: 6.97 found C: 67.78, H: 6.79.

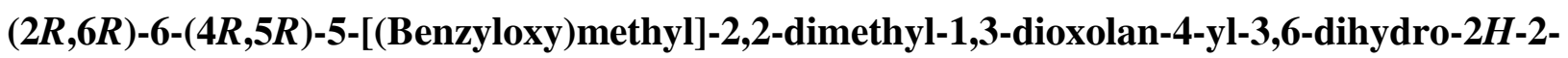
pyranyl acetate (12)

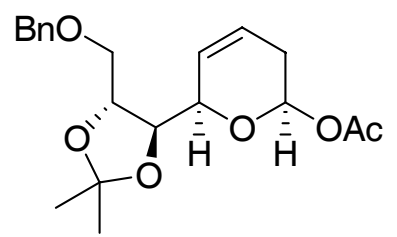

A solution of DIBAL (1 $\mathrm{M}$ in toluene, $33.4 \mathrm{~mL}, 31.8 \mathrm{mmol}$ ) was added dropwise over a period of $40 \mathrm{~min}$ to a solution of lactone $11(5.06 \mathrm{~g}, 15.90 \mathrm{mmol})$ in DCM $(200 \mathrm{~mL})$ at $-78^{\circ} \mathrm{C}$. After stirring at $-78{ }^{\circ} \mathrm{C}$ for $1 \mathrm{~h}$, pyridine (4.11 mL, $\left.50.89 \mathrm{mmol}\right)$, DMAP (4.08 g, $\left.33.39 \mathrm{mmol}\right)$ and acetic anhydride $(9.6 \mathrm{~mL}, 101.78 \mathrm{mmol})$ were sequentially added. The reaction mixture was then slowly warmed up to rt over a period of $14 \mathrm{~h}$. The reaction mixture was then quenched by addition of sat. aq. $\mathrm{NH}_{4} \mathrm{Cl}(100 \mathrm{~mL})$ and sat. aq. $\mathrm{Na} / \mathrm{K}$ tartrate $(100 \mathrm{~mL})$ and stirred for $1.5 \mathrm{~h}$ at rt. The organic phase was separated and washed with cold $1 \mathrm{~N} \mathrm{NaHSO}_{4}(100 \mathrm{~mL})$ and cold sat. aq. $\mathrm{NaHCO}_{3}(100 \mathrm{~mL})$. The organic phase was separated, dried over $\mathrm{Na}_{2} \mathrm{SO}_{4}$, filtered and evaporated under reduced pressure to provide the desired acetoxy ether $12(5.64 \mathrm{~g}, 98 \%)$ as a colorless oil in a 98:2 diastereomeric ratio. This oil was normally used in the following synthetic step without further purification. $\mathrm{R}_{\mathrm{f}}=0.45$ (eluent hexanes/ether $2: 1$ ); $[\boldsymbol{\alpha}]^{\mathbf{2 0}} \mathrm{D}+87.91$ (c 1.15, $\left.\mathrm{CHCl}_{3}\right) ;{ }^{1} \mathbf{H}$ NMR $\left(400 \mathrm{MHz}, \mathrm{C}_{6} \mathrm{D}_{6}\right) \delta$ 7.30-7.28 (m, $\left.2 \mathrm{H}\right), 7.17-7.14(\mathrm{~m}, 2 \mathrm{H}), 7.09-7.05$ (m, 1 $\mathrm{H}), 6.08(\mathrm{dd}, J=5.3$ and $3.9 \mathrm{~Hz}, 1 \mathrm{H}), 5.97(\mathrm{ddd}, J=10.4,3.8$ and $1.9 \mathrm{~Hz}, 1 \mathrm{H}), 5.45(\mathrm{ddt}, J=$ 10.4, 4.0 and $2.4 \mathrm{~Hz}, 1 \mathrm{H}$ ), 4.47 and 4.43 (AB system, $J=12.1 \mathrm{~Hz}, 2 \mathrm{H}), 4.31-4.26$ (m, $2 \mathrm{H}$ ), $4.12(\mathrm{dd}, J=8.2$ and $7.5 \mathrm{~Hz}, 1 \mathrm{H}), 3.73(\mathrm{dd}, J=10.9$ and $2.7 \mathrm{~Hz}, 1 \mathrm{H}), 3.63(\mathrm{dd}, J=10.9$ and $4.5 \mathrm{~Hz}, 1 \mathrm{H}), 2.02-1.86(\mathrm{~m}, 1 \mathrm{H}), 1.63(\mathrm{~s}, 3 \mathrm{H}), 1.45(\mathrm{~s}, 3 \mathrm{H}), 1.35(\mathrm{~s}, 3 \mathrm{H}) ;{ }^{13} \mathbf{C}$ NMR (100 $\left.\mathrm{MHz}, \mathrm{C}_{6} \mathrm{D}_{6}\right) \delta 169.3,139.6,129.0$ (2 C), 128.0 (2 C), 127.8, 127.4, 122.7, 110.0, 90.9, 81.1, 78.6, 76.6, 73.9, 71.1, 29.6, 27.9, 27.8, 21.2; IR $v_{\max } / \mathrm{cm}^{-1} 2987,2943,1746,1370,1239,1048$, 932, 857, 737, 699; MS m/z (ES) $m / z, 385(\mathrm{M}+\mathrm{Na})^{+}$; HRMS (Es): $m / z$ calcd for $\mathrm{C}_{20} \mathrm{H}_{26} \mathrm{O}_{6} \mathrm{Na}$ : 385.1627 , found 385.1611 . 
$(2 R, 4 R, 5 R, 6 R)-6-(4 R, 5 R)-5-[(B e n z y l o x y) m e t h y l]-2,2-d i m e t h y l-1,3-d i o x o l a n-4-y l-4,5-$ dihydroxytetrahydro-2H-2-pyranyl acetate (13)

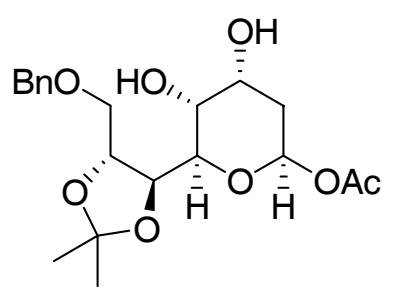

A sample of $N$-methylmorpholine- $N$-oxide $(2.16 \mathrm{~g}, 18.4 \mathrm{mmol})$ and $\mathrm{OsO}_{4}(2.5 \%$ in $t$ $\mathrm{BuOH}, 2.60 \mathrm{~g}, 0.25 \mathrm{mmol})$ were sequentially added to a solution of acetoxy ether 12 (2.10 g, 5.8 $\mathrm{mmol})$ in acetone $/ \mathrm{H}_{2} \mathrm{O}(8: 1)(50 \mathrm{~mL})$ at $\mathrm{rt}$. After stirring at $\mathrm{rt}$ for $16 \mathrm{~h}$, the reaction was quenched by addition of sat. aq. $\mathrm{Na}_{2} \mathrm{SO}_{3}(100 \mathrm{~mL})$ and ether $(200 \mathrm{~mL})$. The organic phase was separated and the aqueous extracted with ether $(2 \times 50 \mathrm{~mL})$. The combined organic extracts were dried over $\mathrm{MgSO}_{4}$, filtered and evaporated under reduced pressure to give a residue. This crude material was purified by column chromatography (hexanes/ether 1:4) to afford diol $\mathbf{1 3}$ (2.16 g, 94\%) as a colorless oil in a 96:4 diastereomeric ratio: $\mathrm{R}_{\mathrm{f}}=0.45$ (eluent hexanes/ether 2:1); $[\boldsymbol{\alpha}]^{\mathbf{2 0}}{ }_{\mathrm{D}}$ +32.74 (c 0.53, $\left.\mathrm{CHCl}_{3}\right) ;{ }^{1} \mathbf{H}$ NMR (400 MHz, $\left.\mathrm{C}_{6} \mathrm{D}_{6}\right) \delta 7.30-7.28(\mathrm{~m}, 2 \mathrm{H}), 7.17-7.14(\mathrm{~m}, 2 \mathrm{H})$, 7.09-7.05 (m, $1 \mathrm{H}), 6.24$ (dd, $J=10.0$ and $2.2 \mathrm{~Hz}, 1 \mathrm{H}$ ), 4.48 and 4.41 (AB system, $J=12.2 \mathrm{~Hz}$, $2 \mathrm{H}), 4.29(\mathrm{ddd}, J=7.4,4.6$ and $2.6 \mathrm{~Hz}, 1 \mathrm{H}), 4.10(\mathrm{t}, J=7.8 \mathrm{~Hz}, 1 \mathrm{H}), 4.01(\mathrm{dd}, J=6.2$ and 3.1 $\mathrm{Hz}, 1 \mathrm{H}), 3.90(\mathrm{dd}, J=8.2$ and $9.1 \mathrm{~Hz}, 1 \mathrm{H}), 3.82(\mathrm{~s}, 1 \mathrm{H}), 3.71(\mathrm{dd}, J=11.0$ and $2.6 \mathrm{~Hz}, 1 \mathrm{H})$, $3.62(\mathrm{dd}, J=11.0$ and $4.8 \mathrm{~Hz}, 1 \mathrm{H}), 3.51(\mathrm{ddd}, J=9.3,2.9$ and $1.0 \mathrm{~Hz}, 1 \mathrm{H}), 3.09(\mathrm{~s}, 1 \mathrm{H}), 2.06$ (ddd, $J=13.5,3.7$ and $2.5 \mathrm{~Hz}, 1 \mathrm{H}), 1.61-1.56(\mathrm{~m}, 1 \mathrm{H}), 1.58(\mathrm{~s}, 3 \mathrm{H}), 1.33(\mathrm{~s}, 3 \mathrm{H}), 1.21(\mathrm{~s}, 3$ $\mathrm{H}) ;{ }^{13} \mathrm{C}$ NMR (100 MHz, $\left.\mathrm{C}_{6} \mathrm{D}_{6}\right) \delta$ 169.1, 139.4, 128.9 (2 C), 128.0 (2 C), 127.9, 110.8, 91.2, 81.6, 79.0, 74.8, 73.9, 71.9, 70.9, 67.6, 35.7, 27.5, 27.1, 20.8; IR $v_{\max } / \mathrm{cm}^{-1} 3492,2985,2932$, 1754, 1453, 1368, 1214, 1045, 855, 738, 699; MS m/z (EI) $\mathrm{m} / \mathrm{z} 381\left(\mathrm{M}-\mathrm{CH}_{3}\right)^{+}$; HRMS (EI): $m / z$ calcd for $\mathrm{C}_{19} \mathrm{H}_{25} \mathrm{O}_{8}: 381.1549$, found 381.1559 . $\mathbf{C}_{20} \mathbf{H}_{28} \mathbf{O}_{\mathbf{8}}(\mathrm{M}=369.43 \mathrm{~g} / \mathrm{mol})$ calcd $\mathrm{C}$ : 60.59, H: 7.12 found C: $60.38, \mathrm{H}: 6.92$. 
Stereochemical assignment of diol 13: spin-coupling constants ${ }^{3} J_{\mathrm{H}-\mathrm{H}}$ and NOE observed

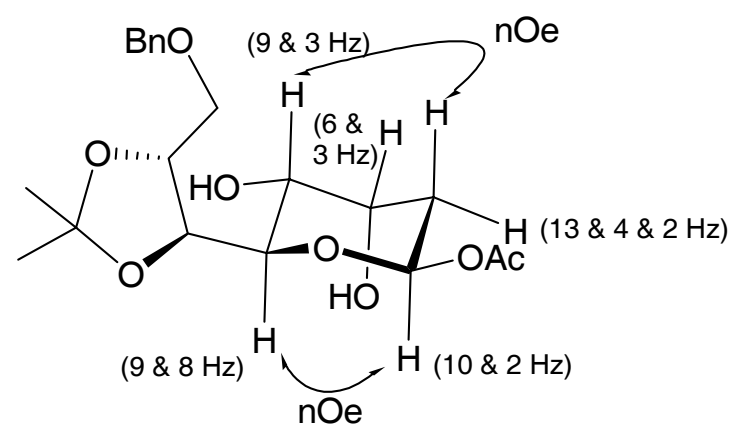

$(3 \mathrm{a} S, 4 R, 6 S, 7 \mathrm{a} R)-$ and $(3 \mathrm{a} S, 4 R, 6 R, 7 \mathrm{a} R)-4-(4 R, 5 R)-5$-[(Benzyloxy)methyl]-2,2-dimethyl-1,3dioxolan-4-yl-6-methoxy-2,2-dimethylperhydro[1,3]dioxolo[4,5-c]pyran (32eq and 32ax)
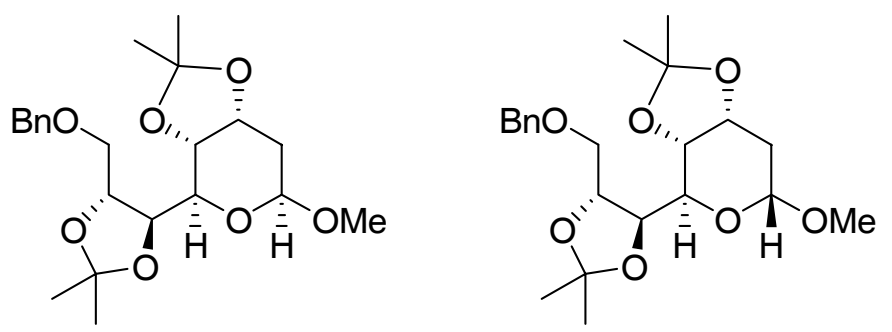

2,2-dimethoxypropane (1.06 g, $10.2 \mathrm{mmol})$ and $p$-toluenesulfonic acid (54 mg, 0.284 $\mathrm{mmol})$ both were added to a solution of diol $13(2.01 \mathrm{~g}, 5.1 \mathrm{mmol})$ in benzene $(25 \mathrm{~mL})$ at $\mathrm{rt}$. The reaction mixture was stirred at $\mathrm{rt}$ overnight. Sat. aq. $\mathrm{NaHCO}_{3}(20 \mathrm{~mL})$ and ether $(50 \mathrm{~mL})$ were sequentially added. The organic phase was separated and the aqueous was extracted with ether (30 mL). The combined organic extracts were dried over $\mathrm{MgSO}_{4}$, filtered and evaporated under reduced pressure to give the crude oil. This crude material was purified by column chromatography (hexanes/ether 1:1) to afford acetals 32eq (885 mg, 43\%) and 32ax (880 mg, $42 \%)$.

32eq; colorless oil; $\mathrm{R}_{f}=0.6$ (eluent hexanes/ether 1:1); $[\boldsymbol{\alpha}]^{\mathbf{2 0}}{ }_{\mathbf{D}}+7.35\left(\mathrm{c} 0.41, \mathrm{CHCl}_{3}\right) ;{ }^{\mathbf{1}} \mathbf{H}$ NMR (500 MHz, C $\left.6 \mathrm{D}_{6}\right) \delta$ 7.29-7.27 (m, $\left.2 \mathrm{H}\right), 7.17-7.14$ (m, $\left.2 \mathrm{H}\right), 7.10-7.07$ (m, $\left.1 \mathrm{H}\right), 4.71$ (dt, $J=7.8$ and $5.0 \mathrm{~Hz}, 1 \mathrm{H}), 4.64(\mathrm{dd}, J=8.7$ and $2.4 \mathrm{~Hz}, 1 \mathrm{H}) 4.44(\mathrm{dd}, J=7.8$ and $2.6 \mathrm{~Hz}, 1 \mathrm{H})$, $4.37(\mathrm{~m}, 2 \mathrm{H}), 4.22(\mathrm{dd}, J=9.3$ and $5.4 \mathrm{~Hz}, 1 \mathrm{H}), 4.05(\mathrm{td}, J=5.0$ and $4.5 \mathrm{~Hz}, 1 \mathrm{H}), 3.84(\mathrm{dd}, J=$ 9.2 and $2.6 \mathrm{~Hz}, 1 \mathrm{H}), 3.67(\mathrm{dd}, J=10.0$ and $4.8 \mathrm{~Hz}, 1 \mathrm{H}), 3.59(\mathrm{dd}, J=10.0$ and $5.3 \mathrm{~Hz}, 1 \mathrm{H})$, $3.29(\mathrm{~s}, 3 \mathrm{H}), 2.14(\mathrm{dt}, J=14.4$ and $2.6 \mathrm{~Hz}, 1 \mathrm{H}), 1.80(\mathrm{ddd}, J=14.4,8.8$ and $4.8 \mathrm{~Hz}, 1 \mathrm{H}), 1.54$ $(\mathrm{s}, 3 \mathrm{H}), 1.47$ (s, $3 \mathrm{H}), 1.41(\mathrm{~s}, 3 \mathrm{H}), 1.19(\mathrm{~s}, 3 \mathrm{H}) ;{ }^{13} \mathrm{C}$ NMR $\left(100 \mathrm{MHz}, \mathrm{C}_{6} \mathrm{D}_{6}\right) \delta 138.9,128.6(2$ C), 128.4 (2 C), 127.7, 109.6, 109.0, 100.2, 80.4, 76.2, 75.4, 73.6, 73.2, 71.8, 71.4, 56.1, 33.3, 28.4, 27.8, 27.3, 26.0; IR $v_{\max } / \mathrm{cm}^{-1} 2986,2934,1454,1372,1244,1161,1082,850$, 699; MS 
$\mathrm{m} / \mathrm{z}(\mathrm{EI}) 431(\mathrm{M}+\mathrm{Na})^{+}$; HRMS (EI): $\mathrm{m} / z$ calcd for $\mathrm{C}_{21} \mathrm{H}_{29} \mathrm{O}_{7}\left(\mathrm{M}-\mathrm{CH}_{3}\right)^{+}:$393.1913, found 393.1907.

32ax; colorless oil; $\mathrm{R}_{f}=0.5$ (eluent hexanes/ether 1:1); $[\mathbf{\alpha}]^{\mathbf{2 0}}{ }_{\mathbf{D}}-54.5\left(\mathrm{c} 0.33, \mathrm{CHCl}_{3}\right) ;{ }^{\mathbf{1}} \mathbf{H}$ NMR (500 MHz, $\left.\mathrm{C}_{6} \mathrm{D}_{6}\right) \delta$ 7.29-7.28 (m, $\left.2 \mathrm{H}\right), 7.17-7.15$ (m, $\left.2 \mathrm{H}\right), 7.10-7.07$ (m, $\left.1 \mathrm{H}\right), 4.67$ (dt, $J=8.9$ and $4.9 \mathrm{~Hz}, 1 \mathrm{H}), 4.48-4.41(\mathrm{~m}, 2 \mathrm{H}), 4.38(\mathrm{~m}, 2 \mathrm{H}), 4.37(\mathrm{dd}, J=8.9$ and $6.3 \mathrm{~Hz}, 1 \mathrm{H})$, $4.19(\mathrm{dd}, J=9.1$ and $2.8 \mathrm{~Hz}, 1 \mathrm{H}), 3.96(\mathrm{q}, J=6.3 \mathrm{~Hz}, 1 \mathrm{H}), 3.66(\mathrm{dd}, J=10.1$ and $4.8 \mathrm{~Hz}, 1 \mathrm{H})$, $3.62(\mathrm{dd}, J=10.1$ and $5.1 \mathrm{~Hz}, 1 \mathrm{H}), 3.19(\mathrm{~s}, 3 \mathrm{H}), 1.98(\mathrm{ddd}, J=10.1,6.8$ and $4.9 \mathrm{~Hz}, 1 \mathrm{H}), 1.85$ $(\mathrm{dt}, J=14.6$ and $5.6 \mathrm{~Hz}, 1 \mathrm{H}), 1.52(\mathrm{~s}, 3 \mathrm{H}), 1.46(\mathrm{~s}, 3 \mathrm{H}), 1.43(\mathrm{~s}, 3 \mathrm{H}), 1.22(\mathrm{~s}, 3 \mathrm{H}) ;{ }^{13} \mathbf{C} \mathbf{~ N M R}$ (100 MHz, $\left.\mathrm{C}_{6} \mathrm{D}_{6}\right) \delta 138.9,128.6$ (2 C), 128.4 (2 C), 127.7, 109.6, 109.4, 97.7, 80.4, 76.6, 73.6, 73.3, 71.9, 71.5, 69.0, 54.9, 32.7, 28.3, 27.7, 27.2, 25.8; IR $v_{\max } / \mathrm{cm}^{-1} 2986,2934,1454,1372$, 1244, 1161, 1082, 850, 699; MS m/z (EI) $431(\mathrm{M}+\mathrm{Na})^{+}$; HRMS (EI): $m / z$ calcd for $\mathrm{C}_{21} \mathrm{H}_{29} \mathrm{O}_{7}$ $\left(\mathrm{M}-\mathrm{CH}_{3}\right)^{+}:$393.1913, found 393.1907.

$(4 R, 5 R)-5-[(3 \mathrm{a} S, 4 R, 6 S, 7 \mathrm{a} R)-\quad$ and $\quad(4 R, 5 R)-5-[(3 \mathrm{a} S, 4 R, 6 R, 7 \mathrm{a} R)-6-\mathrm{Methoxy}-2,2-$ dimethylperhydro[1,3]dioxolo[4,5-c]pyran-4-yl]-2,2-dimethyl-1,3-dioxolan-4-ylmethanol (14eq and 14ax)
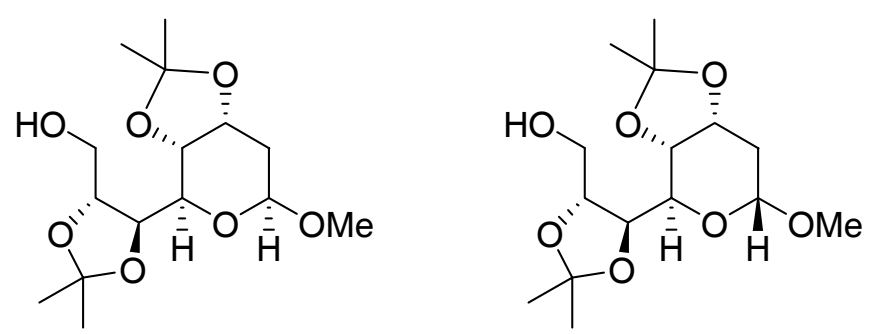

A solution of a 1:1 mixture of benzyl ethers 32ax and 32eq $(4.28 \mathrm{~g}, 10.5 \mathrm{mmol})$ in methanol $(20 \mathrm{~mL})$ was added to a flask containing palladium $10 \%$ on activated carbon $(550 \mathrm{mg})$ under argon. The resultant heterogeneous mixture was purged with $\mathrm{H}_{2}$ and stirred under $\mathrm{H}_{2}(c a$. $1 \mathrm{~atm})$ at $\mathrm{rt}$ for $40 \mathrm{~h}$. The reaction mixture was then purged with argon, diluted with DCM (80 $\mathrm{mL}$ ) and filtered through a celite $®$ plug. The filtrated was evaporated under reduced pressure to give the crude material. The crude oil was purified by column chromatography (hexanes/ether 1:1) to provide alcohols $\mathbf{1 4} \mathbf{e q}$ and $\mathbf{1 4 a x}(3.27 \mathrm{~g}, 98 \%)$ as a $1: 1$ mixture of isomers.

14eq; colorless oil characterized from an enriched mixture of both diastereomers; $\mathrm{R}_{f}=$ 0.18 (eluent hexanes/ether 1:1); ${ }^{1} \mathbf{H}$ NMR $\left(500 \mathrm{MHz} \mathrm{CDCl}_{3}\right) \delta 4.67$ (dd, $J=9.0$ and $2.4 \mathrm{~Hz}, 1$ H), $4.46(\mathrm{td}, J=4.9$ and $2.7 \mathrm{~Hz}, 1 \mathrm{H}) 4.32(\mathrm{ddd}, J=8.2,4.9$ and $3.9 \mathrm{~Hz}, 1 \mathrm{H}), 4.15-4.11(\mathrm{~m}, 2$ H), 3.86 (ddd, $J=11.9,5.1$ and $3.7 \mathrm{~Hz}, 1 \mathrm{H}$ ), 3.70 (ddd, $J=11.9,7.6$ and $4.5 \mathrm{~Hz}, 1 \mathrm{H}), 3.59$ (dd, 
$J=9.0$ and $4.1 \mathrm{~Hz}, 1 \mathrm{H}), 3.48(\mathrm{~s}, 3 \mathrm{H}), 2.25(\mathrm{dt}, J=14.8$ and $2.5 \mathrm{~Hz}, 1 \mathrm{H}), 2.03(\mathrm{dd}, J=7.6$ and $5.3 \mathrm{~Hz}, 1 \mathrm{H}), 1.92$ (ddd, $J=14.7,9.0$ and $4.5 \mathrm{~Hz}, 1 \mathrm{H}), 1.48(\mathrm{~s}, 3 \mathrm{H}), 1.47$ (s, $3 \mathrm{H}), 1.47$ (s, $3 \mathrm{H})$, 1.36 (s, $3 \mathrm{H}) ;{ }^{13} \mathbf{C}$ NMR $\left(125 \mathrm{MHz}, \mathrm{CDCl}_{3}\right) \delta$ 109.5, 109.1, 99.7, 77.8, 77.5, 74.8, 72.9, 71.1, $62.5,56.5,32.6,28.1,27.2,26.8,25.8$.

14ax; white solid; $\mathrm{R}_{f}=0.15$ (eluent hexanes/ether 1:1); m.p. $84-86^{\circ} \mathrm{C}$; $[\boldsymbol{\alpha}]^{\mathbf{2 0}}{ }_{\mathrm{D}}-70.6$ (c 0.5, $\left.\mathrm{CH}_{2} \mathrm{Cl}_{2}\right) ;{ }^{1} \mathbf{H}$ NMR $\left(500 \mathrm{MHz}, \mathrm{CDCl}_{3}\right) \delta 4.78(\mathrm{dd}, J=5.1$ and $4.4 \mathrm{~Hz}, 1 \mathrm{H}), 4.33-4.27$ (m, 3 $\mathrm{H}), 4.17(\mathrm{dd}, J=8.8$ and $3.2 \mathrm{~Hz}, 1 \mathrm{H}), 3.87(\mathrm{dd}, J=12.0,5.1$ and $3.7 \mathrm{~Hz}, 1 \mathrm{H}), 3.82(\mathrm{dd}, J=8.1$ and $2.9 \mathrm{~Hz}, 1 \mathrm{H}), 3.70(\mathrm{t}, J=12.0,7.8$ and $4.4 \mathrm{~Hz}, 1 \mathrm{H}), 3.37(\mathrm{~s}, 3 \mathrm{H}), 2.19(\mathrm{dt}, J=11.0$ and 5.4 $\mathrm{Hz}, 1 \mathrm{H}), 2.04-1.97$ (m, 2 H), 1.63 (s, $3 \mathrm{H}), 1.48$ (s, $3 \mathrm{H}), 1.47$ (s, $3 \mathrm{H}), 1.35$ (s, $3 \mathrm{H})$; ${ }^{13} \mathbf{C}$ NMR $\left(125 \mathrm{MHz}, \mathrm{CDCl}_{3}\right) \delta 109.5,109.4,97.3,77.8,77.2$, 77.1, 70.9, 67.5, 62.0, 55.5, 31.7, 28.1, 27.2, 26.7, 25.7; IR $v_{\max } / \mathrm{cm}^{-1} 3480,2987,2935,1372,1245,1218,1165,1124,1046 ;$ MS m/z (EI) $303\left(\mathrm{M}-\mathrm{CH}_{3}\right)^{+}$; HRMS (EI): $\mathrm{m} / z$ calcd for $\mathrm{C}_{14} \mathrm{H}_{23} \mathrm{O}_{7}: 303.1437$, found 303.1443 .

$(4 R, 5 R)-5-[(3 \mathrm{a} S, 4 R, 6 S, 7 \mathrm{a} R)-\quad$ and $\quad(4 R, 5 R)-5-[(3 \mathrm{a} S, 4 R, 6 R, 7 \mathrm{a} R)-6-\mathrm{Methoxy}-2,2-$ dimethylperhydro[1,3]dioxolo[4,5-c]pyran-4-yl]-2,2-dimethyl-1,3-dioxolan-4ylmethoxy)(tert-butyl)dimethylsilane (33eq and 33ax)
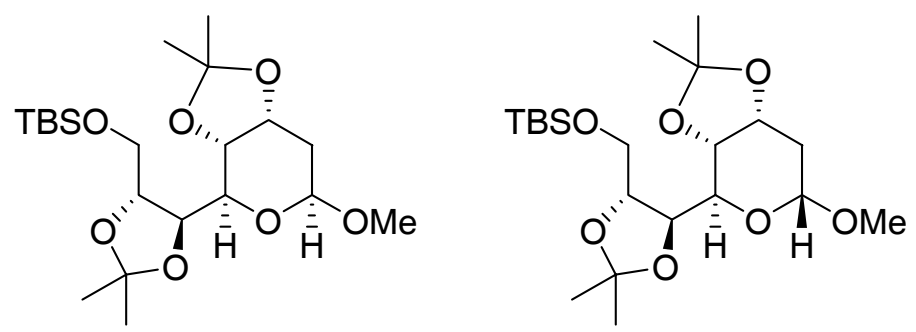

A solution of a 1:1 mixture of alcohols $14(1.33 \mathrm{~g}, 4.18 \mathrm{mmol}), \mathrm{TBSCl}(1.26 \mathrm{~g}, 8.36$ mmol), imidazole (1.14 g, $16.72 \mathrm{mmol})$ and DMAP (26 mg, $0.209 \mathrm{mmol})$ in anhydrous DMF $(10 \mathrm{~mL})$ was stirred at $\mathrm{rt}$ for $14 \mathrm{~h}$. Ether $(200 \mathrm{~mL})$ and water $(200 \mathrm{~mL})$ were added. The organic phase was separated and the aqueous was extracted with ether $(100 \mathrm{~mL})$. The combined organic phases were dried over $\mathrm{MgSO}_{4}$, filtered and evaporated under reduced pressure to give the crude oil. This oil was purified by column chromatography (hexanes/ether 2:1) to afford alcohols 33eq and 33ax $(1.6 \mathrm{~g}, 90 \%)$ as a 1:1 mixture of isomers.

33eq; colorless oil; $\mathrm{R}_{f}=0.4$ (eluent hexanes/ether 2:1); $[\boldsymbol{\alpha}]^{\mathbf{2 0}}{ }_{\mathbf{D}}+9.9\left(\mathrm{c} 1.55, \mathrm{CH}_{2} \mathrm{Cl}_{2}\right) ;{ }^{\mathbf{1}} \mathbf{H}$ NMR $\left(400 \mathrm{MHz}, \mathrm{CDCl}_{3}\right) \delta 4.66(\mathrm{dd}, J=8.9$ and $2.3 \mathrm{~Hz}, 1 \mathrm{H}), 4.44(\mathrm{td}, J=4.7$ and $2.5 \mathrm{~Hz}, 1 \mathrm{H})$, $4.34(\mathrm{ddd}, J=7.8,6.1$ and $4.3 \mathrm{~Hz}, 1 \mathrm{H}), 4.26(\mathrm{dd}, J=9.2$ and $5.3 \mathrm{~Hz}, 1 \mathrm{H}), 4.19(\mathrm{dd}, J=7.8$ and $2.5 \mathrm{~Hz}, 1 \mathrm{H}), 3.83(\mathrm{dd}, J=10.6$ and $4.3 \mathrm{~Hz}, 1 \mathrm{H}), 3.73(\mathrm{ddd}, J=10.6$ and $6.1 \mathrm{~Hz}, 1 \mathrm{H}), 3.65$ (dd, 
$J=9.4$ and $2.5 \mathrm{~Hz}, 1 \mathrm{H}), 3.48(\mathrm{~s}, 3 \mathrm{H}), 2.24(\mathrm{dt}, J=14.7$ and $2.4 \mathrm{~Hz}, 1 \mathrm{H}), 1.91$ (ddd, $J=14.7$, 9.0 and $4.7 \mathrm{~Hz}, 1 \mathrm{H}), 1.49$ (s, $3 \mathrm{H}), 1.46$ (s, $3 \mathrm{H}), 1.43$ (s, $3 \mathrm{H}), 1.34$ (s, $3 \mathrm{H}), 0.91(\mathrm{~s}, 9 \mathrm{H}), 0.09$ $(\mathrm{s}, 3 \mathrm{H}), 0.08(\mathrm{~s}, 3 \mathrm{H}) ;{ }^{13} \mathbf{C}$ NMR $\left(125 \mathrm{MHz}, \mathrm{CDCl}_{3}\right) \delta 109.3,108.9,99.7,79.8,76.5,74.7,72.9$, 70.4, 64.4, 56.3, 32.7, 28.1, 27.2, 26.8, 25.8, 25.8 (3 C), 18.3, -5.5, -5.5; IR $v_{\max } / \mathrm{cm}^{-1} 2987$, 2955, 2934, 2886, 2859, 1463, 1372, 1249, 1215, 1148, 1082, 840, 779; MS m/z (CI) 417 (M $\left.\mathrm{CH}_{3}\right)^{+}$; HRMS (CI): $\mathrm{m} / z$ calcd for $\mathrm{C}_{20} \mathrm{H}_{37} \mathrm{O}_{7} \mathrm{Si}: 417.2308$, found 417.2308.

33ax; colorless oil characterized from an enriched mixture of both diastereomers; $\mathrm{R}_{f}=$ 0.3 (eluent hexanes/ether 2:1); ${ }^{1} \mathbf{H}$ NMR $\left(500 \mathrm{MHz} \mathrm{CDCl}_{3}\right) \delta 4.78(\mathrm{t}, J=4.9 \mathrm{~Hz}, 1 \mathrm{H}), 4.42(\mathrm{dd}$, $J=9.0$ and $6.1 \mathrm{~Hz}, 1 \mathrm{H}), 4.33-4.25(\mathrm{~m}, 2 \mathrm{H}), 4.23(\mathrm{dd}, J=8.6$ and $2.2 \mathrm{~Hz}, 1 \mathrm{H}), 3.89(\mathrm{dd}, J=$ 8.8 and $2.0 \mathrm{~Hz}, 1 \mathrm{H}), 3.83(\mathrm{dd}, J=10.5$ and $3.9 \mathrm{~Hz}, 1 \mathrm{H}), 3.75(\mathrm{dd}, J=10.5$ and $5.6 \mathrm{~Hz}, 1 \mathrm{H})$, $3.38(\mathrm{~s}, 3 \mathrm{H}), 2.19(\mathrm{dt}, J=14.7$ and $5.4 \mathrm{~Hz}, 1 \mathrm{H}), 1.96(\mathrm{ddd}, J=14.7,6.6$ and $4.6 \mathrm{~Hz}, 1 \mathrm{H}), 1.48$ (s, 3 H), 1.47 (s, 3 H), 1.45 (s, 3 H), 1.34 (s, 3 H), 0.91 (s, 9 H), 0.08 (s, 3 H), 0.09 (s, 3 H).

$(4 R, 5 S)-5-[(3 \mathrm{a} R, 4 R, 6 R, 7 \mathrm{a} R)-\quad$ and $\quad(4 R, 5 S)-5-[(3 \mathrm{a} R, 4 R, 6 S, 7 \mathrm{a} R)-2,2-\mathrm{Dimethyl-6-}$ (phenylsulfanyl)perhydro[1,3]dioxolo[4,5-c]pyran-4-yl]-2,2-dimethyl-1,3-dioxolan-4ylmethoxy)(tert-butyl)dimethylsilane (5eq and 5ax)
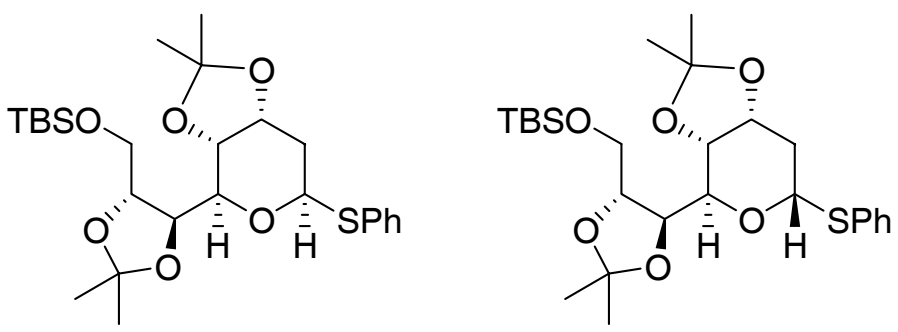

To a flask containing a 1:1 mixture of methyl acetals 33 (315 mg, $0.73 \mathrm{mmol}), n$ tetrabutylammonium iodide (269 $\mathrm{mg}, 0.73 \mathrm{mmol}), \mathrm{ZnI}_{2}(465 \mathrm{mg}, 1.46 \mathrm{mmol})$ and phenylthiotrimethylsilane $(414 \mu \mathrm{L}, 2.19 \mathrm{mmol})$ was added anhydrous 1,2-dichloroethane (5 $\mathrm{mL}$ ). The resultant mixture was heated at $85^{\circ} \mathrm{C}$ until no starting material was detected by ES analysis of aliquots (normally $40 \mathrm{~min}$ ). The reaction mixture was cooled to rt, diluted with DCM $(50 \mathrm{~mL})$ and treated with $0.5 \mathrm{~N} \mathrm{NaOH}(15 \mathrm{~mL})$. The organic phase was separated and washed with brine $(20 \mathrm{~mL})$. The organic phase was then dried over $\mathrm{MgSO}_{4}$, filtered and evaporated under reduced pressure to give a residue. The crude material was purified by column chromatography (hexanes/ether 4:1) to afford tetrahydropyrans 5 (260 $\mathrm{mg}, 70 \%$ ) as a 1:1 mixture of isomers. 
5eq; colorless oil; $\mathrm{R}_{f}=0.4$ (eluent hexanes/ether 4:1); $[\boldsymbol{\alpha}]^{\mathbf{2 0}}{ }_{\mathbf{D}}+31.94\left(\mathrm{c} 1.8, \mathrm{CH}_{2} \mathrm{Cl}_{2}\right) ;{ }^{1} \mathbf{H}$ NMR $\left(500 \mathrm{MHz}, \mathrm{CDCl}_{3}\right) \delta 7.55(\mathrm{~m}, 2 \mathrm{H}), 7.36-7.28(\mathrm{~m}, 3 \mathrm{H}), 5.10(\mathrm{dd}, J=12.0$ and $2.4 \mathrm{~Hz}, 1$ $\mathrm{H}), 4.46(\mathrm{td}, J=4.9$ and $1.7 \mathrm{~Hz}, 1 \mathrm{H}), 4.35-4.31(\mathrm{~m}, 2 \mathrm{H}), 4.22(\mathrm{dd}, J=7.8$ and $2.7 \mathrm{~Hz}, 1 \mathrm{H})$, $3.84(\mathrm{dd}, J=10.3$ and $3.9 \mathrm{~Hz}, 1 \mathrm{H}), 3.75(\mathrm{dd}, J=10.6$ and $6.1 \mathrm{~Hz}, 1 \mathrm{H}), 3.70(\mathrm{dd}, J=9.3$ and 2.7 $\mathrm{Hz}, 1 \mathrm{H}), 2.47(\mathrm{dt}, J=14.9$ and $2.2 \mathrm{~Hz}, 1 \mathrm{H}), 2.15(\mathrm{ddd}, J=14.7,12.0$ and $4.2 \mathrm{~Hz}, 1 \mathrm{H}), 1.54(\mathrm{~s}$, $3 \mathrm{H}), 1.47$ (s, $3 \mathrm{H}), 1.46$ (s, $3 \mathrm{H}), 1.40$ (s, $3 \mathrm{H}), 0.94$ (s, $9 \mathrm{H}), 0.11$ (s, $3 \mathrm{H}), 0.10$ (s, $3 \mathrm{H}) ;{ }^{13} \mathrm{C}$ NMR $\left(125 \mathrm{MHz}, \mathrm{CDCl}_{3}\right) \delta 134.3,131.1$ (2 C), 128.7 (2 C), 127.1, 109.3, 109.0, 81.2, 79.7, 77.8, 76.6, 72.5, 70.0, 64.2, 33.0, 28.2, 27.2, 26.8, 26.1, 25.8 (3C), 18.2, -5.4, -5.5; IR $v_{\max } / \mathrm{cm}^{-1}$ 2986, 2955, 2932, 2884, 2859, 1480, 1369, 1249, 1220, 1158, 1075, 840, 780; MS m/z (CI) 495 $\left(\mathrm{M}-\mathrm{CH}_{3}\right)^{+}$; HRMS (CI): $\mathrm{m} / z$ calcd for $\mathrm{C}_{25} \mathrm{H}_{39} \mathrm{O}_{6} \mathrm{SiS}: 495.2231$, found 495.2236.

5ax; colorless oil; $[\mathbf{\alpha}]^{\mathbf{2 0}}{ }_{\mathbf{D}}-19.18\left(\mathrm{c} 1, \mathrm{CH}_{2} \mathrm{Cl}_{2}\right) ; \mathrm{R}_{f}=0.4$ (eluent hexanes/ether 2:1); ${ }^{\mathbf{1}} \mathbf{H}$ NMR $\left(500 \mathrm{MHz}, \mathrm{CDCl}_{3}\right) \delta 7.49-7.47(\mathrm{~m}, 2 \mathrm{H}), 7.28-7.19(\mathrm{~m}, 3 \mathrm{H}), 5.57(\mathrm{dd}, J=6.6$ and 4.2 $\mathrm{Hz}, 1 \mathrm{H}), 4.47(\mathrm{dd}, J=8.8$ and $5.6 \mathrm{~Hz}, 1 \mathrm{H}), 4.37-4.33(\mathrm{~m}, 2 \mathrm{H}), 4.29$ (ddd, $J=8.8,5.1$ and 3.9, $1 \mathrm{H}), 4.21(\mathrm{dd}, J=8.3$ and $2.2 \mathrm{~Hz}, 1 \mathrm{H}), 3.78(\mathrm{dd}, J=10.8$ and $4.2 \mathrm{~Hz}, 1 \mathrm{H}), 3.75(\mathrm{dd}, J=10.8$ and $5.4 \mathrm{~Hz}, 1 \mathrm{H}), 2.51(\mathrm{ddd}, J=14.9,6.6$ and $4.4 \mathrm{~Hz}, 1 \mathrm{H}), 2.25$ (ddd, $J=14.9,9.0$ and $4.6 \mathrm{~Hz}$, $1 \mathrm{H}), 1.54$ (s, $3 \mathrm{H}), 1.45$ (s, $3 \mathrm{H}), 1.44(\mathrm{~s}, 3 \mathrm{H}), 1.37$ (s, $3 \mathrm{H}), 0.87$ (s, $9 \mathrm{H}), 0.05$ (s, $3 \mathrm{H}), 0.03$ (s, $3 \mathrm{H}) ;{ }^{13} \mathrm{C}$ NMR (100 MHz, $\left.\mathrm{C}_{6} \mathrm{D}_{6}\right) \delta$ 138.1, 131.5 (2 C), 129.5 (2 C), 127.4, 110.2, 109.9, 83.2, 80.4, 78.4, 72.2, 72.1, 70.3, 65.0, 33.7, 28.9, 28.1, 27.8, 26.7, 26.7 (3 C), 19.1, -4.6, -4.7; IR $v_{\max } / \mathrm{cm}^{-1} 2986,2954,28.58,1482,1369,1250,1219,1087,839,780 ;$ MS m/z (CI) 495 (M $\left.\mathrm{CH}_{3}\right)^{+}$; HRMS (CI): $\mathrm{m} / z$ calcd for $\mathrm{C}_{25} \mathrm{H}_{39} \mathrm{O}_{6} \mathrm{SiS}: 495.2231$, found 495.2236.

Stereochemical assignment of phenylthio acetal 5eq: spin-coupling constants ${ }^{3} J_{\mathrm{H}-\mathrm{H}}$ and NOE observed

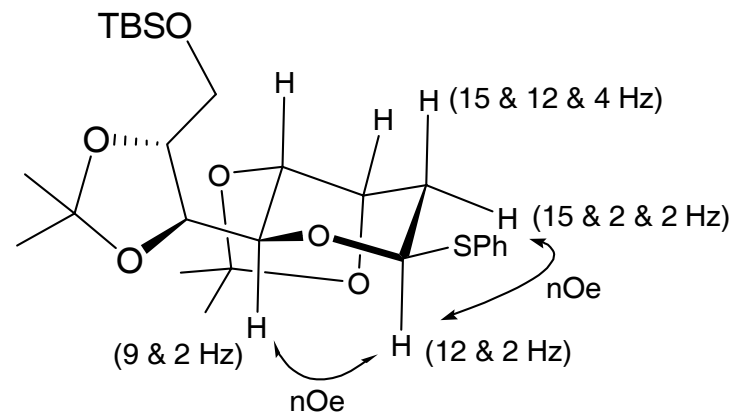




\section{Representative procedure for the reductive lithiation of phenylthio acetals and subsequent addition to electrophiles}

A 0.3 M solution of LiDBB in THF was freshly prepared by addition of anhydrous THF $(12.5 \mathrm{~mL})$ to a flask containing di-tert-butylbiphenyl $(1 \mathrm{~g}, 3.75 \mathrm{mmol})$ and lithium $(100 \mathrm{mg}, 14.4$ mmol) under argon at rt. The mixture was vigorously stirred with a Pyrex-coated stir-bar at rt until a permanent dark green color persisted. The green mixture was then stirred at $0{ }^{\circ} \mathrm{C}$ for $4 \mathrm{~h}$ and kept at that temperature before its use.

A solution of $n$-BuLi (2.4 M in hexanes, 2-3 drops) was added dropwise to a solution of a 1:1 mixture of phenylthio acetals $5(100 \mathrm{mg}, 0.197 \mathrm{mmol})$ and a couple of crystals of 1,10phenantroline in anhydrous THF $(2 \mathrm{~mL})$ at $-78{ }^{\circ} \mathrm{C}$ until an orange-red coloration just appeared. A freshly prepared $0.3 \mathrm{M}$ solution of LiDBB in THF was added dropwise via a gas tight syringe until a permanent dark green color persisted $(c a .1 .9 \mathrm{~mL}, 0.57 \mathrm{mmol})$. The resultant green solution was stirred at $-78{ }^{\circ} \mathrm{C}$ for 5 min. Freshly prepared epoxy aldehyde $6(66 \mu \mathrm{L}, 0.394$ mmol) was added to the reaction mixture. After addition of the electrophile, the resultant orange solution was stirred at $-78{ }^{\circ} \mathrm{C}$ for $30 \mathrm{~min}$. The reaction was quenched by sequential addition of methanol $(1 \mathrm{~mL})$ and sat. aq. $\mathrm{NH}_{4} \mathrm{Cl}(1 \mathrm{~mL})$ at $-78{ }^{\circ} \mathrm{C}$. The reaction mixture was allowed to warm to $\mathrm{rt}$ and diluted with ether $(10 \mathrm{~mL})$. The organic phase was separated and the aqueous was extracted with ether $(10 \mathrm{~mL})$. The combined organic extracts were dried over $\mathrm{MgSO}_{4}$, filtered and evaporated under reduced pressure to give a pale yellow residue. This material was purified by column chromatography (hexanes/ether 2:1) to afford alcohols $\mathbf{3}, \mathbf{3 b}, \mathbf{3 c}$ and $\mathbf{3 d}$ (89 $\mathrm{mg}, 82 \%)$ as a mixture of isomers (48:26:14:12). This mixture of isomers was partially separated by MPLC (gradient from hexanes/ether 2:1 to hexanes/ether 1:2). 


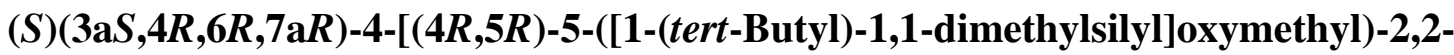
dimethyl-1,3-dioxolan-4-yl]-2,2-dimethylperhydro[1,3]dioxolo[4,5-c]pyran-6-yl)[(2S,3S)-3methyl-3-(4-methyl-3-pentenyl)oxiran-2-yl]methanol (3)

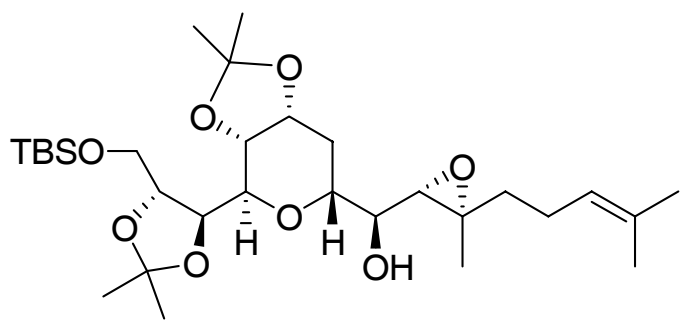

3; colorless oil; $\mathrm{R}_{f}=0.4$ (eluent hexanes/ether 2:1); $[\boldsymbol{\alpha}]^{\mathbf{2 0}}{ }_{\mathbf{D}}-4.18\left(\mathrm{c} 0.55, \mathrm{CH}_{2} \mathrm{Cl}_{2}\right)$; ${ }^{\mathbf{1}} \mathbf{H}$ NMR $\left(500 \mathrm{MHz} \mathrm{CDCl}_{3}\right) \delta 5.10(\mathrm{~m}, 1 \mathrm{H}), 4.39(\mathrm{dt}, J=8.6$ and $6.1 \mathrm{~Hz}, 1 \mathrm{H}), 4.28(\mathrm{t}, J=6.4 \mathrm{~Hz}$, $1 \mathrm{H}), 4.22(\mathrm{dd}, J=8.3$ and $2.4 \mathrm{~Hz}, 1 \mathrm{H}), 4.14(\mathrm{ddd}, J=8.1,5.4$ and $3.9 \mathrm{~Hz}, 1 \mathrm{H}), 4.01(\mathrm{dd}, J=$ 6.1 and $2.2 \mathrm{~Hz}, 1 \mathrm{H}), 3.89$ (ddd, $J=9.8,6.4$ and $4.9 \mathrm{~Hz}, 1 \mathrm{H}$ ), 3.84 (dd, $J=10.8$ and $4.2 \mathrm{~Hz}, 1$ H), $3.75(\mathrm{dd}, J=10.6$ and $5.6 \mathrm{~Hz}, 1 \mathrm{H}), 3.41(\mathrm{ddd}, J=8.3,6.4$ and $3.2 \mathrm{~Hz}, 1 \mathrm{H}), 2.75(\mathrm{~d}, J=3.5$ $\mathrm{Hz}, 1 \mathrm{H}), 2.73(\mathrm{~d}, J=8.4 \mathrm{~Hz}, 1 \mathrm{H}), 2.12(\mathrm{ddd}, J=14.2,10.6$ and $5.9 \mathrm{~Hz}, 1 \mathrm{H}), 1.87(\mathrm{dt}, J=14.2$ and $9.3 \mathrm{~Hz}, 1 \mathrm{H}), 1.68$ (s, $3 \mathrm{H}), 1.66-1.60$ (m, $2 \mathrm{H}), 1.61$ (s, $3 \mathrm{H}), 1.54-1.49$ (m, $2 \mathrm{H}), 1.49$ (s, 3 H), 1.44 (s, $3 \mathrm{H}), 1.42$ (s, $3 \mathrm{H}), 1.37$ (s, $3 \mathrm{H}), 1.35$ (s, $3 \mathrm{H}), 0.91(\mathrm{~s}, 9 \mathrm{H}), 0.09$ (s, $6 \mathrm{H}) ;{ }^{13} \mathbf{C} \mathbf{N M R}$ $\left(125 \mathrm{MHz} \mathrm{CDCl}_{3}\right) \delta 131.8,123.3,109.4,108.6,79.8,76.6,72.8,72.4,71.3,71.3,70.7,63.7$, $61.8,60.4,38.2,28.5,27.6,26.9,26.8,25.7,25.5$ (3 C), 23.5, 18.2, 17.5, 16.4, -5.5, -5.6; IR $v_{\max } / \mathrm{cm}^{-1} 3450,2931,2361,1560,1459,1380,1251,1068,838 ; \mathbf{M S} \mathrm{m} / \mathrm{z}(\mathrm{CI}) 555\left(\mathrm{M}-\mathrm{CH}_{3}\right)^{+}$; HRMS (CI): $m / z$ calcd for $\mathrm{C}_{29} \mathrm{H}_{41} \mathrm{O}_{8} \mathrm{Si}: 555.3344$, found 555.3353 .

Undesired isomer 3b; $\mathbf{R}_{f}=0.25$ (eluent hexanes/ether 2:1); ${ }^{1} \mathbf{H}$ NMR (500 MHz, $\mathrm{CDCl}_{3}$ ) (only representative signals given) $\delta 5.10(\mathrm{~m}, 1 \mathrm{H}), 4.44(\mathrm{~m}, 1 \mathrm{H}), 4.30(\mathrm{~m}, 1 \mathrm{H}), 4.25$ $(\mathrm{dd}, J=9.3$ and $5.1 \mathrm{~Hz}, 1 \mathrm{H}), 4.15(\mathrm{dd}, J=8.3$ and $2.2 \mathrm{~Hz}, 1 \mathrm{H}), 3.89(\mathrm{dt}, J=10.7$ and $2.7 \mathrm{~Hz}, 1$ H), $3.72(\mathrm{dd}, J=10.5$ and $5.6 \mathrm{~Hz}, 1 \mathrm{H}), 3.62(\mathrm{dd}, J=9.5$ and $2.4 \mathrm{~Hz}, 1 \mathrm{H}), 3.55(\mathrm{~m}, 1 \mathrm{H}), 2.83$ $(\mathrm{d}, J=8.6 \mathrm{~Hz}, 1 \mathrm{H}), 2.34(\mathrm{~d}, J=6.1 \mathrm{~Hz}, 1 \mathrm{H}) ; \mathbf{M S}(\mathrm{ES}) \mathrm{m} / \mathrm{z} 593(\mathrm{M}+\mathrm{Na})^{+}$.

Undesired isomer 3c; $\mathrm{R}_{f}=0.22$ (eluent hexanes/ether 2:1); ${ }^{1} \mathbf{H} \mathbf{N M R}\left(500 \mathrm{MHz}, \mathrm{CDCl}_{3}\right)$ (only representative signals given) $\delta 5.09(\mathrm{~m}, 1 \mathrm{H}), 4.38(\mathrm{dt}, J=10.5$ and $6.8,1 \mathrm{H}), 4.33(\mathrm{t}, J=$ $6.1 \mathrm{~Hz}, 1 \mathrm{H}), 4.19(\mathrm{dd}, J=8.6$ and $2.4 \mathrm{~Hz}, 1 \mathrm{H}), 4.01(\mathrm{dd}, J=5.9$ and $2.4 \mathrm{~Hz}, 1 \mathrm{H}), 2.89(\mathrm{~d}, J=$ $7.1 \mathrm{~Hz}, 1 \mathrm{H}), 2.25(\mathrm{~d}, J=6.1 \mathrm{~Hz}, 1 \mathrm{H}) ; \mathbf{M S}(\mathrm{ES}) \mathrm{m} / \mathrm{z} 593(\mathrm{M}+\mathrm{Na})^{+}$.

Undesired isomer 3d; $\mathrm{R}_{f}=0.15$ (eluent hexanes/ether 2:1); ${ }^{1} \mathbf{H}$ NMR (500 MHz, $\mathrm{CDCl}_{3}$ ) (only representative signals given) $\delta 5.08(\mathrm{~m}, 1 \mathrm{H}), 4.43(\mathrm{~m}, 1 \mathrm{H}), 4.30(\mathrm{~m}, 2 \mathrm{H}), 4.22$ 
$(\mathrm{dd}, J=8.1$ and $2.2 \mathrm{~Hz}, 1 \mathrm{H}), 3.81(\mathrm{dd}, J=10.6$ and $4.4 \mathrm{~Hz}, 1 \mathrm{H}), 3.72(\mathrm{dd}, J=10.5$ and $5.9 \mathrm{~Hz}$, $1 \mathrm{H}), 3.70(\mathrm{~m}, 1 \mathrm{H}), 3.60(\mathrm{dd}, J=9.3$ and $2.2 \mathrm{~Hz}, 1 \mathrm{H}), 3.48(\mathrm{~m}, 1 \mathrm{H}), 2.85(\mathrm{~d}, J=7.6 \mathrm{~Hz}, 1 \mathrm{H})$, $2.50(\mathrm{~d}, J=3.9 \mathrm{~Hz}, 1 \mathrm{H}) ; \mathbf{M S}(\mathrm{ES}) \mathrm{m} / \mathrm{z} 593(\mathrm{M}+\mathrm{Na})^{+}$.

Stereochemical assignment of alcohol 3: spin-coupling constants ${ }^{3} J_{\text {H-H }}$ and NOE observed

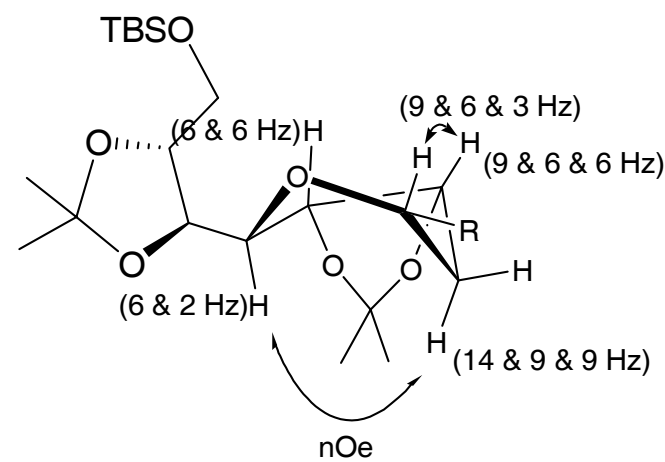<smiles>CC(C)=CCCC(C)(O)C(O)C=[18O]</smiles> 


\section{Mosher ester analysis of alcohol 3}

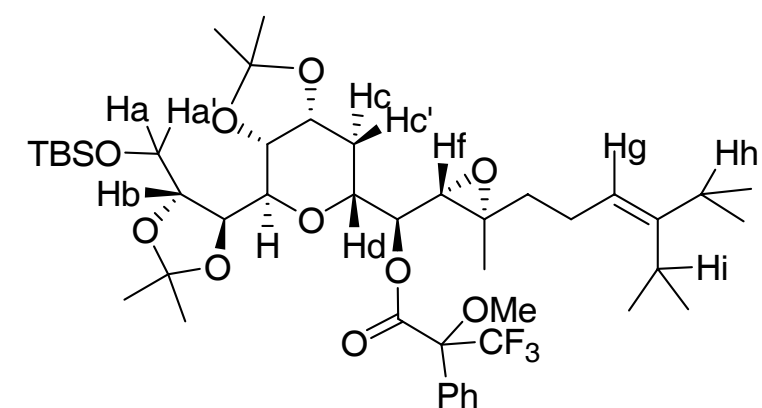

\begin{tabular}{cccc}
\hline & $\boldsymbol{\delta}(\boldsymbol{S}, \boldsymbol{R})$ & $\boldsymbol{\Delta}(\boldsymbol{S}, \boldsymbol{S})$ & $\boldsymbol{\delta}(\boldsymbol{S}, \boldsymbol{S})-\boldsymbol{\delta}(\boldsymbol{S}, \boldsymbol{R})^{\mathrm{a}}$ \\
\hline H-a & 3.73 & 3.71 & -0.02 \\
H-a' & 3.69 & 3.69 & 0 \\
H-b & 4.15 & 4.02 & -0.13 \\
H-c & 2.16 & 2.01 & -0.15 \\
H-c' & 1.87 & 2.01 & 0.14 \\
H-d & 4.14 & 4.1 & -0.04 \\
H-e & - & - & - \\
H-f & 2.75 & 2.99 & 0.24 \\
H-g & 4.96 & 5.08 & 0.12 \\
H-h & 1.65 & 1.67 & 0.02 \\
H-i & 1.54 & 1.58 & 0.04 \\
\hline
\end{tabular}

${ }^{a}$ Positive values for $\Delta \delta$ means that the signal is shifted downfield and negative values for $\Delta \delta$ means that the signal is shifted upfield from that of the other diastereomeric ester. 


\section{Reductive Lithiations of Phenylthio Acetals 5}

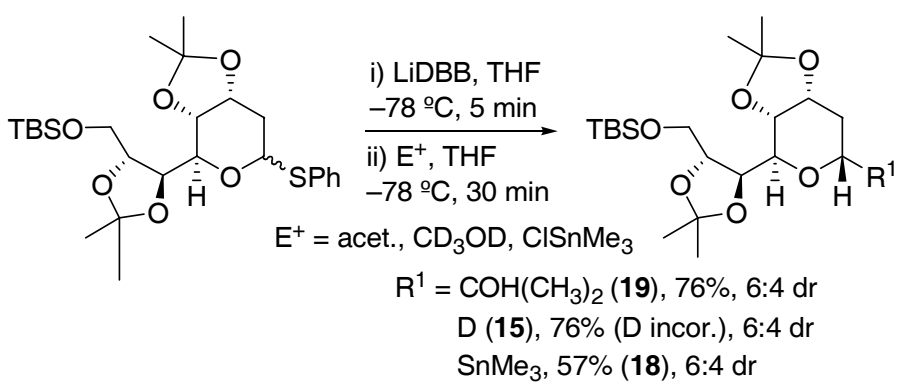

2-(3aS,4R,6S,7aR)- $\quad$ and $\quad 2-(3 \mathrm{a} S, 4 R, 6 R, 7 \mathrm{a} R)-4-[(4 R, 5 R)-5-([1-($ tert-Butyl)-1,1dimethylsilyl]oxymethyl)-2,2-dimethyl-1,3-dioxolan-4-yl]-2,2-

\section{dimethylperhydro[1,3]dioxolo[4,5-c]pyran-6-yl-2-propanol (19eq and 19ax)}
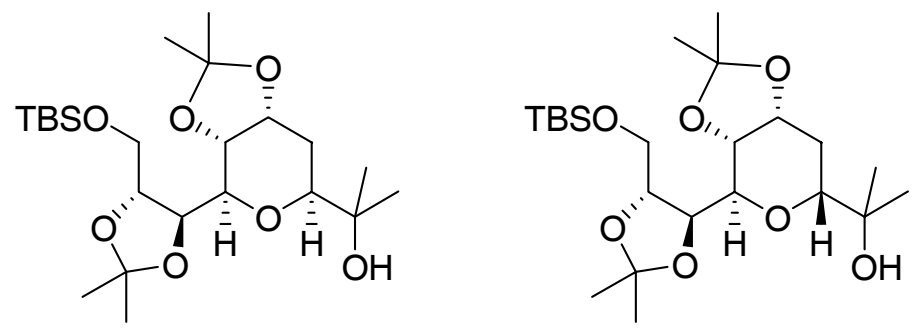

According to the reductive lithiation procedure described in the previous section, treatment of a 1:1 mixture of phenylthio acetals $5(74 \mathrm{mg}, 0.145 \mathrm{mmol})$ with LiDBB followed by reaction with acetone $(50 \mu \mathrm{L}, 0.68)$ and chromatography (eluent hexanes/ether 2:1) afforded a 60:40 mixture of isomers 19ax and 19eq (54 mg, 76\%). This mixture of isomers was separated by MPLC (eluent hexanes/ether 4:1).

19eq; colorless oil; $\mathrm{R}_{f}=0.30$ (eluent hexanes/ether 2:1); $[\boldsymbol{\alpha}]^{\mathbf{2 0}}{ }_{\mathbf{D}}+8.33\left(\mathrm{c} 0.3, \mathrm{CH}_{2} \mathrm{Cl}_{2}\right) ;{ }^{1} \mathbf{H}$ NMR $\left(500 \mathrm{MHz}, \mathrm{CDCl}_{3}\right) \delta 4.43(\mathrm{~m}, 1 \mathrm{H}), 4.29(\mathrm{ddd}, J=7.8,6.1$ and $4.4 \mathrm{~Hz}, 1 \mathrm{H}), 4.22(\mathrm{dd}, J=$ 9.3 and $5.1 \mathrm{~Hz}, 1 \mathrm{H}), 4.14(\mathrm{dd}, J=8.1$ and $2.5 \mathrm{~Hz}, 1 \mathrm{H}), 3.82(\mathrm{dd}, J=10.5$ and $4.4 \mathrm{~Hz}, 1 \mathrm{H})$, $3.73(\mathrm{dd}, J=10.3$ and $5.9 \mathrm{~Hz}, 1 \mathrm{H}), 3.61(\mathrm{dd}, J=9.5$ and $2.5 \mathrm{~Hz}, 1 \mathrm{H}), 3.46(\mathrm{dd}, J=12.0$ and 2.2 $\mathrm{Hz}, 1 \mathrm{H}), 2.27(\mathrm{~s}, 1 \mathrm{H}), 2.08(\mathrm{dt}, J=14.9$ and $2.1 \mathrm{~Hz}, 1 \mathrm{H}), 1.90(\mathrm{ddd}, J=15.6,12.0$ and $3.2 \mathrm{~Hz}$, $1 \mathrm{H}), 1.51(\mathrm{~s}, 3 \mathrm{H}), 1.45(\mathrm{~s}, 3 \mathrm{H}), 1.43(\mathrm{~s}, 3 \mathrm{H}), 1.37$ (s, $3 \mathrm{H}), 1.24(\mathrm{~s}, 3 \mathrm{H}), 1.16$ (s, $3 \mathrm{H}), 0.91$ (s, $9 \mathrm{H}), 0.09$ (s, $3 \mathrm{H}), 0.08$ (s, $3 \mathrm{H}) ;{ }^{13} \mathbf{C} \mathbf{N M R}\left(125 \mathrm{MHz}, \mathrm{CDCl}_{3}\right) \delta 109.3,108.9,80.4,78.9,77.3$, 76.7, 72.5, 71.4, 70.5, 64.4, 29.6, 28.2, 27.3, 27.1, 26.9, 26.5, 26.3, 25.9 (3 C), 24.1, -5.4, -5.4; IR $v_{\max } / \mathrm{cm}^{-1} 3470,2956,2931,1380,1250,1077,838,778 ;$ MS m/z (CI) $445\left(\mathrm{M}-\mathrm{CH}_{3}\right)^{+}$; HRMS (CI): $m / z$ calcd for $\mathrm{C}_{22} \mathrm{H}_{41} \mathrm{O}_{7} \mathrm{Si}: 445.2619$, found 445.2621 . 
19ax; colorless oil; $\mathrm{R}_{f}=0.35$ (eluent hexanes/ether 2:1); $[\boldsymbol{\alpha}]^{\mathbf{2 0}}{ }_{\mathbf{D}}+5.75\left(\mathrm{c} 0.4, \mathrm{CH}_{2} \mathrm{Cl}_{2}\right) ;{ }^{\mathbf{1}} \mathbf{H}$ NMR $\left(500 \mathrm{MHz}, \mathrm{CDCl}_{3}\right) \delta 4.36(\mathrm{dt}, J=10.6$ and $6.5 \mathrm{~Hz}, 1 \mathrm{H}), 4.26(\mathrm{t}, J=6.1 \mathrm{~Hz}, 1 \mathrm{H}), 4.22$ $(\mathrm{dd}, J=8.1$ and $2.4 \mathrm{~Hz}, 1 \mathrm{H}), 4.12(\mathrm{~m}, 1 \mathrm{H}), 4.06(\mathrm{dd}, J=6.1$ and $2.1 \mathrm{~Hz}, 1 \mathrm{H}), 3.82(\mathrm{dd}, J=$ 10.8 and $4.1 \mathrm{~Hz}, 1 \mathrm{H}), 3.76(\mathrm{dd}, J=10.9$ and $5.5 \mathrm{~Hz}, 1 \mathrm{H}), 3.52(\mathrm{dd}, J=12.5$ and $3.3 \mathrm{~Hz}, 1 \mathrm{H}$ ), $2.31(\mathrm{~s}, 1 \mathrm{H}), 1.99$ (ddd, $J=13.1,6.1$ and $3.3 \mathrm{~Hz}, 1 \mathrm{H}), 1.69(\mathrm{td}, J=12.7$ and $10.5 \mathrm{~Hz}, 1 \mathrm{H})$, 1.49 (s, $3 \mathrm{H}), 1.44$ (s, $3 \mathrm{H}), 1.42$ (s, $3 \mathrm{H}), 1.35$ (s, $3 \mathrm{H}), 1.21$ (s, $3 \mathrm{H}), 1.16$ (s, $3 \mathrm{H}), 0.91$ (s, $9 \mathrm{H})$, 0.08 (s, $6 \mathrm{H}) ;{ }^{13} \mathbf{C}$ NMR $\left(125 \mathrm{MHz}, \mathrm{CDCl}_{3}\right) \delta$ 109.6, 108.6, 80.2, 77.3, 77.0, 73.6, 72.5, 72.4, 71.1, 63.9, 28.1, 27.6, 27.2, 27.0, 26.1 (3C), 25.9, 25.4, 24.1, 18.4, -5.3, -5.3; IR $v_{\max } / \mathrm{cm}^{-1}$; MS $\mathrm{m} / \mathrm{z}(\mathrm{CI}) 445\left(\mathrm{M}-\mathrm{CH}_{3}\right)^{+}$; HRMS (CI): $\mathrm{m} / z$ calcd for $\mathrm{C}_{22} \mathrm{H}_{41} \mathrm{O}_{7} \mathrm{Si}$ : 445.2619, found 445.2621.

Stereochemical assignment of alcohol 19eq: spin-coupling constants ${ }^{3} J_{\mathrm{H}-\mathrm{H}}$ and NOE observed

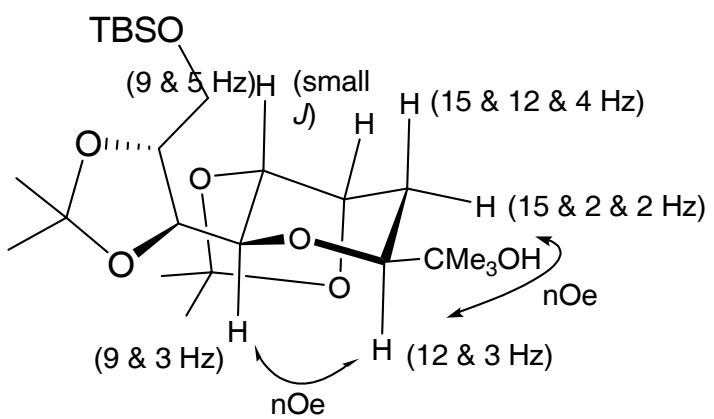

Stereochemical assignment of alcohol 19ax: spin-coupling constants ${ }^{3} J_{\mathrm{H}-\mathrm{H}}$ and NOE observed

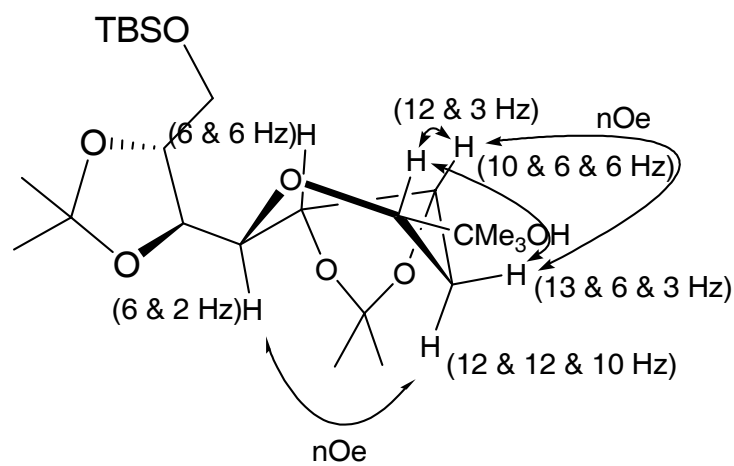

Modified procedure for the reductive lithiation of phenylthio acetals by inverse addition of LiDBB and subsequent addition of acetone

A solution of $n$-BuLi (2.4 M in hexanes, 2-3 drops) was added dropwise to a solution of phenylthio acetal 5ax $(26 \mathrm{mg}, 0.051 \mathrm{mmol})$ and a couple of crystals of 1,10-phenantroline in 
anhydrous THF $(2 \mathrm{~mL})$ at $-78^{\circ} \mathrm{C}$ until an orange-red coloration just appeared. This solution was added dropwise via cannula at $-78{ }^{\circ} \mathrm{C}$ to a freshly prepared solution of LiDBB $(0.3 \mathrm{M}$ in THF, $1.7 \mathrm{~mL}, 0.51 \mathrm{mmol}$ ) in THF. The resultant green solution was stirred at $-78{ }^{\circ} \mathrm{C}$ for $5 \mathrm{~min}$. Freshly distilled acetone $(75 \mu \mathrm{L}, 1.02 \mathrm{mmol})$ was added to the reaction mixture. After addition of acetone, the resultant orange solution was stirred at $-78{ }^{\circ} \mathrm{C}$ for $30 \mathrm{~min}$. The reaction was quenched by sequential addition of methanol $(1 \mathrm{~mL})$ and sat. aq. $\mathrm{NH}_{4} \mathrm{Cl}(1 \mathrm{~mL})$ at $-78{ }^{\circ} \mathrm{C}$. The reaction mixture was allowed to warm to $\mathrm{rt}$ and diluted with ether $(10 \mathrm{~mL})$. The organic phase was separated and the aqueous was extracted with ether $(10 \mathrm{~mL})$. The combined organic extracts were dried over $\mathrm{MgSO}_{4}$, filtered and evaporated under reduced pressure to give a pale yellow residue. This material was purified by column chromatography (hexanes/ether 4:1) to afford a 60:40 mixture of alcohols 19ax and 19eq.

This reaction was also performed with phenylthio acetal 5eq $(17 \mathrm{mg}, 0.033 \mathrm{mmol})$ affording a 59:41 mixture of alcohols 19ax and 19eq.

\section{Procedure for the reductive lithiation of phenylthioacetals 5 under equilibration conditions}

A solution of $n$-BuLi (2.4 M in hexanes, 2-3 drops) was added dropwise to a solution of a 1:1 mixture of phenylthio acetals $5(28 \mathrm{mg}, 0.055 \mathrm{mmol})$ and a couple of crystals of 1,10phenantroline in anhydrous THF $(2 \mathrm{~mL})$ at $-78^{\circ} \mathrm{C}$ until an orange-red coloration just appeared. A freshly prepared $0.3 \mathrm{M}$ solution of LiDBB in THF was added dropwise via a gas tight syringe until a permanent dark green color persisted. The resultant green solution was then left to warm up to $-50{ }^{\circ} \mathrm{C}$ (monitored by an internal thermometer) and stirred at that temperature for $30 \mathrm{~min}$. Freshly distilled acetone (50 $\mu \mathrm{L}, 0.680 \mathrm{mmol})$ was added to the reaction mixture. The resultant orange solution was stirred at $-78{ }^{\circ} \mathrm{C}$ for $30 \mathrm{~min}$. The reaction was quenched by sequential addition of methanol $(1 \mathrm{~mL})$ and sat. aq. $\mathrm{NH}_{4} \mathrm{Cl}(1 \mathrm{~mL})$ at $-78{ }^{\circ} \mathrm{C}$. The reaction mixture was allowed to warm to $\mathrm{rt}$ and diluted with ether $(10 \mathrm{~mL})$. The organic phase was separated and the aqueous was extracted with ether $(10 \mathrm{~mL})$. The combined organic extracts were dried over $\mathrm{MgSO}_{4}$, filtered and evaporated under reduced pressure to give a pale yellow residue. This material was purified by column chromatography (hexanes/ether 4:1) to afford a 60:40 mixture of alcohols 19ax and 19eq. 
$(4 R, 5 S)-5-[(3 \mathrm{a} R, 4 R, 6 R, 7 \mathrm{a} R)-\quad$ and $\quad(4 R, 5 S)-5-[(3 \mathrm{a} R, 4 R, 6 S, 7 \mathrm{a} R)-2,2-D i m e t h y l-6-(1,1,1-$ trimethylstannyl)perhydro[1,3]dioxolo[4,5-c]pyran-4-yl]-2,2-dimethyl-1,3-dioxolan-4ylmethoxy)(tert-butyl)dimethylsilane (18eq and 18ax)
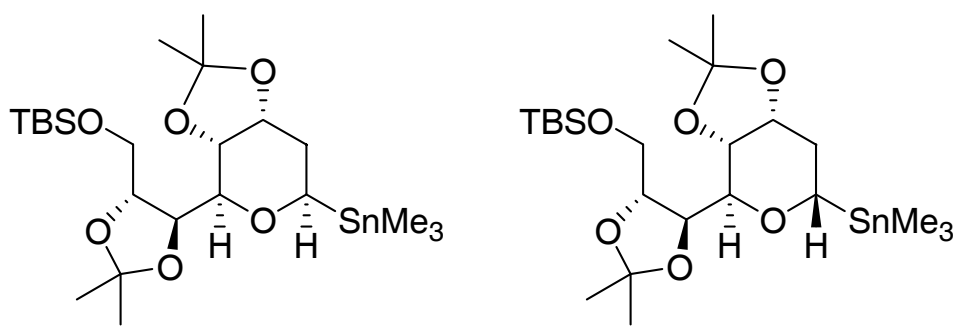

According to the reductive lithiation procedure described in the previous section, treatment of a 1:1 mixture of phenylthio acetals $5(93 \mathrm{mg}, 0.182 \mathrm{mmol})$ with LiDBB followed by reaction with trimethyltin chloride ( $1 \mathrm{M}$ in hexanes, $369 \mu \mathrm{L}, 0.369 \mathrm{mmol})$ and chromatography (eluent hexanes/ether 8:1) afforded 18ax (34 mg, 34\%) and 18eq (23 mg, $23 \%)$.

18eq; colorless oil; $\mathrm{R}_{f}=0.30$ (eluent hexanes/ether 8:1); $[\boldsymbol{\alpha}]^{\mathbf{2 0}}{ }_{\mathbf{D}}-28.5\left(\mathrm{c} 0.2, \mathrm{CH}_{2} \mathrm{Cl}_{2}\right) ;{ }^{1} \mathbf{H}$ NMR $\left(500 \mathrm{MHz}, \mathrm{CDCl}_{3}\right) \delta 4.30(\mathrm{~m}, 1 \mathrm{H}), 4.25(\mathrm{ddd}, J=7.6,5.9$ and $4.4 \mathrm{~Hz}, 1 \mathrm{H}), 4.17(\mathrm{dd}, J=$ 9.3 and $4.9 \mathrm{~Hz}, 1 \mathrm{H}), 4.06(\mathrm{dd}, J=7.6$ and $2.5 \mathrm{~Hz}, 1 \mathrm{H}), 3.81(\mathrm{dd}, J=13.2$ and $3.0 \mathrm{~Hz}, 1 \mathrm{H})$, $3.79(\mathrm{dd}, J=10.5$ and $4.4 \mathrm{~Hz}, 1 \mathrm{H}), 3.70(\mathrm{dd}, J=10.5$ and $5.9 \mathrm{~Hz}, 1 \mathrm{H}), 3.40(\mathrm{dd}, J=9.5$ and 2.7 $\mathrm{Hz}, 1 \mathrm{H}), 2.19(\mathrm{td}, J=15.2$ and $3.7 \mathrm{~Hz}, 1 \mathrm{H}), 2.12(\mathrm{dt}, J=15.2$ and $2.4 \mathrm{~Hz}, 1 \mathrm{H}), 1.52(\mathrm{~s}, 3 \mathrm{H})$, 1.43 (s, $3 \mathrm{H}), 1.41$ (s, $3 \mathrm{H}), 1.36$ (s, $3 \mathrm{H}), 0.91$ (s, $9 \mathrm{H}), 0.11$ (s, $9 \mathrm{H}), 0.09$ (s, $3 \mathrm{H}), 0.08$ (s, $3 \mathrm{H})$; ${ }^{13} \mathrm{C}$ NMR $\left(125 \mathrm{MHz}, \mathrm{CDCl}_{3}\right) \delta$ 109.3, 108.7, 80.5, 80.0, 77.1, 71.6, 71.6, 67.5, 64.6, 32.4, 28.3, 27.4, 26.9, 26.6, 25.9 (3 C), 18.4, -5.3, -5.4, -11.1 (3 C); IR $v_{\max } / \mathrm{cm}^{-1} 2984,2931,1461,1367$, 1244, 1217, 1067, 836, 779; MS (ES) m/z $589(\mathrm{M}+\mathrm{Na})^{+}, \mathbf{M S ~ m / z ~ ( C I ) ~} 551\left(\mathrm{M}-\mathrm{CH}_{3}\right)^{+}$; HRMS (CI): $m / z$, calcd for $\mathrm{C}_{22} \mathrm{H}_{45} \mathrm{O}_{6} \mathrm{SiSn}: 551.1851$, found 551.1837 .

18ax; colorless oil; $\mathrm{R}_{f}=0.25$ (eluent hexanes/ether 8:1); $[\boldsymbol{\alpha}]^{\mathbf{2 0}}{ }_{\mathbf{D}}+2.0\left(\mathrm{c} 0.35, \mathrm{CH}_{2} \mathrm{Cl}_{2}\right) ;{ }^{\mathbf{1}} \mathbf{H}$ NMR $\left(500 \mathrm{MHz}, \mathrm{C}_{6} \mathrm{D}_{6}\right) \delta 4.44(\mathrm{dd}, J=7.8$ and $4.4 \mathrm{~Hz}, 1 \mathrm{H}), 4.39(\mathrm{t}, J=5.4 \mathrm{~Hz}, 1 \mathrm{H}), 4.27$ (dt, $J$ $=4.2$ and $3.9 \mathrm{~Hz}, 1 \mathrm{H}), 4.17(\mathrm{dt}, J=7.3$ and $5.6 \mathrm{~Hz}, 1 \mathrm{H}), 4.05(\mathrm{dd}, J=9.5$ and $4.4 \mathrm{~Hz}, 1 \mathrm{H})$, $4.02(\mathrm{t}, J=5.1 \mathrm{~Hz}, 1 \mathrm{H}), 3.84(\mathrm{dd}, J=11.0$ and $3.9 \mathrm{~Hz}, 1 \mathrm{H}), 3.77(\mathrm{dd}, J=11.0$ and $3.9 \mathrm{~Hz}, 1$ H), 2.11 (ddd, $J=14.2,9.5$ and $7.6 \mathrm{~Hz}, 1 \mathrm{H}), 1.93$ (ddd, $J=14.0,5.9$ and $4.6 \mathrm{~Hz}, 1 \mathrm{H}), 1.52$ (s, 3 H), 1.45 (s, 3 H), 1.43 (s, 3 H), 1.29 (s, 3 H), 0.98 (s, 9 H), 0.19 (s, 9 H), 0.13 (s, 3 H), 0.08 (s, 3 $\mathrm{H}) ;{ }^{13} \mathrm{C}$ NMR $\left(125 \mathrm{MHz}, \mathrm{C}_{6} \mathrm{D}_{6}\right) \delta 109.3,108.2,79.3,78.1,76.0,72.3,71.8,66.7,64.0,33.1$, 28.5, 27.6, 27.2, 26.2, 26.1 (3 C), 18.2, -5.1, -5.2, -10.2 (3 C); IR $v_{\max } / \mathrm{cm}^{-1} 2985,1462,1368$, 
1248, 1216, 1073, 836, 776; MS (ES) m/z $589(\mathrm{M}+\mathrm{Na})^{+}, \mathbf{M S ~ m / z ~ ( C I ) ~} 551\left(\mathrm{M}-\mathrm{CH}_{3}\right)^{+}$; HRMS (CI): $m / z$ calcd for $\mathrm{C}_{22} \mathrm{H}_{45} \mathrm{O}_{6} \mathrm{SiSn}: 551.1851$, found 551.1846 .

Stereochemical assignment of stannane 18eq: spin-coupling constants ${ }^{3} J_{\mathrm{H}-\mathrm{H}}$ and NOE observed

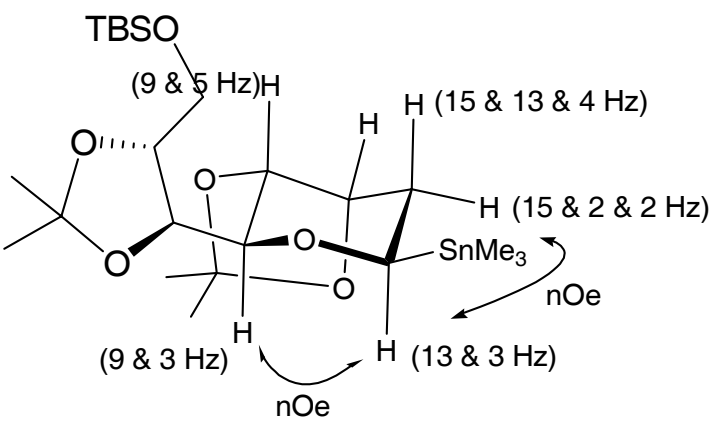

Stereochemical assignment of stannane 18ax: spin-coupling constants ${ }^{3} J_{\mathrm{H}-\mathrm{H}}$ and NOE observed

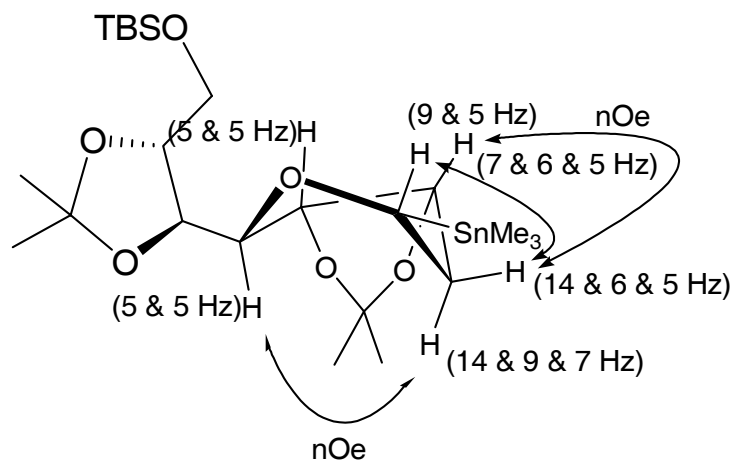

$(4 R, 5 R)-5$-[(3aS,4R,7aR)-2,2-Dimethylperhydro[1,3]dioxolo[4,5-c]pyran-4-yl]-2,2-dimethyl1,3-dioxolan-4-ylmethoxy)(tert-butyl)dimethylsilane (34)<smiles>CC(C)(C)OC[C@H]1OC(C)(C)O[C@H]1[C@H]1OCC[C@@H]2OC(C)(C)O[C@H]21</smiles>

According to the reductive lithiation procedure described in the previous section, treatment of a 1:1 mixture of phenylthio acetals $5(25 \mathrm{mg}, 0.049 \mathrm{mmol})$ with LiDBB followed by reaction with methanol $(1 \mathrm{~mL})$ and chromatography (eluent hexanes/ether $4: 1)$ afforded tetrahydropyran 34 (17 mg, 90\%). This reaction was also carried out using $\mathrm{CD}_{3} \mathrm{OD}$ as 
electrophile providing a 6:4 mixture of tetrahydropyrans $\mathbf{1 5}$ in favour of $\mathbf{1 5 a x}$ with a $76 \%$ deuterium incorporation measured by ${ }^{1} \mathrm{H}$ NMR.

34; colorless oil; $\mathrm{R}_{f}=0.35$ (eluent hexanes/ether 4:1); $[\boldsymbol{\alpha}]^{\mathbf{2 0}}{ }_{\mathrm{D}}-12.7\left(\mathrm{c} 0.45, \mathrm{CH}_{2} \mathrm{Cl}_{2}\right) ;{ }^{1} \mathbf{H}$ NMR $\left(500 \mathrm{MHz}, \mathrm{CDCl}_{3}\right) \delta 4.40(\mathrm{~m}, 1 \mathrm{H}), 4.31-4.26(\mathrm{~m}, 2 \mathrm{H}), 4.16(\mathrm{dd}, J=8.3$ and $2.4 \mathrm{~Hz}, 1$ H), $3.84(\mathrm{ddd}, J=11.5,5.6$ and $1.7 \mathrm{~Hz}, 1 \mathrm{H}), 3.79(\mathrm{dd}, J=10.5$ and $4.2 \mathrm{~Hz}, 1 \mathrm{H}), 3.74(\mathrm{dd}, J=$ 10.5 and $5.4 \mathrm{~Hz}, 1 \mathrm{H}), 3.63(\mathrm{td}, J=11.7$ and $3.2 \mathrm{~Hz}, 1 \mathrm{H}), 3.46(\mathrm{dd}, J=8.8$ and $2.2 \mathrm{~Hz}, 1 \mathrm{H})$, $2.08(\mathrm{tdd}, J=14.9,5.6$ and $3.7 \mathrm{~Hz}, 1 \mathrm{H}), 2.02(\mathrm{dtd}, J=14.7,4.2$ and $2.4 \mathrm{~Hz}, 1 \mathrm{H}), 1.50$ (s, $3 \mathrm{H})$, 1.46 (s, $3 \mathrm{H}), 1.42$ (s, $3 \mathrm{H}), 1.37$ (s, $3 \mathrm{H}), 0.91$ (s, $9 \mathrm{H}), 0.09$ (s, $3 \mathrm{H}), 0.08$ (s, $3 \mathrm{H})$; ${ }^{13} \mathbf{C}$ NMR $\left(125 \mathrm{MHz}, \mathrm{CDCl}_{3}\right) \delta$ 109.2, 108.8, 79.8, 76.9 (2 C), 71.6, 70.4, 64.0, 63.1, 28.3, 27.4, 27.2, 26.9, 26.4, 25.9 (3 C), 18.4, -5.3, -5.4,; IR $v_{\max } / \mathrm{cm}^{-1}$ 2986, 2995, 2932, 2860, 1464, 1380, 1370, 1250, 1218, 1164, 1142, 1087, 1046, 838, 778; MS (ES) m/z $425(\mathrm{M}+\mathrm{Na})^{+}, \mathrm{MS} \mathrm{m} / \mathrm{z}(\mathrm{CI}) 403$ $(\mathrm{M}+\mathrm{H})^{+} ;$HRMS (CI): $\mathrm{m} / z$ calcd for $\mathrm{C}_{20} \mathrm{H}_{39} \mathrm{O}_{6} \mathrm{Si}: 403.2516$, found 403.2517 .

\section{Tin-lithium exchange and alkylation of stannanes 18ax and 18eq}

A solution of $n$-BuLi (2.5 M in hexanes, $42 \mu \mathrm{L}, 0.106 \mathrm{mmol})$ was added dropwise to a solution of 2-trimethylstannyltetrahydropyran $\mathbf{1 8 e q}(12 \mathrm{mg}, 0.021 \mathrm{mmol})$ in anhydrous THF (1 $\mathrm{mL})$ at $-78^{\circ} \mathrm{C}$. The resultant mixture was stirred at $-78^{\circ} \mathrm{C}$ for $5 \mathrm{~min}$ and acetone $(80 \mu \mathrm{L}, 0.424)$ was added. After stirring at $-78{ }^{\circ} \mathrm{C}$ for $30 \mathrm{~min}$, the reaction was quenched by sequential addition of methanol $(1 \mathrm{~mL})$ and sat. aq. $\mathrm{NH}_{4} \mathrm{Cl}(1 \mathrm{~mL})$ at $-78^{\circ} \mathrm{C}$. The reaction mixture was allowed to warm to $\mathrm{rt}$ and diluted with ether $(10 \mathrm{~mL})$. The organic phase was separated and the aqueous layer extracted with ether $(10 \mathrm{~mL})$. The combined organic extracts were dried over $\mathrm{MgSO}_{4}$, filtered and evaporated under reduced pressure to give a colorless oil. ${ }^{1} \mathrm{H}$ NMR analysis of the crude product showed almost pure alcohol 19eq contaminated with small amount of the corresponding protonated product $\mathbf{3 4}$.

The above procedure was also applied to a microscale transmetallation of 2trimethylstannyltetrahydropyran 18ax providing almost pure alcohol 19ax contaminated with a small amount of the corresponding protonated product $\mathbf{3 4}$. 


\section{Synthesis of Phenylthio Acetals 20, 21 and 22}

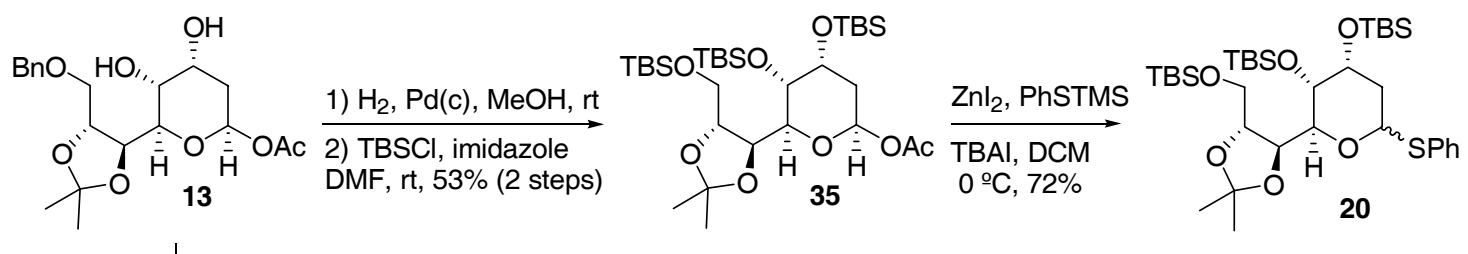

1) $\mathrm{Ac}_{2} \mathrm{O}$, pyridine, rt, $92 \%(36)$ 2) $\mathrm{H}_{2}, \mathrm{Pd}(\mathrm{c}), \mathrm{MeOH}, \mathrm{rt}, 94 \%$

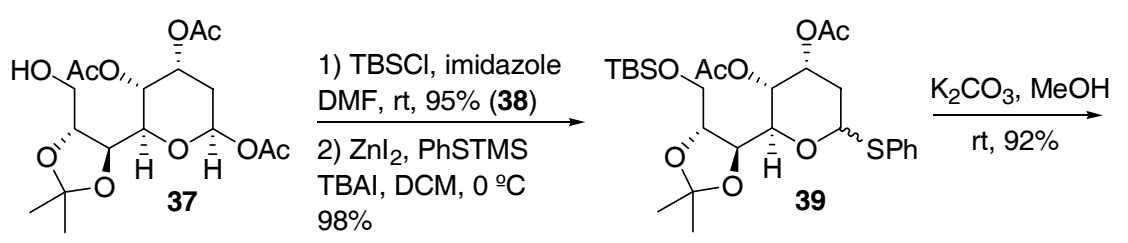<smiles>CC(C)(C)OC[C@H]1OC(C)(C)O[C@H]1[C@H]1O[C@H](c2ccccc2)C[C@H](O)[C@H]1O</smiles>

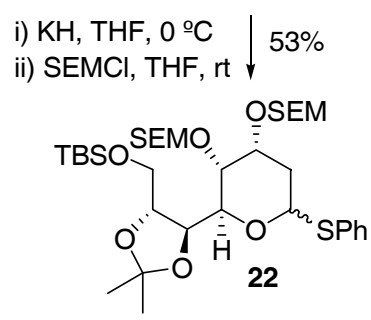

$(2 R, 4 R, 5 S, 6 S)-4,5-D i[1-(t e r t-b u t y l)-1,1-d i m e t h y l s i l y l] o x y-6-[(4 R, 5 R)-5-([1-(t e r t-b u t y l)-1,1-$ dimethylsilyl]oxymethyl)-2,2-dimethyl-1,3-dioxolan-4-yl]tetrahydro-2H-2-pyranyl acetate (35)

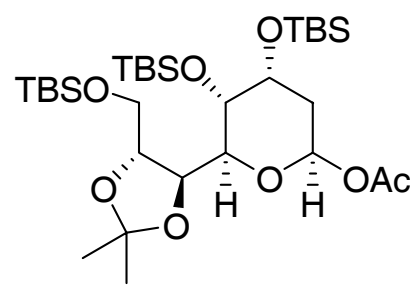

A solution of diol 13 (288 $\mathrm{mg}, 0,72 \mathrm{mmol})$ in methanol $(9 \mathrm{~mL})$ was added under argon to a flask containing palladium $10 \%$ on activated carbon $(35 \mathrm{mg})$. The heterogeneous mixture was purged with $\mathrm{H}_{2}$ and stirred under $\mathrm{H}_{2}$ at $\mathrm{rt}$ for $54 \mathrm{~h}$. The reaction mixture was then purged with argon, diluted with DCM $(80 \mathrm{~mL})$ and filtered through a celite ${ }^{\circledR}$ plug. The filtrated was evaporated under reduced pressure to provide the crude oil. This crude oil was partially purified by flash chromatography (ethyl acetate) providing the triol (191 $\mathrm{mg}, 86 \%$ ) as a colorless oil. This compound was found to be unstable in $\mathrm{SiO}_{2}$, it was therefore used in the following synthetic step without further purification. $\mathrm{R}_{f}=0.15$ (eluent DCM/MeOH 98:2); ${ }^{1} \mathbf{H}$ NMR (500 $\left.\mathrm{MHz}, \mathrm{CDCl}_{3}\right) \delta 5.96(\mathrm{dd}, J=10.0$ and $2.2 \mathrm{~Hz}, 1 \mathrm{H}), 4.18(\mathrm{q}, J=3.2 \mathrm{~Hz}, 1 \mathrm{H}), 4.11(\mathrm{dt}, J=7.8$ 
and $4.2 \mathrm{~Hz}, 1 \mathrm{H}), 3.89(\mathrm{t}, J=8.3 \mathrm{~Hz}, 1 \mathrm{H}), 3.80(\mathrm{~m}, 1 \mathrm{H}), 3.78(\mathrm{~m}, 1 \mathrm{H}), 3.67(\mathrm{dd}, J=9.3$ and 3.2 $\mathrm{Hz}, 1 \mathrm{H}), 3.63(\mathrm{dd}, J=12.0$ and $3.9 \mathrm{~Hz}, 1 \mathrm{H}), 2.90$ (bs, $1 \mathrm{H}), 2.41$ (bs, $1 \mathrm{H}), 2.12$ (dd, $J=13.5$, 3.4 and $3.2 \mathrm{~Hz}, 1 \mathrm{H}), 2.05$ (s, $3 \mathrm{H}), 1.76(\mathrm{ddd}, J=13.5,10.0$ and $2.7 \mathrm{~Hz}, 1 \mathrm{H}), 1.43(\mathrm{~s}, 3 \mathrm{H}), 1.41$ (s, $3 \mathrm{H}$ ); HRMS (ES): $\mathrm{m} / z$ calcd for $\mathrm{C}_{13} \mathrm{H}_{22} \mathrm{O}_{8}: 306.1315$, found 329.1211 .

A solution of the above triol $(191 \mathrm{mg}, 0.62 \mathrm{mmol}), \mathrm{TBSCl}(1.67 \mathrm{~g}, 11.11 \mathrm{mmol})$ and imidazole (1.51 g, $22.22 \mathrm{mmol})$ in anhydrous DMF (2 mL) was stirred at $\mathrm{rt}$ for $90 \mathrm{~h}$. Ether (100 $\mathrm{mL})$ and water $(100 \mathrm{~mL})$ were added. The organic phase was separated and the aqueous was extracted with ether $(100 \mathrm{~mL})$. The combined organic phases were dried over $\mathrm{MgSO}_{4}$, filtered and evaporated under reduced pressure to give a colorless oil. This crude oil was purified by column chromatography (hexanes/ether 9:1) to provide acetoxy ether 35 (257 $\mathrm{mg}, 62 \%)$ as a white solid. $\mathrm{R}_{f}=0.20$ (eluent hexanes/ether 9:1); m.p. 43-45 ${ }^{\circ} \mathrm{C} ;[\boldsymbol{\alpha}]^{\mathbf{2 0}}{ }_{\mathrm{D}}+19.45\left(\mathrm{c} 0.55, \mathrm{CH}_{2} \mathrm{Cl}_{2}\right)$; ${ }^{1} \mathbf{H}$ NMR $\left(500 \mathrm{MHz}, \mathrm{CDCl}_{3}\right) \delta 6.11(\mathrm{dd}, J=4.9$ and $3.4 \mathrm{~Hz}, 1 \mathrm{H}), 4.13(\mathrm{ddd}, J=8.8,3.7$ and 2.0 $\mathrm{Hz}, 1 \mathrm{H}), 4.01(\mathrm{ddd}, J=7.0,5.1$ and $2.5 \mathrm{~Hz}, 1 \mathrm{H}), 3.85-3.95(\mathrm{~m}, 3 \mathrm{H}), 3.84(\mathrm{dd}, J=11.2$ and 2.7 $\mathrm{Hz}, 1 \mathrm{H}), 3.63(\mathrm{dd}, J=11.2$ and $5.1 \mathrm{~Hz}, 1 \mathrm{H}), 2.18$ (ddd, $J=12.5,8.6$ and $2.9 \mathrm{~Hz}, 1 \mathrm{H}$ ), 2.05 (s, $3 \mathrm{H}), 1.70$ (ddd, $J=13.0,9.0$ and $4.2 \mathrm{~Hz}, 1 \mathrm{H}), 1.39$ (s, $3 \mathrm{H}), 1.37$ (s, $3 \mathrm{H}), 0.90$ (s, $27 \mathrm{H}), 0.09$ $(\mathrm{s}, 9 \mathrm{H}), 0.08(\mathrm{~s}, 3 \mathrm{H}), 0.07$ (s, $3 \mathrm{H}), 0.06$ (s, $3 \mathrm{H}) ;{ }^{13} \mathbf{C}$ NMR (125 MHz, $\left.\mathrm{CDCl}_{3}\right) \delta$ 169.2, 109.4, 92.9, 81.6, 81.0, 76.3, 71.1, 66.1, 63.9, 34.4, 27.2, 27.1, 26.0 (3 C), 25.9 (6 C), 21.2, 19.4, 18.3, 18.1, -4.1, -4.5, -4.6, -4.8, -5.1, -5.2.; IR $v_{\max } / \mathrm{cm}^{-1} 2894,1756,1472,1371,1256,842$; MS m/z (ES) $671(\mathrm{M}+\mathrm{Na})^{+} ;$HRMS (ES): $\mathrm{m} / z$ calcd for $\mathrm{C}_{31} \mathrm{H}_{64} \mathrm{O}_{8} \mathrm{Si}_{3} \mathrm{Na}: 671.3807$, found 671.3813 .

tert-Butyl $((4 R, 5 S)-5-[(2 S, 3 S, 4 R, 6 R)-$ and tert-Butyl $((4 R, 5 S)-5-[(2 S, 3 S, 4 R, 6 S)-3,4-d i[1-(t e r t-$ butyl)-1,1-dimethylsilyl]oxy-6-(phenylsulfanyl)tetrahydro-2H-2-pyranyl]-2,2-dimethyl-1,3dioxolan-4-ylmethoxy)dimethylsilane (20eq and 20ax)
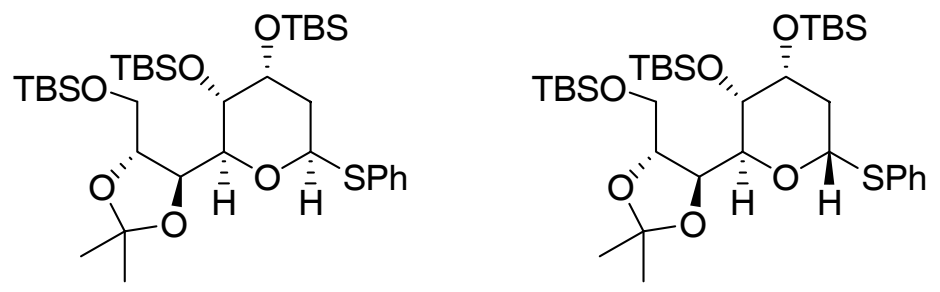

Phenylthiotrimethylsilane (306 $\mu \mathrm{L}, 1.62 \mathrm{mmol})$ was added to a solution of acetoxy ether 35 (210 mg, $0.32 \mathrm{mmol})$ at rt. $n$-Tetrabutylammonium iodide $(143 \mathrm{mg}, 0.38 \mathrm{mmol})$ was added and the reaction mixture was cooled to $0{ }^{\circ} \mathrm{C}$. $\mathrm{ZnI}_{2}(310 \mathrm{mg}, 0.97 \mathrm{mmol})$ was then added and the resultant mixture was stirred at $0{ }^{\circ} \mathrm{C}$ until no starting material was detected by ES analysis of aliquots (normally $30 \mathrm{~min}$ ). The reaction mixture was diluted with DCM (50 mL) and treated 
with $0.5 \mathrm{~N} \mathrm{NaOH}(15 \mathrm{~mL})$. The organic phase was separated and washed with brine $(20 \mathrm{~mL})$. The organic phase was then dried over $\mathrm{MgSO}_{4}$, filtered and evaporated under reduced pressure to give the crude product, which was purified by column chromatography (hexanes/ether 10:1) to afford phenylthio acetals $20(160 \mathrm{mg}, 72 \%)$ as an inseparable mixture of isomers. These compounds were characterized as 1:1 mixture of isomers as follows: ${ }^{1} \mathbf{H}$ NMR $\left(500 \mathrm{MHz}, \mathrm{C}_{6} \mathrm{D}_{6}\right.$, $\left.50{ }^{\circ} \mathrm{C}\right) \delta 7.59(\mathrm{bd}, J=8.3 \mathrm{~Hz}, 1 \mathrm{H}), 7.54(\mathrm{bd}, J=8.3 \mathrm{~Hz}, 1 \mathrm{H}), 7.05-7.10(\mathrm{~m}, 4 \mathrm{H}), 7.00(\mathrm{tt}, J=$ 6.8 and $1.2 \mathrm{~Hz}, 1 \mathrm{H}), 6.96(\mathrm{tt}, J=7.1$ and $1.2 \mathrm{~Hz}, 1 \mathrm{H}), 5.50(\mathrm{dd}, J=10.6$ and $3.2 \mathrm{~Hz}, 1 \mathrm{H}), 5.32$ $(\mathrm{dd}, J=7.8$ and $5.4 \mathrm{~Hz}, 1 \mathrm{H}), 4.35(\mathrm{dd}, J=8.6$ and $3.7 \mathrm{~Hz}, 1 \mathrm{H}), 4.31(\mathrm{t}, J=7.3 \mathrm{~Hz}, 1 \mathrm{H}), 4.24$ (ddd, $J=7.3,3.9$ and $2.7 \mathrm{~Hz}, 1 \mathrm{H}), 4.17$ (t, $J=7.3 \mathrm{~Hz}, 1 \mathrm{H}), 4.11(\mathrm{~m}, 1 \mathrm{H}), 4.14-4.18(\mathrm{~m}, 1 \mathrm{H})$, $4.06(\mathrm{t}, J=7.1 \mathrm{~Hz}, 1 \mathrm{H}), 4.07-4.09$ (m, $1 \mathrm{H}), 3.99-4.03$ (m, $2 \mathrm{H}), 3.99$ (dd, $J=11.2$ and $2.7 \mathrm{~Hz}$, $1 \mathrm{H}), 3.82-3.86(\mathrm{~m}, 2 \mathrm{H}), 3.72-3.76(\mathrm{~m}, 1 \mathrm{H}), 3.68(\mathrm{dd}, J=11.2$ and $3.4 \mathrm{~Hz}, 1 \mathrm{H}), 2.37(\mathrm{ddd}, J=$ 12.9, 9.3 and $7.8 \mathrm{~Hz}, 1 \mathrm{H}), 2.12(\mathrm{ddd}, J=13.2,5.1$ and $3.2 \mathrm{~Hz}, 1 \mathrm{H}), 1.99(\mathrm{dt}, J=12.9$ and 4.4 Hz, $1 \mathrm{H}$ ), 1.89 (ddd, $J=13.9,11.2$ and $3.7 \mathrm{~Hz}, 1 \mathrm{H}), 1.44$ (s, $3 \mathrm{H}), 1.40$ (s, $3 \mathrm{H}), 1.38$ (s, $3 \mathrm{H}$ ), 1.37 (s, $3 \mathrm{H}), 1.07$ (s, $9 \mathrm{H}), 1.02$ (s, $18 \mathrm{H}), 1.01$ (s, $9 \mathrm{H}), 0.99$ (s, $9 \mathrm{H}), 0.93$ (s, $9 \mathrm{H}), 0.24$ (s, 3 H), 0.21 (s, 3 H), 0.20 (s, 3 H), 0.15 (s, 3 H), 0.13 (s, 3 H), 0.13 (s, 6 H), 0.12 (s, 3 H), 0.11 (s, 3 $\mathrm{H}), 0.10(\mathrm{~s}, 3 \mathrm{H}), 0.08(\mathrm{~s}, 3 \mathrm{H}), 0.04(\mathrm{~s}, 3 \mathrm{H}) ;{ }^{13} \mathbf{C} \mathbf{N M R}\left(500 \mathrm{MHz}, \mathrm{C}_{6} \mathrm{D}_{6}, 50{ }^{\circ} \mathrm{C}\right) \delta 135.8,135.2$, 132.6 (2 C), 131.6 (2 C), 129.1 (2 C), 128.9 (2 C), 127.4, 127.3, 109.5, 109.4, 81.8, 81.6, 81.5, $80.4,77.0,75.3,73.9,71.9,70.3,69.2,64.3,63.3,35.7,30.1,27.4,27.4,27.4,27.3,26.5$ (3 C), 26.4 (3 C), 26.4 (3 C), 26.3 (3 C), 26.2 (3 C), 26.1 (3 C), 18.7, 18.6, 18.6, 18.5, 18.4, 18.4, -3.5, $-3.8,-4.1,-4.2,-4.2,-4.3,-4.3,-4.5,-4.9,-4.9,-5.0,-5.1 ; \mathrm{MS} \mathrm{m} / \mathrm{z}(\mathrm{ES}) 721(\mathrm{M}+\mathrm{Na})^{+}$; HRMS (ES): $m / z$ calcd for $\mathrm{C}_{35} \mathrm{H}_{66} \mathrm{O}_{6} \mathrm{SSi}_{3} \mathrm{Na}$ : 721.3785, found 721.3803 .

$(2 R, 3 S, 4 R, 6 S)-3,6-D i($ acetyloxy )-2-(4R,5R)-5-[(benzyloxy)methyl]-2,2-dimethyl-1,3dioxolan-4-yltetrahydro-2H-4-pyranyl acetate (36)

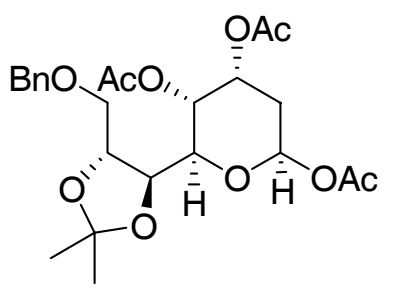

Acetic anhydride $(3.4 \mathrm{~mL})$ was added to a solution of diol $13(956 \mathrm{mg}, 2.41 \mathrm{mmol})$ in pyridine $(10 \mathrm{~mL})$ at $\mathrm{rt}$ under argon. After stirring at $\mathrm{rt}$ for $16 \mathrm{~h}$, the reaction solution was diluted with DCM, and the reaction was quenched at $0{ }^{\circ} \mathrm{C}$ by dropwise addition of sat. aq. $\mathrm{NaHCO}_{3}$ until basic $\mathrm{pH}$. The organic phase was separated and the aqueous was extracted with DCM $(2 \times 100$ $\mathrm{mL}$ ). The combined organic extracts were dried over $\mathrm{MgSO}_{4}$, filtered and evaporated under 
reduced pressure to give a yellow oil. This oil was purified by column chromatography (hexanes/ethyl acetate $4: 1)$ to give $36(1.11 \mathrm{~g}, 96 \%)$ as a colorless oil. $\mathrm{R}_{f}=0.25$ (eluent hexanes/ethyl acetate 4:1); $[\boldsymbol{\alpha}]^{\mathbf{2 0}}{ }_{\mathbf{D}}+16.48\left(\mathrm{c} 1.65, \mathrm{CH}_{2} \mathrm{Cl}_{2}\right) ;{ }^{1} \mathbf{H} \mathbf{N M R}\left(500 \mathrm{MHz}, \mathrm{CDCl}_{3}\right) \delta 7.23-$ $7.33(\mathrm{~m}, 5 \mathrm{H}), 6.02(\mathrm{dd}, J=8.8$ and $2.7 \mathrm{~Hz}, 1 \mathrm{H}), 5.50(\mathrm{dt}, J=5.9$ and $2.9 \mathrm{~Hz}, 1 \mathrm{H}), 5.10(\mathrm{dd}, J=$ 8.8 and $3.2 \mathrm{~Hz}, 1 \mathrm{H}), 4.58$ and 4.55 (AB system, $J=12.3 \mathrm{~Hz}, 2 \mathrm{H}), 4.25(\mathrm{~m}, 1 \mathrm{H}), 4.12(\mathrm{dd}, J=$ 8.8 and $4.4 \mathrm{~Hz}, 1 \mathrm{H}), 4.03$ (dd, $J=7.6$ and $4.4 \mathrm{~Hz}, 1 \mathrm{H}), 3.53-3.61$ (m, $2 \mathrm{H}), 2.09$ (s, 6H), 2.08 $(\mathrm{m}, 1 \mathrm{H}), 2.04(\mathrm{~s}, 3 \mathrm{H}), 1.96(\mathrm{~m}, 1 \mathrm{H}), 1.38(\mathrm{~s}, 3 \mathrm{H}), 1.36(\mathrm{~s}, 3 \mathrm{H}) ;{ }^{13} \mathbf{C} \mathbf{N M R}\left(125 \mathrm{MHz}, \mathrm{CDCl}_{3}\right)$ $\delta$ 169.8, 169.6, 169.0, 137.9, 128.3 (3 C), 127.5 (2 C), 109.9, 90.7, 78.0, 77.4, 73.4, 73.3, 70.8, 67.8, 66.6, 33.3, 27.0, 26.8, 20.9, 20.9, 20.7; IR $v_{\max } / \mathrm{cm}^{-1} 2938,1749,1370,1222,1062$; MS m/z (ES) $503(\mathrm{M}+\mathrm{Na})^{+} ;$HRMS (ES): $m / z$ calcd for $\mathrm{C}_{24} \mathrm{H}_{32} \mathrm{O}_{10} \mathrm{Na}$ : 503.1893, found 503.1891.

\section{$(2 R, 3 S, 4 R, 6 S)-3,6-D i(a c e t y l o x y)-2-[(4 R, 5 R)-5$-(hydroxymethyl)-2,2-dimethyl-1,3-dioxolan-}

\section{4-yl]tetrahydro-2H-4-pyranyl acetate (37)}

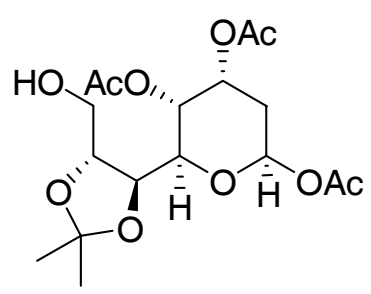

A solution of benzyl ether $36(1.05 \mathrm{~g}, 2.19 \mathrm{mmol})$ in methanol $(20 \mathrm{~mL})$ was added under argon to a flask containing palladium $10 \%$ on activated carbon $(250 \mathrm{mg})$. The heterogeneous mixture was purged with $\mathrm{H}_{2}$ and stirred under $\mathrm{H}_{2}$ at $\mathrm{rt}$ for $24 \mathrm{~h}$. The reaction mixture was then purged with argon, diluted with DCM $(80 \mathrm{~mL})$ and filtered through a celite ${ }^{\circledR}$ plug. The filtrated was evaporated under reduced pressure to provide the crude material, which was purified by column chromatography (hexanes/ethyl acetate 1:1) to give alcohol $37(802 \mathrm{mg}, 94 \%)$ as a colorless oil. $\mathrm{R}_{f}=0.25$ (eluent hexanes/ethyl acetate $1: 1$ ); $[\boldsymbol{\alpha}]^{\mathbf{2 0}}{ }_{\mathbf{D}}+20.06\left(\mathrm{c} 1.65, \mathrm{CH}_{2} \mathrm{Cl}_{2}\right) ;{ }^{1} \mathbf{H}$ NMR $\left(500 \mathrm{MHz}, \mathrm{CDCl}_{3}\right) \delta 6.05(\mathrm{dd}, J=8.1$ and $2.5 \mathrm{~Hz}, 1 \mathrm{H}), 5.47(\mathrm{dt}, J=6.1$ and $3.2 \mathrm{~Hz}, 1 \mathrm{H})$, $5.15(\mathrm{dd}, J=7.6$ and $3.2 \mathrm{~Hz}, 1 \mathrm{H}), 4.12(\mathrm{dd}, J=7.4$ and $4.0 \mathrm{~Hz}, 1 \mathrm{H}), 4.02-4.08(\mathrm{~m}, 2 \mathrm{H}), 3.78$ $(\mathrm{dt}, J=11.8$ and $4.2 \mathrm{~Hz}, 1 \mathrm{H}), 3.60(\mathrm{ddd}, J=11.8,7.6$ and $4.2 \mathrm{~Hz}, 1 \mathrm{H}) 2.10-2.15(\mathrm{~m}, 1 \mathrm{H}), 2.11$ (s, $3 \mathrm{H}), 2.10$ (s, $3 \mathrm{H}), 2.03$ (s, $3 \mathrm{H}), 1.97$ (ddd, $J=14.0,8.2$ and $3.2 \mathrm{~Hz}, 1 \mathrm{H}), 1.38(\mathrm{~s}, 3 \mathrm{H}), 1.37$ $(\mathrm{s}, 3 \mathrm{H}) ;{ }^{13} \mathrm{C}$ NMR $\left(125 \mathrm{MHz}, \mathrm{CDCl}_{3}\right) \delta 169.8,169.7,169.2,109.6,90.8,79.6,76.7,76.6,74.3$, 68.0, 66.3, 62.4, 32.9, 27.0, 26.8, 20.9, 20.7; IR $v_{\max } / \mathrm{cm}^{-1} 3505,2988,1748,1372,1226,1064$; MS m/z (ES) $413(\mathrm{M}+\mathrm{Na})^{+}$; HRMS (ES): $m / z$ calcd for $\mathrm{C}_{17} \mathrm{H}_{26} \mathrm{O}_{10} \mathrm{Na}$ : 413.1424, found 413.1439 . 
$(2 R, 3 S, 4 R, 6 S)-3,6-D i($ acetyloxy)-2-[(4R,5R)-5-([1-(tert-butyl)-1,1-dimethylsilyl]oxymethyl)2,2-dimethyl-1,3-dioxolan-4-yl]tetrahydro-2H-4-pyranyl acetate (38)<smiles>CC(=O)OC1C[C@H](OC(C)=O)[C@H]2OC(=O)C(C(C)C)[C@H]2OC(C)(C)O1</smiles>

A solution of alcohol 37 (776 mg, $1.99 \mathrm{mmol})$, TBSCl (450 mg, $2.99 \mathrm{mmol})$ and imidazole (676 mg, $9.93 \mathrm{mmol}$ ) in anhydrous DMF (15 mL) was stirred at rt for $14 \mathrm{~h}$. Ether (200 $\mathrm{mL})$ and water $(200 \mathrm{~mL})$ were added. The organic phase was separated and the aqueous was extracted with ether $(100 \mathrm{~mL})$. The combined organic phases were dried over $\mathrm{MgSO}_{4}$, filtered and evaporated under reduced pressure to give a colorless oil. This oil was purified by column chromatography (hexanes/ethyl acetate 3:1) to afford triacetate 38 (950 mg, 97\%) as a colorless oil. $\mathrm{R}_{f}=0.55$ (eluent hexanes/ethyl acetate $\left.4: 1\right) ;[\boldsymbol{\alpha}]^{\mathbf{2 0}}{ }_{\mathbf{D}}+11.8\left(\mathrm{c} 0.5, \mathrm{CH}_{2} \mathrm{Cl}_{2}\right) ;{ }^{1} \mathbf{H} \mathbf{~ N M R}(500$ $\left.\mathrm{MHz}, \mathrm{CDCl}_{3}\right) \delta 6.03(\mathrm{dd}, J=9.0$ and $2.4 \mathrm{~Hz}, 1 \mathrm{H}), 5.55(\mathrm{dt}, J=4.2$ and $3.2 \mathrm{~Hz}, 1 \mathrm{H}), 5.13(\mathrm{dd}, J$ $=9.0$ and $3.2 \mathrm{~Hz}, 1 \mathrm{H}), 4.19(\mathrm{dd}, J=9.3$ and $1.5 \mathrm{~Hz}, 1 \mathrm{H}), 4.09(\mathrm{~m}, 2 \mathrm{H}), 3.73(\mathrm{~m}, 2 \mathrm{H}), 2.12(\mathrm{~s}$, $3 \mathrm{H}), 2.09$ (s, $3 \mathrm{H}), 2.08$ (m, $1 \mathrm{H}), 2.02$ (s, $3 \mathrm{H}), 1.98$ (m, $1 \mathrm{H}), 1.38$ (s, $3 \mathrm{H}), 1.36$ (s, $3 \mathrm{H}), 0.92$ (s, $9 \mathrm{H}), 0.09$ (s, $3 \mathrm{H}), 0.08$ (s, $3 \mathrm{H}) ;{ }^{13} \mathbf{C}$ NMR (125 MHz, $\left.\mathrm{CDCl}_{3}\right) \delta$ 169.8, 169.5, 169.0, 109.7, 90.6, 78.8, 78.2, 73.0, 67.8, 67.0, 64.1, 34.2, 33.6, 26.9, 25.9 (3 C), 20.9, 20.9, 20.7, 18.3, -5.3, -5.4; IR $v_{\max } / \mathrm{cm}^{-1} 2934,1749,1370,1221,1065,838 ;$ MS m/z (ES) $527(\mathrm{M}+\mathrm{Na})^{+}$; HRMS (ES): $m / z$ calcd for $\mathrm{C}_{23} \mathrm{H}_{40} \mathrm{O}_{10} \mathrm{SiNa}$ : 527.2288 found 527.2274.

$(2 R, 3 R, 4 R, 6 S)-\quad$ and $\quad(2 S, 3 R, 4 R, 6 S)-3-($ Acetyloxy)-2-[(4S,5R)-5-([1-(tert-butyl)-1,1dimethylsilyl]oxymethyl)-2,2-dimethyl-1,3-dioxolan-4-yl]-6-(phenylsulfanyl)tetrahydro2H-4-pyranyl acetate (39eq and 39ax)
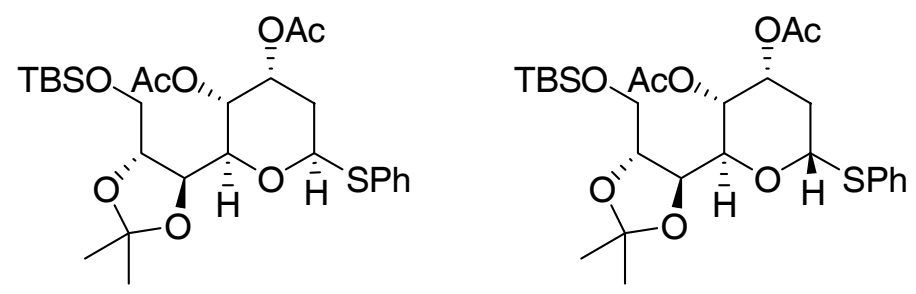

Phenylthiotrimethylsilane $(1.73 \mathrm{~mL}, 9.20 \mathrm{mmol})$ was added to a solution of acetoxy ether 38 (928 mg, $1.84 \mathrm{mmol})$ at rt. $n$-Tetrabutylammonium iodide $(815 \mathrm{mg}, 2.21 \mathrm{mmol})$ was added and the reaction mixture was cooled to $0{ }^{\circ} \mathrm{C}$. $\mathrm{ZnI}_{2}(1.76 \mathrm{~g}, 5.52 \mathrm{mmol})$ was then added and the 
resultant mixture was stirred at $0{ }^{\circ} \mathrm{C}$ until no starting material was detected by ES analysis of aliquots (normally $2 \mathrm{~h}$ ). The reaction mixture was diluted with DCM (50 mL) and treated with $0.5 \mathrm{~N} \mathrm{NaOH}(15 \mathrm{~mL})$. The organic phase was separated and washed with brine $(20 \mathrm{~mL})$. The organic phase was then dried over $\mathrm{MgSO}_{4}$, filtered and evaporated under reduced pressure to give the crude product, which was purified by column chromatography (hexanes/ethyl acetate 8:1) to afford phenylthio acetals $39(1.01 \mathrm{~g}, 98 \%)$ as an inseparable mixture of isomers. These compounds were characterized as a 1:1 mixture of isomers as follows: $\mathrm{R}_{f}=0.3$ (eluent hexanes/ethyl acetate 8:1); ${ }^{1} \mathbf{H}$ NMR $\left(500 \mathrm{MHz}, \mathrm{CDCl}_{3}\right) \delta 7.50(\mathrm{~m}, 2 \mathrm{H}), 7.50(\mathrm{~m}, 2 \mathrm{H}), 7.33(\mathrm{~m}$, $3 \mathrm{H}), 7.24-7.33(\mathrm{~m}, 3 \mathrm{H}), 5.54(\mathrm{dd}, J=6.1$ and $2.7 \mathrm{~Hz}, 1 \mathrm{H}), 5.47$ (q, $J=3.2 \mathrm{~Hz}, 1 \mathrm{H}), 5.44(\mathrm{~m}$, $1 \mathrm{H}), 5.15(\mathrm{dd}, J=9.3$ and $3.2 \mathrm{~Hz}, 1 \mathrm{H}) 5.13(\mathrm{dd}, J=12.3$ and $8.7 \mathrm{~Hz}, 1 \mathrm{H}), 4.95(\mathrm{dd}, J=8.3$ and $3.2 \mathrm{~Hz}, 1 \mathrm{H}), 4.70(\mathrm{dd}, J=9$ and $3 \mathrm{~Hz}, 4 \mathrm{H}), 4.07-4.18$ (m, $4 \mathrm{H}), 3.66-3.77$ (m, $5 \mathrm{H}), 2.44$ (ddd, $J=15.0,6.1$ and $3.2 \mathrm{~Hz}, 1 \mathrm{H}), 2.30(\mathrm{ddd}, J=15.0,4.9$ and $2.7 \mathrm{~Hz}, 1 \mathrm{H}), 2.18$ (s, $3 \mathrm{H}), 2.16$ (ddd, $J=14.4,3.7$ and $2.5 \mathrm{~Hz}, 1 \mathrm{H}), 2.11(\mathrm{~s}, 3 \mathrm{H}), 2.06(\mathrm{~m}, 3 \mathrm{H}), 2.04(\mathrm{~m}, 1 \mathrm{H}), 1.99(\mathrm{~s}, 3 \mathrm{H}), 1.38(\mathrm{~s}$, $6 \mathrm{H}), 1.37$ (s, $3 \mathrm{H}), 1.35$ (s, $3 \mathrm{H}), 0.89$ (s, $9 \mathrm{H}), 0.88$ (s, $9 \mathrm{H}), 0.07$ (s, $3 \mathrm{H}), 0.06$ (s, $9 \mathrm{H}), 0.05$ (s, $3 \mathrm{H}), 0.04$ (s, $3 \mathrm{H}) ;{ }^{13} \mathrm{C}$ NMR $\left(125 \mathrm{MHz}, \mathrm{CDCl}_{3}\right) \delta$ 170.1, 169.8, 169.6, 169.4, 136.2, 133.4, 131.6, 131.0, 128.8 (2 C), 128.8 (2 C), 127.5 (2 C), 127.1 (2 C), 109.4, 109.3, 82.9, 80.3, 78.6, 78.1, 77.8, 77.6, 74.0, 68.2, 68.0, 67.8, 67.6, 66.8, 63.7, 63.7, 35.8, 34.3, 27.0, 27.0, 26.9, 26.9, 25.9 (3 C), 25.9 (3 C), 21.2, 20.9, 20.8, 20.7, 18.3, 18.3, -5.3, -5.3, -5.4, -5.4; MS m/z (ES) 577 $(\mathrm{M}+\mathrm{Na})^{+} ;$HRMS (ES): $\mathrm{m} / z$ calcd for $\mathrm{C}_{25} \mathrm{H}_{42} \mathrm{O}_{8} \mathrm{SSiNa}$ : 577.2267 found 527.2260.

$(2 R, 3 R, 4 R, 6 R)-2-[(4 S, 5 R)-5-\quad$ and $(2 R, 3 R, 4 R, 6 S)-2-[(4 S, 5 R)-5-([1-(t e r t-B u t y l)-1,1-$ dimethylsilyl]oxymethyl)-2,2-dimethyl-1,3-dioxolan-4-yl]-6-(phenylsulfanyl)tetrahydro2H-3,4-pyrandiol (21eq and 21ax)
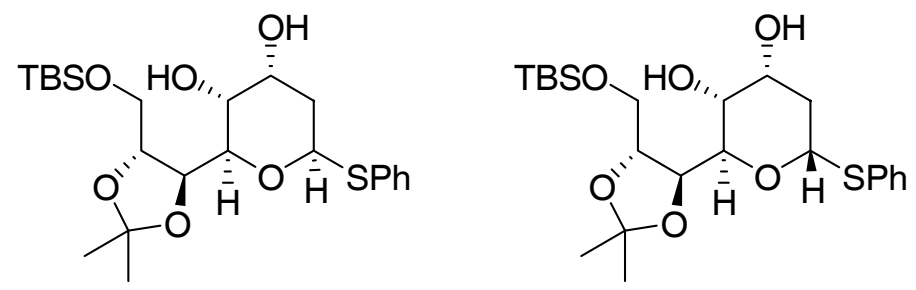

$\mathrm{K}_{2} \mathrm{CO}_{3}(748 \mathrm{mg}, 5.41 \mathrm{mmol})$ was added portionwise to a solution of a 1:1 mixture of acetates 39 ( $1 \mathrm{~g}, 1.80 \mathrm{mmol})$ in $\mathrm{MeOH}$ at $0{ }^{\circ} \mathrm{C}$. The resultant mixture was stirred at $\mathrm{rt}$ for $2.5 \mathrm{~h}$. The reaction was quenched by addition of water and then extracted with ethyl acetate $(2 \times 100$ $\mathrm{mL}$ ). The combined organic extracts were dried over $\mathrm{MgSO}_{4}$, filtered and evaporated under reduced pressure to give a wet oil. This oil was redisolved in ethyl acetate $(100 \mathrm{~mL})$ and washed with sat. aq. $\mathrm{NH}_{4} \mathrm{Cl}(50 \mathrm{~mL})$. The combined organic extracts were dried over $\mathrm{MgSO}_{4}$, filtered 
and evaporated under reduced pressure to give a residue. The crude material was purified by column chromatography (hexanes/ether 1:1) to afford a mixture of phenylthio acetals 21 (780 $\mathrm{mg}, 92 \%)$ as an inseparable mixture of isomers. This compound was characterized as a 1:1 mixture of isomers as follows: $\mathrm{R}_{f}=0.3$ (eluent hexanes/ethyl ether 1:1); ${ }^{1} \mathbf{H}$ NMR (500 MHz, $\left.\mathrm{CDCl}_{3}\right) \delta 7.33(\mathrm{~m}, 2 \mathrm{H}), 7.32(\mathrm{~m}, 2 \mathrm{H}), 7.10-7.20(\mathrm{~m}, 3 \mathrm{H}), 7.20(\mathrm{~m}, 3 \mathrm{H}), 5.45(\mathrm{~d}, J=6.1 \mathrm{~Hz}, 1$ $\mathrm{H}), 5.13(\mathrm{dd}, J=11.8$ and $2.0 \mathrm{~Hz}, 1 \mathrm{H}), 4.18(\mathrm{t}, J=8.8 \mathrm{~Hz}, 1 \mathrm{H}), 4.07(\mathrm{q}, J=3.2 \mathrm{~Hz}, 1 \mathrm{H}), 4.04$ (m, $1 \mathrm{H}), 3.97$ (t, $J=7.6 \mathrm{~Hz}, 1 \mathrm{H}), 3.85-3.82(\mathrm{~m}, 2 \mathrm{H}), 3.81-3.84(\mathrm{~m}, 2 \mathrm{H}), 3.72$ (bs, $1 \mathrm{H}), 3.68$ $(\mathrm{dd}, J=11.5$ and $2.2 \mathrm{~Hz}, 1 \mathrm{H}), 3.62(\mathrm{~m}, 1 \mathrm{H}), 3.61(\mathrm{~m}, 1 \mathrm{H}), 3.53-3.58(\mathrm{~m}, 2 \mathrm{H}), 3.43(\mathrm{dd}, J=$ 11.5 and $4.0 \mathrm{~Hz}, 1 \mathrm{H}), 3.36$ (dd, $J=11.3$ and $3.7 \mathrm{~Hz}, 1 \mathrm{H}$ ), 2.79 (bs, $1 \mathrm{H}), 2.66$ (bs, $1 \mathrm{H}), 2.39$ $(\mathrm{dd}, J=15.0$ and $0.9 \mathrm{~Hz}, 1 \mathrm{H}), 2.22(\mathrm{ddd}, J=15.0,6.3$ and $2.9 \mathrm{~Hz}, 1 \mathrm{H}), 2.12(\mathrm{ddd}, J=14.2,3.4$ and $2.2 \mathrm{~Hz}, 1 \mathrm{H}), 1.77$ (ddd, $J=14.4,3.4$ and $2.7 \mathrm{~Hz}, 1 \mathrm{H}), 1.33(\mathrm{~s}, 9 \mathrm{H}), 1.30$ (s, $3 \mathrm{H}), 0.80$ (s, 9 H), 0.79 (s, $9 \mathrm{H}),-0.05$ (s, $3 \mathrm{H}),-0.04(\mathrm{~s}, 3 \mathrm{H}),-0.03$ (s, $3 \mathrm{H}),-0.02$ (s, $3 \mathrm{H}) ;{ }^{13} \mathbf{C}$ NMR (125 $\left.\mathrm{MHz}, \mathrm{CDCl}_{3}\right) \delta 137.0,133.5,131.5$ (2 C), 130.1 (2 C), 128.8 (2 C), 128.7 (2 C), 128.2 (2 C), 127.3, 126.6, 110.3, 110.0, 82.5, 82.1, 82.0, 79.3, 77.7, 77.6, 75.9, 71.5, 71.5, 67.9, 66.6, 65.3, 63.0, 62.5, 36.1, 35.4, 27.1, 27.0, 26.9, 26.9, 25.9 (3 C), 25.9 (3 C), 18.4, 18.3, -5.2, -5.2, -5.4, -5.5; MS m/z (ES) $470(\mathrm{M}+\mathrm{Na})^{+}$; HRMS (ES): $\mathrm{m} / z$ calcd for $\mathrm{C}_{23} \mathrm{H}_{38} \mathrm{O}_{6} \mathrm{SSiNa}: 493.2056$ found 493.2039.

tert-Butyl[(4R,5S)-2,2-dimethyl-5-( $(2 R, 3 R, 4 R, 6 R)-6-$ and tert-Butyl[$[(4 R, 5 S)-2,2-d i m e t h y l-5-$ $((2 R, 3 R, 4 R, 6 S)-6-($ phenylsulfanyl)-3,4-di[2-(1,1,1-

trimethylsilyl)ethoxy]methoxytetrahydro-2H-2-pyranyl)-1,3-dioxolan-4-

yl]methoxydimethylsilane (22eq and 22ax)
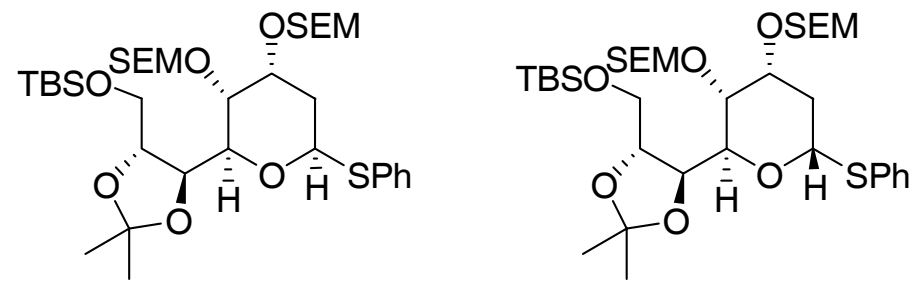

A suspension of $\mathrm{KH}$ (30\% in mineral oil, $305 \mathrm{mg}, 2.28 \mathrm{mmol}$ ) was placed in a flask under argon. The residue was washed with anhydrous hexanes $(2 \times 10 \mathrm{~mL})$ under argon. The last traces of hexanes were removed by a stream of argon. The reaction flask was cooled to $0{ }^{\circ} \mathrm{C}$. A solution of a 1:1 mixture of diols $21(214 \mathrm{mg}, 0.45 \mathrm{mmol})$ in anhydrous THF was added to the reaction flask at $0{ }^{\circ} \mathrm{C}$. After stirring at $\mathrm{rt}$ for $15 \mathrm{~min}$, SEMCl $(322 \mu \mathrm{L}, 1.82 \mathrm{mmol})$ was then added dropwise. The resultant mixture was stirred at $\mathrm{rt}$ for 3 days, and the reaction quenched by sequential addition of sat. aq. $\mathrm{NaHCO}_{3}(10 \mathrm{~mL})$ and ethyl acetate $(5 \mathrm{~mL})$. The organic phase was 
separated and the aqueous layer was extracted with ethyl acetate $(2 \times 30 \mathrm{~mL})$. The combined organic extracts were dried over $\mathrm{MgSO}_{4}$, filtered and evaporated under reduced pressure to give a residue. The crude oil was purified by column chromatography (hexanes/ether 8:1) to afford phenylthio acetals 22eq (88 $\mathrm{mg}, 26 \%$ ) and 22ax (88 $\mathrm{mg}, 26 \%)$.

22eq; colorless oil; $\mathrm{R}_{f}=0.25$ (eluent hexanes/ether 6:1); $[\alpha]^{20}{ }_{\mathrm{D}}+48.18\left(\mathrm{c} 0.55, \mathrm{CH}_{2} \mathrm{Cl}_{2}\right)$; ${ }^{1} \mathbf{H}$ NMR $\left(500 \mathrm{MHz}, \mathrm{CDCl}_{3}\right) \delta 7.48(\mathrm{~m}, 2 \mathrm{H}), 7.20-7.29(\mathrm{~m}, 3 \mathrm{H}) 5.21(\mathrm{dd}, J=11.5$ and $1.7 \mathrm{~Hz}$, $1 \mathrm{H}), 4.81(\mathrm{~m}, 2 \mathrm{H}), 4.76(\mathrm{~m}, 2 \mathrm{H}), 4.19(\mathrm{~m}, 1 \mathrm{H}), 4.13-4.17(\mathrm{~m}, 2 \mathrm{H}), 4.03(\mathrm{dd}, J=9.8$ and 3.2 $\mathrm{Hz}, 1 \mathrm{H}), 3.78(\mathrm{dd}, J=11.5$ and $4.0 \mathrm{~Hz}, 1 \mathrm{H}), 3.75(\mathrm{~m}, 1 \mathrm{H}), 3.68-3.72(\mathrm{~m}, 2 \mathrm{H}), 3.64(\mathrm{dd}, J=$ 9.8 and $3.0 \mathrm{~Hz}, 1 \mathrm{H}), 3.52-3.58(\mathrm{~m}, 2 \mathrm{H}), 2.32$ (ddd, $J=14.0,12.0$ and $2.5 \mathrm{~Hz}, 1 \mathrm{H}$ ), 1.87 (ddd, $J=14.0,12.0$ ad $2.5 \mathrm{~Hz}, 1 \mathrm{H}), 1.39$ (s, $3 \mathrm{H}), 1.37$ (s, $3 \mathrm{H}), 0.85-0.98$ (m, $4 \mathrm{H}), 0.89$ (s, $9 \mathrm{H})$, 0.07 (s, $3 \mathrm{H}), 0.06$ (s, $3 \mathrm{H}), 0.02$ (s, $9 \mathrm{H}),-0.02$ (s, $9 \mathrm{H}) ;{ }^{13} \mathbf{C ~ N M R}\left(125 \mathrm{MHz}, \mathrm{CDCl}_{3}\right) \delta 134.5$, 130.9 (2 C), 128.7 (2 C), 126.9, 108.7, 95.3, 95.0, 80.1, 78.0, 77.9, 75.6, 75.0, 73.9, 65.7, 65.4, 63.6, 37.1, 34.2, 27.0, 26.9, 25.9 (3 C), 18.3, 18.1, -1.4 (3 C), -1.4 (3 C), -5.2, -5.3; IR $v_{\max } / \mathrm{cm}^{-1} 2953,1369,1250,1063,836 ;$ MS m/z (ES) $753(\mathrm{M}+\mathrm{Na})^{+}$; HRMS (ES): $\mathrm{m} / \mathrm{z}$ calcd for $\mathrm{C}_{35} \mathrm{H}_{66} \mathrm{O}_{8} \mathrm{SSi}_{3} \mathrm{Na}$ : 753.3684, found 753.3686.

22ax; colorless oil; $\mathrm{R}_{f}=0.15$ (eluent hexanes/ether 6:1); $[\boldsymbol{\alpha}]_{\mathbf{D}}=+117.3$ (c 1.05, $\left.\mathrm{CH}_{2} \mathrm{Cl}_{2}\right) ;{ }^{1} \mathbf{H}$ NMR $\left(500 \mathrm{MHz}, \mathrm{CDCl}_{3}\right) \delta 7.48(\mathrm{~m}, 2 \mathrm{H}), 7.27-7.19(\mathrm{~m}, 3 \mathrm{H}) 5.45(\mathrm{dd}, J=5.6$ and $3.9 \mathrm{~Hz}, 1 \mathrm{H}), 4.79-4.85(\mathrm{~m}, 4 \mathrm{H}), 4.51(\mathrm{dd}, J=7.8$ and $3.4 \mathrm{~Hz}, 1 \mathrm{H}), 4.11-4.20(\mathrm{~m}, 3 \mathrm{H}), 3.84-$ 3.89 (m, 2 H), 3.60-3.78 (m, 5 H), 2.21-2.32 (m, 2 H), 1.42 (s, 3 H), 1.38 (s, 3 H), 0.88-0.98 (m, $4 \mathrm{H}), 0.88$ (s, $9 \mathrm{H}), 0.04$ (s, $9 \mathrm{H}), 0.03$ (s, $3 \mathrm{H}), 0.02$ (s, $9 \mathrm{H}), 0.01$ (s, $3 \mathrm{H}) ;{ }^{13} \mathbf{C} \mathbf{N M R}(125$ $\left.\mathrm{MHz}, \mathrm{CDCl}_{3}\right) \delta 136.9,130.9$ (2 C), 128.6 (2 C), 126.7, 108.9, 94.9, 94.4, 82.8, 78.5, 77.8, 74.1, 71.9, 70.0, 65.7, 65.6, 63.1, 34.8, 29.6, 27.1, 26.8, 25.9 (3C), 18.3, 18.1, -1.3 (3 C), -1.3 (3 C), 5.3, -5.4; IR $v_{\max } / \mathrm{cm}^{-1} 2953,1472,1250,1064,1025,836 ; \mathbf{M S ~ m / z ~ ( E S ) ~} 445(\mathrm{M}+\mathrm{Na})^{+}$; HRMS (ES): $m / z$ calcd for $\mathrm{C}_{35} \mathrm{H}_{66} \mathrm{O}_{8} \mathrm{SSi}_{3} \mathrm{Na}$ : 753.3684, found 753.3705. 


\section{Reductive Lithiation of Phenylthio Acetals 20, 21 and 22}
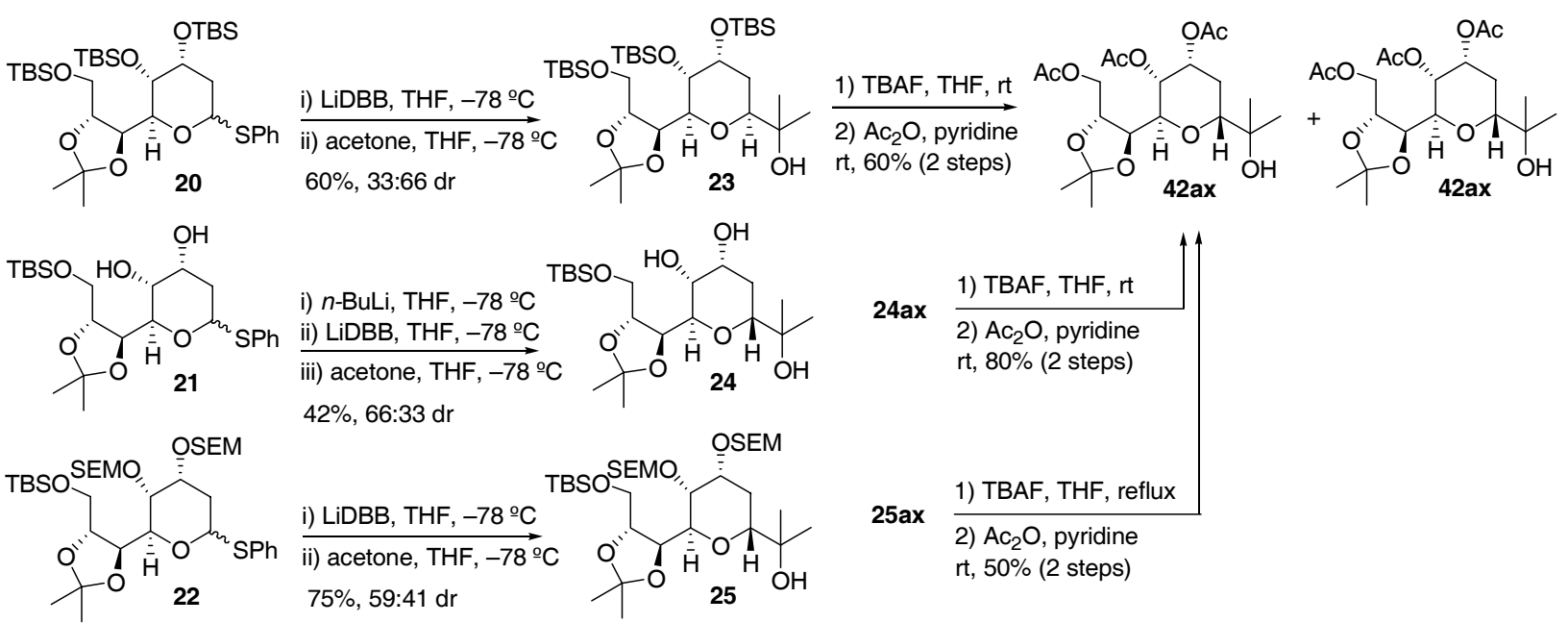

2-(2S,4R,5S,6S)-4,5- and 2-(2R,4R,5S,6S)-4,5-Di[1-(tert-butyl)-1,1-dimethylsilyl]oxy-6[(4R,5R)-5-([1-(tert-butyl)-1,1-dimethylsilyl]oxymethyl)-2,2-dimethyl-1,3-dioxolan-4yl]tetrahydro-2H-2-pyranyl-2-propanol (23eq and 23ax)

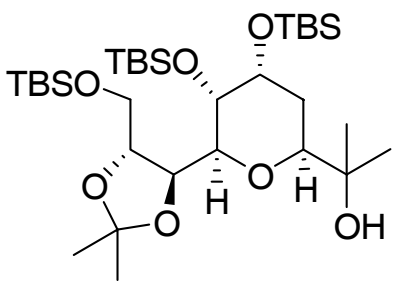

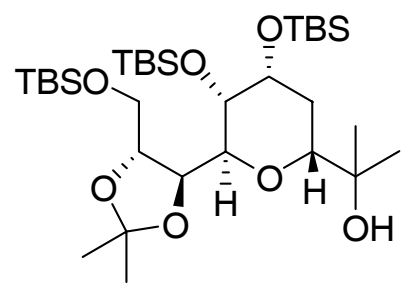

According to the reductive lithiation procedure described in section 2, treatment of a 1:1 mixture of phenylthio acetals 20 (125 $\mathrm{mg}, 0.179 \mathrm{mmol})$ with LiDBB followed by reaction with acetone $(53 \mu \mathrm{L}, 0.716 \mathrm{mmol})$ and chromatography (eluent hexanes/ether 8:1) afforded alcohols $23(68 \mathrm{mg}, 60 \%)$ as a 2:1 mixture of diastereomers in favour of alcohol 23eq. Protonated adduct 40 (31 mg, 30\%) was also isolated. The complex mixture of isomers 23 was further elaborated to triacetates $\mathbf{4 2}$ and $\mathbf{4 3}$ for stereochemical analysis and characterization purposes (vide infra).

23ax; Characterized from an enriched mixture of both diastereomers. colorless oil; $\mathrm{R}_{f}=$ 0.4 (eluent hexanes/ether 4:1); ${ }^{1} \mathrm{H}$ NMR $\left(500 \mathrm{MHz}, \mathrm{CDCl}_{3}\right) \delta 4.25$ (m, $\left.1 \mathrm{H}\right), 4.19$ (ddd, $J=11.0$, 5.1 and $3.4 \mathrm{~Hz}, 1 \mathrm{H}), 3.98(\mathrm{bd}, J=3.0 \mathrm{~Hz}, 1 \mathrm{H}), 3.82(\mathrm{~m}, 2 \mathrm{H}), 3.72(\mathrm{~m}, 2 \mathrm{H}), 3.62(\mathrm{dd}, J=13.2$ and $1.7 \mathrm{~Hz}, 1 \mathrm{H}), 2.70(\mathrm{~s}, 3 \mathrm{H}), 1.90(\mathrm{td}, J=13.0$ and $11.3 \mathrm{~Hz}, 1 \mathrm{H}), 1.25(\mathrm{~s}, 3 \mathrm{H}), 0.94(\mathrm{~s}, 9 \mathrm{H})$, 0.92 (s, 9 H), 0.91 (s, 9 H), 0.11 (s, $6 \mathrm{H}), 0.08$ (s, 3 H), 0.07 (s, $3 \mathrm{H}), 0.03$ (s, $3 \mathrm{H}),-0.05$ (s, 3 $\mathrm{H})$. 
23eq; Characterized from an enriched mixture of both diastereomers. colorless oil; $\mathrm{R}_{f}=$ 0.3 (eluent hexanes/ether 4:1); ${ }^{1} \mathbf{H}$ NMR $\left(500 \mathrm{MHz}, \mathrm{C}_{6} \mathrm{D}_{6}\right.$, only representative signals reported) $\delta 4.23$ (bs, $1 \mathrm{H}), 4.20$ (t, $J=7.3 \mathrm{~Hz}, 1 \mathrm{H}), 3.80$ (m, $1 \mathrm{H}$ ), 2.06 (bs, $1 \mathrm{H}), 1.71$ (bs, $1 \mathrm{H}), 1.44$ (s, 3 H), 1.39 (s, 3 H), 1.20 (s, 3 H), 1.14 (s, 3 H), 1.06 (s, 9 H), 1.01 (s, 9 H), 0.99 (s, 9 H), 0.22 (s, 3 H), 0.17 (s, $3 \mathrm{H}), 0.14$ (s, $6 \mathrm{H}), 0.10$ (s, $3 \mathrm{H}) . \mathrm{MS}$ m/z (ES) $648(\mathrm{M}+\mathrm{Na})^{+}$; HRMS (ES): $m / z$ calcd for $\mathrm{C}_{32} \mathrm{H}_{68} \mathrm{O}_{7} \mathrm{Si}_{3} \mathrm{Na}$ : 671.4171, found 671.4178 .

tert-Butyl[(4R,5R)-5-((2S,3S,4R)-3,4-di[1-(tert-butyl)-1,1-dimethylsilyl]oxytetrahydro-2H-2pyranyl)-2,2-dimethyl-1,3-dioxolan-4-yl]methoxydimethylsilane (40)

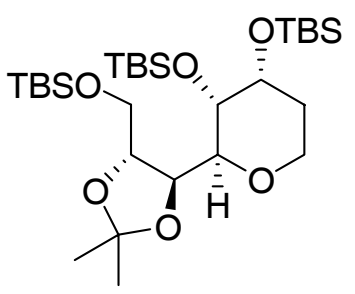

40; colorless oil; $\mathrm{R}_{f}=0.5$ (eluent hexanes/ether 4:1); $[\alpha]^{20}{ }_{\mathrm{D}}+9.6\left(\mathrm{c} 0.6, \mathrm{CH}_{2} \mathrm{Cl}_{2}\right)$; ${ }^{\mathbf{1}} \mathbf{H}$ NMR (500 MHz, C $\left.6 \mathrm{D}_{6}\right) \delta 4.37(\mathrm{~m}, 1 \mathrm{H}), 4.34$ (d, $\left.J=8.1 \mathrm{~Hz}, 1 \mathrm{H}\right), 4.00-4.08$ (m, $\left.2 \mathrm{H}\right), 3.95$ (dd, $J=8.1$ and $1.1 \mathrm{~Hz}, 1 \mathrm{H}), 3.87(\mathrm{dd}, J=11.4$ and $2.7 \mathrm{~Hz}, 1 \mathrm{H}), 3.79(\mathrm{dd}, J=11.4$ and $4.2 \mathrm{~Hz}, 1$ H), $3.62(\mathrm{dd}, J=13.5$ and $2.2 \mathrm{~Hz}, 1 \mathrm{H}), 2.10(\mathrm{q}, J=12.2 \mathrm{~Hz}, 1 \mathrm{H}), 1.88(\mathrm{dd}, J=10.2 \mathrm{~Hz}, 1 \mathrm{H})$, 1.64 (ddd, $J=12.2,4.2$ and $2.2 \mathrm{~Hz}, 1 \mathrm{H}), 1.42$ (s, $3 \mathrm{H}), 1.34$ (s, $3 \mathrm{H}), 0.99$ (s, $27 \mathrm{H}), 0.15$ (s, 3 H), 0.14 (s, 3 H), 0.13 (s, 3 H), 0.12 (s, 3 H), 0.08 (s, 3 H), 0.05 (s, 3 H); ${ }^{13}$ C NMR (125 MHz, $\left.\mathrm{C}_{6} \mathrm{D}_{6}\right) \delta 109.4,82.4,82.3,73.9,70.2,66.6,64.6,63.4,31.9,30.1,27.4,27.3$ (3 C), 26.1 (3 C), 25.9 (3 C), 18.5, 18.2, 17.1, -4.5, -4.5, -5.0, -5.0, -7.5, -7.7; IR $v_{\max } / \mathrm{cm}^{-1} 2930,1472,1254$, 1129, 838, 777; MS m/z (ES) $613(\mathrm{M}+\mathrm{Na})^{+}$; HRMS (ES): $m / z$ calcd for $\mathrm{C}_{29} \mathrm{H}_{62} \mathrm{O}_{6} \mathrm{Si}_{3} \mathrm{Na}$ : 613.3752, found 613.3762 .

2-((2S,4R,5S,6R)-6-[(4R,5R)- $\quad$ and $\quad 5([1-(t e r t-B u t y l)-1,1-d i m e t h y l s i l y l]$ oxymethyl)-2,2dimethyl-1,3-dioxolan-4-yl]-4,5-di[2-(1,1,1-trimethylsilyl)ethoxy]methoxytetrahydro-2H-2pyranyl)-2-propanol (25eq and 25ax)
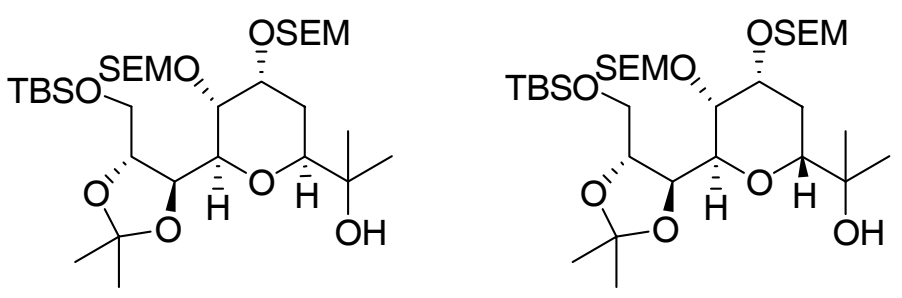
According to the reductive lithiation procedure described in section 2, treatment of a 1:1 mixture of phenylthio acetals $22(115 \mathrm{mg}, 0.157 \mathrm{mmol})$ with LiDBB followed by reaction with acetone $(58 \mu \mathrm{L}, 0.785 \mathrm{mmol})$ and chromatography (eluent hexanes/ether 4:1) afforded alcohol 25ax (64 mg, 50\%) and alcohol 25eq (44 mg, 35\%).

25eq; colorless oil; $\mathrm{R}_{f}=0.2$ (eluent hexanes/ether 2:1); [ $\left.\mathbf{\alpha}\right]^{\mathbf{2 0}}{ }_{\mathbf{D}}-13.93\left(\mathrm{c} 1.4, \mathrm{CH}_{2} \mathrm{Cl}_{2}\right.$ ); ${ }^{\mathbf{1}} \mathbf{H}$ NMR $\left(500 \mathrm{MHz}, \mathrm{CDCl}_{3}\right) \delta 4.74-4.86(\mathrm{~m}, 4 \mathrm{H}), 4.21(\mathrm{dt}, J=8.3$ and $4.2 \mathrm{~Hz}, 1 \mathrm{H}), 4.12-4.15(\mathrm{~m}$, $1 \mathrm{H}), 4.01(\mathrm{dd}, J=9.8$ and $2.7 \mathrm{~Hz}, 1 \mathrm{H}), 3.81(\mathrm{dd}, J=11.0$ and $3.9 \mathrm{~Hz}, 1 \mathrm{H}), 3.72-3.78(\mathrm{~m}, 3 \mathrm{H})$, 3.52-3.63 (m, $4 \mathrm{H}), 2.30(\mathrm{~s}, 1 \mathrm{H}), 2.02(\mathrm{ddd}, J=13.7,4.0$ and $2.0 \mathrm{~Hz}, 1 \mathrm{H}), 1.61$ (ddd, $J=13.7$, 11.8 and $2.2 \mathrm{~Hz}, 1 \mathrm{H}), 1.42(\mathrm{~s}, 3 \mathrm{H}), 1.38(\mathrm{~s}, 3 \mathrm{H}), 1.20$ (s, $3 \mathrm{H}), 1.14$ (s, $3 \mathrm{H}), 0.86-0.98$ (m, 4 $\mathrm{H}), 0.90(\mathrm{~s}, 18 \mathrm{H}), 0.08(\mathrm{~s}, 6 \mathrm{H}), 0.02(\mathrm{~s}, 18 \mathrm{H}) ;{ }^{13} \mathbf{C} \mathbf{N M R}\left(125 \mathrm{MHz}, \mathrm{CDCl}_{3}\right) \delta$ 108.6, 95.6, 95.1, 78.6, 78.2, 77.8, 76.2, 74.1, 74.1, 71.5, 65.7, 65.4, 63.9, 29.6, 27.1, 26.9, 26.4, 25.9 (3 C), 24.3, 18.4, 18.1, 18.1, -1.4 (3 C), -1.4 (3 C), -5.2, -5.3; IR v $v_{\max } / \mathrm{cm}^{-1} 3484,2954,1370,1250$, 1061, 860; MS m/z (ES) $703(\mathrm{M}+\mathrm{Na})^{+}$; HRMS (ES): $m / z$ calcd for $\mathrm{C}_{32} \mathrm{H}_{68} \mathrm{O}_{9} \mathrm{Si}_{3} \mathrm{Na}$ : 703.4069, found 703.4075 .

25ax; colorless oil; $\mathrm{R}_{f}=0.3$ (eluent hexanes/ether 6:1); $[\boldsymbol{\alpha}]^{\mathbf{2 0}}{ }_{\mathbf{D}}-7.26\left(\mathrm{c} 1.9, \mathrm{CH}_{2} \mathrm{Cl}_{2}\right) ;{ }^{1} \mathbf{H}$ NMR (500 MHz, $\left.\mathrm{CDCl}_{3}\right) \delta 4.73-4.82(\mathrm{~m}, 4 \mathrm{H}), 4.20(\mathrm{t}, J=7.2 \mathrm{~Hz}, 1 \mathrm{H}), 4.15$ (ddd, $J=12.3$, 4.4 and $3.2 \mathrm{~Hz}, 1 \mathrm{H}), 4.11(\mathrm{bd}, J=7.6 \mathrm{~Hz}, 1 \mathrm{H}), 4.07(\mathrm{~s}, 1 \mathrm{H}), 3.93(\mathrm{dt}, J=7.4$ and $3.7 \mathrm{~Hz}, 1 \mathrm{H})$, $3.84(\mathrm{dd}, J=11.2$ and $3.4 \mathrm{~Hz}, 1 \mathrm{H}), 3.78(\mathrm{dd}, J=11.2$ and $3.7 \mathrm{~Hz}, 1 \mathrm{H}), 3.60-3.73(\mathrm{~m}, 4 \mathrm{H})$, $3.50(\mathrm{dd}, J=12.0$ and $2.2 \mathrm{~Hz}, 1 \mathrm{H}), 2.37(\mathrm{~s}, 1 \mathrm{H}), 1.89(\mathrm{q}, J=12.0 \mathrm{~Hz}, 1 \mathrm{H}), 1.72(\mathrm{~m}, 1 \mathrm{H}), 1.40$ (s, $3 \mathrm{H}), 1.37$ (s, $3 \mathrm{H}), 1.19$ (s, $3 \mathrm{H}), 1.16$ (s, $3 \mathrm{H}), 0.86-0.97$ (m, $4 \mathrm{H}), 0.90$ (s, $9 \mathrm{H}), 0.08$ (s, 3 H), 0.07 (s, $3 \mathrm{H}), 0.03$ (s, $9 \mathrm{H}), 0.02$ (s, $9 \mathrm{H}) ;{ }^{13} \mathbf{C ~ N M R}\left(125 \mathrm{MHz}, \mathrm{CDCl}_{3}\right) \delta 109.5,94.7,92.5$, 81.2, 79.0, 77.6, 74.5, 71.8, 71.1, 70.9, 65.1, 65.0, 62.7, 29.6, 27.4, 27.1, 26.8, 26.2, 25.9 (3 C), 24.0, 18.3, 18.0, -1.4 (6 C), -5.2, -5.3; IR $v_{\max } / \mathrm{cm}^{-1} 3497,2954,1463,1372,1250,1078,859$; MS m/z (ES) $703(\mathrm{M}+\mathrm{Na})^{+}$; HRMS (ES): $\mathrm{m} / z$ calcd for $\mathrm{C}_{32} \mathrm{H}_{68} \mathrm{O}_{9} \mathrm{Si}_{3} \mathrm{Na}$ : 703.4069, found 703.4078 . 
$(2 R, 3 R, 4 R, 6 S)-2-$

and

$(2 R, 3 R, 4 R, 6 R)-2-[(4 R, 5 R)-5-([1-(t e r t-B u t y l)-1,1-$

dimethylsilyl]oxymethyl)-2,2-dimethyl-1,3-dioxolan-4-yl]-6-(1-hydroxy-1-

methylethyl)tetrahydro-2H-3,4-pyrandiol (24eq and 24ax)
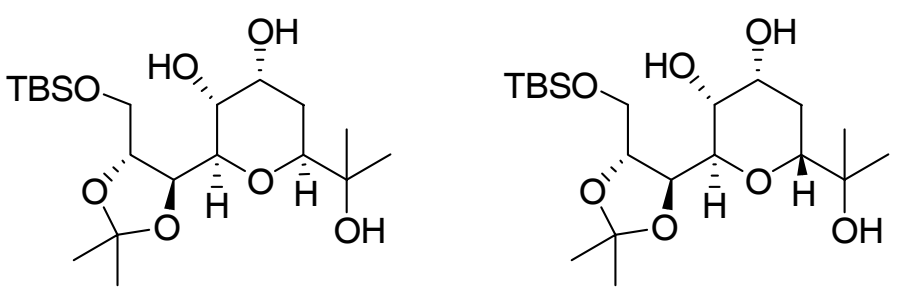

Titration of a 1:1 mixture of phenylthio acetals 21 (79 $\mathrm{mg}, 0.168 \mathrm{mmol})$ with $n$ $\mathrm{BuLi} /$ phenantroline followed by reductive lithiation using $\mathrm{LiDBB}$, addition of acetone $(100 \mu \mathrm{L}$, $1.36 \mathrm{mmol}$ ) and chromatography (eluent ether) afforded alcohol 24ax (20 mg, 29\%), alcohol 24eq (10 mg, 14\%) and protonated adduct 41 (21 mg, 30\%).

24eq; colorless oil; $\mathrm{R}_{f}=0.35$ (eluent $\mathrm{CH}_{2} \mathrm{Cl}_{2} / \mathrm{MeOH}$ 98:2); $[\boldsymbol{\alpha}]^{\mathbf{2 0}}{ }_{\mathbf{D}}+6.57$ (c 0.35 , $\left.\mathrm{CH}_{2} \mathrm{Cl}_{2}\right) ;{ }^{1} \mathbf{H}$ NMR $\left(500 \mathrm{MHz}, \mathrm{C}_{6} \mathrm{D}_{6}\right) \delta 4.05(\mathrm{~m}, 2 \mathrm{H}), 4.00(\mathrm{t}, J=8.3 \mathrm{~Hz}, 1 \mathrm{H}), 3.88(\mathrm{dd}, J=$ 11.2 and $2.5 \mathrm{~Hz}, 1 \mathrm{H}), 3.66-3.72(\mathrm{~m}, 3 \mathrm{H}), 3.46(\mathrm{dd}, J=9.3$ and $3.0 \mathrm{~Hz}, 1 \mathrm{H}), 1.86(\mathrm{ddd}, J=$ 14.0, 3.7 and $2.2 \mathrm{~Hz}, 1 \mathrm{H}), 1.36(\mathrm{~m}, 1 \mathrm{H}), 1.32(\mathrm{~s}, 3 \mathrm{H}), 1.22(\mathrm{~s}, 3 \mathrm{H}), 1.12(\mathrm{~s}, 3 \mathrm{H}), 1.04(\mathrm{~s}, 3 \mathrm{H})$, 0.98 (s, $9 \mathrm{H}), 0.10$ (s, $6 \mathrm{H}) ;{ }^{13} \mathbf{C} \mathbf{N M R}\left(125 \mathrm{MHz}, \mathrm{CDCl}_{3}\right) \delta 110.2,82.3,78.5,77.3,75.3,72.2$, 71.5, 66.2, 63.3, 30.3, 27.1, 27.0, 26.6, 25.9 (3C), 24.4, 18.4, -5.2, -5.3; IR $v_{\max } / \mathrm{cm}^{-1} 3444$, 2929, 1372, 1253, 1088, 837; MS m/z (ES) $443(\mathrm{M}+\mathrm{Na})^{+}$; HRMS (ES): $m / z$ calcd for $\mathrm{C}_{20} \mathrm{H}_{40} \mathrm{O}_{7} \mathrm{SiNa}$ : 443.2441, found 443.2423.

24ax; colorless oil; $\mathrm{R}_{f}=0.2$ (eluent ether); $[\boldsymbol{\alpha}]^{\mathbf{2 0}}{ }_{\mathbf{D}}-1.77$ (c $\left.0.45, \mathrm{CH}_{2} \mathrm{Cl}_{2}\right) ;{ }^{1} \mathbf{H}$ NMR (500 $\left.\mathrm{MHz}, \mathrm{CDCl}_{3}\right) \delta 4.10-4.16(\mathrm{~m}, 3 \mathrm{H}), 4.01(\mathrm{dt}, J=6.8$ and $3.5 \mathrm{~Hz}, 1 \mathrm{H}), 3.90(\mathrm{t}, J=3.3 \mathrm{~Hz}, 1 \mathrm{H})$, $3.87(\mathrm{dd}, J=8.0$ and $4.7 \mathrm{~Hz}, 1 \mathrm{H}), 3.80(\mathrm{dd}, J=11.1$ and $3.7 \mathrm{~Hz}, 1 \mathrm{H}), 3.52(\mathrm{dd}, J=8.0$ and 4.7 Hz, $1 \mathrm{H}), 3.05$ (bs, $1 \mathrm{H}), 2.95$ (bs, $1 \mathrm{H}), 2.77$ (bs, $1 \mathrm{H}), 1.82-1.90$ (m, $2 \mathrm{H}), 1.40$ (s, $3 \mathrm{H}), 1.24$ (s, $6 \mathrm{H}), 1.16(\mathrm{~s}, 3 \mathrm{H}), 0.90(\mathrm{~s}, 9 \mathrm{H}), 0.08(\mathrm{~s}, 6 \mathrm{H}) ;{ }^{13} \mathbf{C ~ N M R}\left(125 \mathrm{MHz}, \mathrm{CDCl}_{3}\right) \delta$ 109.6, 81.1, 77.2 76.9, 76.1, 72.2, 68.6, 65.9, 62.7, 28.5, 27.2, 27.0, 26.6, 25.9 (3C), 25.5, 18.3, -5.2, -5.3; IR $v_{\max } / \mathrm{cm}^{-1} 3394,2932,1381,1254,1122,837 ;$ MS m/z (ES) $443(\mathrm{M}+\mathrm{Na})^{+}$; HRMS (ES): $m / z$ calcd for $\mathrm{C}_{20} \mathrm{H}_{40} \mathrm{O}_{7} \mathrm{SiNa}$ : 443.2441, found 443.2428. 


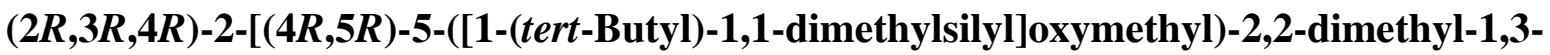
dioxolan-4-yl]tetrahydro-2H-3,4-pyrandiol (41)

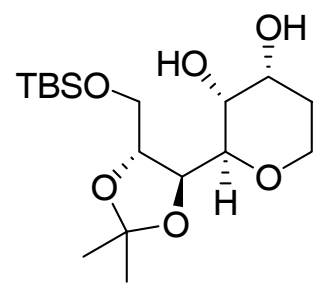

41h; colorless oil; $\mathrm{R}_{f}=0.2$ (eluent hexanes/ethyl acetate $3: 1$ ); $[\boldsymbol{\alpha}]^{\mathbf{2 0}}{ }_{\mathrm{D}}+8.79$ (c 1.9, $\left.\mathrm{CH}_{2} \mathrm{Cl}_{2}\right) ;{ }^{1} \mathbf{H}$ NMR $\left(500 \mathrm{MHz}, \mathrm{CDCl}_{3}\right) \delta 4.07-4.11(\mathrm{~m}, 2 \mathrm{H}), 3.91(\mathrm{dd}, J=11.5$ and $2.7 \mathrm{~Hz}, 1$ $\mathrm{H}), 3.84(\mathrm{dd}, J=8.6$ and $7.8 \mathrm{~Hz}, 1 \mathrm{H}), 3.83(\mathrm{~m}, 1 \mathrm{H}), 3.75(\mathrm{td}, J=11.8$ and $3.0 \mathrm{~Hz}, 1 \mathrm{H}), 3.69$ $(\mathrm{dd}, J=11.5$ and $5.1 \mathrm{~Hz}, 1 \mathrm{H}), 3.65(\mathrm{td}, J=5.1$ and $1.2 \mathrm{~Hz}, 1 \mathrm{H}), 3.61(\mathrm{dd}, J=9.3$ and $3.0 \mathrm{~Hz}, 1$ H), 3.50 (t, $J=8.8 \mathrm{~Hz}, 1 \mathrm{H}), 2.70(\mathrm{~s}, 1 \mathrm{H}), 1.78-1.89$ (m, $2 \mathrm{H}), 1.44$ (s, $3 \mathrm{H}), 1.42$ (s, $3 \mathrm{H}), 0.90$ $(\mathrm{s}, 9 \mathrm{H}), 0.08(\mathrm{~s}, 6 \mathrm{H}) ;{ }^{13} \mathbf{C} \mathbf{N M R}\left(125 \mathrm{MHz}, \mathrm{CDCl}_{3}\right) \delta$ 110.2, 82.8, 78.5, 75.5, 72.3, 65.6, 63.8, 61.6, 30.8, 29.7, 27.0, 27.0, 25.0 (3 C), 18.5, -5.2, -5.3; IR v $v_{\max } / \mathrm{cm}^{-1} 3511,2930,1372,1254$, 1094, 838; MS m/z (ES) $385(\mathrm{M}+\mathrm{Na})^{+}$; HRMS (ES): $\mathrm{m} / z$ calcd for $\mathrm{C}_{17} \mathrm{H}_{34} \mathrm{O}_{6} \mathrm{SiNa}$ : 385.2022 , found 386.2049 .

$(2 S, 3 S, 4 R, 6 S)-3-($ Acetyloxy)-2-(4R,5R)-5-[(acetyloxy)methyl]-2,2-dimethyl-1,3-dioxolan-4yl-6-(1-hydroxy-1-methylethyl)tetrahydro-2H-4-pyranyl acetate (42eq)

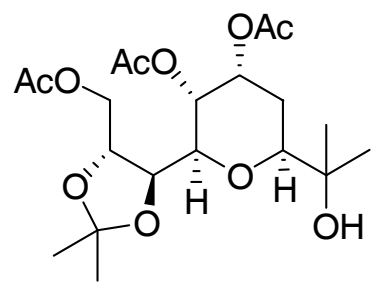

TBAF (1 $\mathrm{M}$ in THF, $558 \mu \mathrm{L}, 0.558 \mathrm{mmol}$ ) was added to a solution of 25eq (19 $\mathrm{mg}$, $0.039 \mathrm{mmol}$ ) in THF ( $3 \mathrm{~mL}$ ). After stirring at reflux for $48 \mathrm{~h}$, the solvent was evaporated under reduced pressure to give a residue. This material was dissolved in pyridine $(4 \mathrm{~mL})$ and trated with acetic anhydride $(1 \mathrm{~mL})$. After stirring at $\mathrm{rt}$ for $16 \mathrm{~h}$, ether $(3 \mathrm{~mL})$ and water $(4 \mathrm{~mL})$ were added. The organic phase was separated and the aqueous was extracted with ether $(10 \mathrm{~mL})$. The combined organic extracts were dried over $\mathrm{MgSO}_{4}$, filtered and evaporated under reduced pressure to give a pale residue. This crude material was purified by column chromatography (ether) to afford alcohol $\mathbf{4 2 e q}(6 \mathrm{mg}, 35 \%)$ as a colorless oil. $\mathrm{R}_{f}=0.6$ (eluent ether); $[\boldsymbol{\alpha}]^{\mathbf{2 0}}{ }_{\mathbf{D}}-9.5$ (c $0.2, \mathrm{CH}_{2} \mathrm{Cl}_{2}$ ); ${ }^{1} \mathrm{H}$ NMR $\left(500 \mathrm{MHz}, \mathrm{C}_{6} \mathrm{D}_{6}\right) \delta 5.39$ (q, $\left.J=3.0 \mathrm{~Hz}, 1 \mathrm{H}\right), 4.95$ (dd, $J=9.8$ and 
$3.1 \mathrm{~Hz}, 1 \mathrm{H}), 4.57(\mathrm{dd}, J=12.0$ and $2.5 \mathrm{~Hz}, 1 \mathrm{H}), 4.24(\mathrm{td}, J=7.4$ and $2.4 \mathrm{~Hz}, 1 \mathrm{H}), 3.92(\mathrm{dd}, J$ $=11.8$ and $7.5 \mathrm{~Hz}, 1 \mathrm{H}), 3.87(\mathrm{dd}, J=10.6$ and $7.3 \mathrm{~Hz}, 1 \mathrm{H}), 3.77(\mathrm{dd}, J=8.0$ and $6.6 \mathrm{~Hz}, 1 \mathrm{H})$, $3.63(\mathrm{dd}, J=11.7$ and $1.8 \mathrm{~Hz}, 1 \mathrm{H}), 3.04$ (s, $1 \mathrm{H}), 2.14$ (s, $3 \mathrm{H}), 2.11$ (s, $3 \mathrm{H}), 2.00$ (s, $3 \mathrm{H}), 1.90$ (ddd, $J=14.5,3.9$ and $2.1 \mathrm{~Hz}, 1 \mathrm{H}), 1.75$ (ddd, $J=14.5,12.0$ and $2.5 \mathrm{~Hz}, 1 \mathrm{H}), 1.40$ (s, $3 \mathrm{H}$ ), 1.37 (s, $3 \mathrm{H}), 1.16$ (s, $6 \mathrm{H}) ;{ }^{13} \mathbf{C}$ NMR $\left(125 \mathrm{MHz}, \mathrm{CDCl}_{3}\right) \delta 171.1,170.0,170.0,110.3,79.2$, 77.9, 77.6, 74.6, 71.3, 69.8, 67.6, 65.7, 30.7, 29.6, 26.9, 26.7, 25.9, 23.7, 21.1, 20.8, 20.8; IR $v_{\max } / \mathrm{cm}^{-1} 3550,2924,1746,1372,1245,1063 ;$ MS m/z (ES) $455(\mathrm{M}+\mathrm{Na})^{+} ;$HRMS (ES): $\mathrm{m} / z$ calcd for $\mathrm{C}_{20} \mathrm{H}_{32} \mathrm{O}_{10} \mathrm{Na}$ : 455.1893, found 455.1881.

Stereochemical assignment of alcohol 42eq: spin-coupling constants ${ }^{3} J_{\mathrm{H}-\mathrm{H}}$ and NOE observed

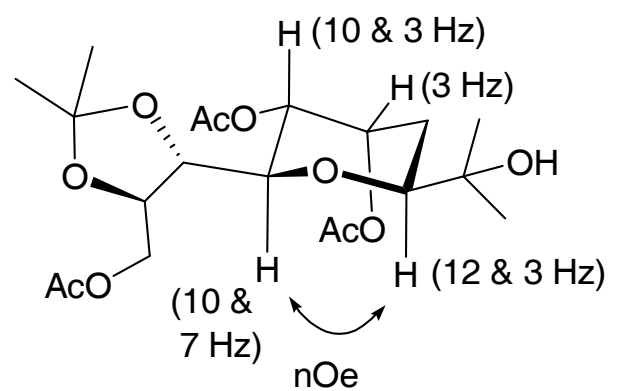

$(2 S, 3 S, 4 R, 6 R)-3-($ Acetyloxy)-2-(4R,5R)-5-[(acetyloxy)methyl]-2,2-dimethyl-1,3-dioxolan-4yl-6-(1-hydroxy-1-methylethyl)tetrahydro-2 $H$-4-pyranyl acetate (42ax)<smiles>CC(=O)OCC1OC(C)(C)O[C@H]1[C@H]1O[C@H](C(C)(C)O)C[C@@H](OC(C)=O)[C@H]1OC(C)=O</smiles>

TBAF ( $1 \mathrm{M}$ in THF, $647 \mu \mathrm{L}, 0.647 \mathrm{mmol}$ ) was added to a solution of 25ax (22 $\mathrm{mg}$, $0.032 \mathrm{mmol})$ in THF (3 mL). After stirring at reflux for $48 \mathrm{~h}$, the solvent was evaporated under reduced pressure to give a residue. This material was dissolved in pyridine $(4 \mathrm{~mL})$ and treated with acetic anhydride $(1 \mathrm{~mL})$. After stirring at $\mathrm{rt}$ for $16 \mathrm{~h}$, ether $(3 \mathrm{~mL})$ and water $(4 \mathrm{~mL})$ were added. The organic phase was separated and the aqueous was extracted with ether $(10 \mathrm{~mL})$. The combined organic extracts were dried over $\mathrm{MgSO}_{4}$, filtered and evaporated under reduced pressure to give a residue. This crude material was purified by column chromatography (ether) to afford alcohol 42ax $(7 \mathrm{mg}, 50 \%)$ as a colorless oil. $\mathrm{R}_{f}=0.35$ (eluent hexanes/ether 1:4); $[\mathbf{\alpha}]^{\mathbf{2 0}}{ }_{\mathbf{D}}$ $+24.00\left(\mathrm{c} 0.35, \mathrm{CH}_{2} \mathrm{Cl}_{2}\right) ;{ }^{1} \mathrm{H}$ NMR $\left(500 \mathrm{MHz}, \mathrm{C}_{6} \mathrm{D}_{6}\right) \delta 5.78(\mathrm{~m}, 1 \mathrm{H}), 5.55(\mathrm{ddd}, J=12.5,4.9$ 
and $3.4 \mathrm{~Hz}, 1 \mathrm{H}), 4.35(\mathrm{dd}, J=11.8$ and $3.2 \mathrm{~Hz}, 1 \mathrm{H}), 4.19$ (ddd, $J=7.1,6.4$ and $3.4 \mathrm{~Hz}, 1 \mathrm{H}$ ), 4.06-4.11 (m, $2 \mathrm{H}), 3.98(\mathrm{t}, J=7.3 \mathrm{~Hz}, 1 \mathrm{H}), 3.46(\mathrm{dd}, J=12.0$ and $2.4 \mathrm{~Hz}, 1 \mathrm{H}), 2.05(\mathrm{q}, J=$ $12.2 \mathrm{~Hz}, 1 \mathrm{H}), 1.72(\mathrm{~m}, 1 \mathrm{H}), 1.71(\mathrm{~s}, 3 \mathrm{H}), 1.70$ (s, $3 \mathrm{H}), 1.61$ (s, $3 \mathrm{H}), 1.40$ (s, $3 \mathrm{H}), 1.36$ (s, 3 $\mathrm{H}), 1.12(\mathrm{~s}, 3 \mathrm{H}), 1.11(\mathrm{~s}, 3 \mathrm{H}) ;{ }^{13} \mathbf{C} \mathbf{N M R}\left(125 \mathrm{MHz}, \mathrm{C}_{6} \mathrm{D}_{6}\right) \delta$ 170.0, 169.7, 169.4, 110.7, 79.0, 78.9, 78.0, 75.9, 71.5, 67.7, 67.6, 64.5, 30.1, 27.2, 26.5, 26.4, 26.1, 24.4, 20.5, 20.2; IR $v_{\max } / \mathrm{cm}^{-}$ ${ }^{1}$ 3511, 2928, 1744, 1372, 1234, 1050; MS m/z (ES) $455(\mathrm{M}+\mathrm{Na})^{+} ;$HRMS (ES): $\mathrm{m} / z$ calcd for $\mathrm{C}_{20} \mathrm{H}_{32} \mathrm{O}_{10} \mathrm{Na}$ : 455.1893, found 455.1895.

Stereochemical assignment of alcohol 42ax: spin-coupling constants ${ }^{3} J_{\mathrm{H}-\mathrm{H}}$ and NOE observed

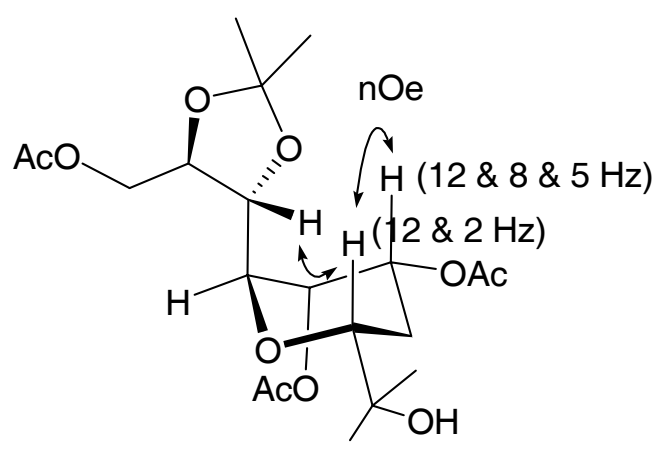

Synthesis of triacetate 42ax from triol 24ax

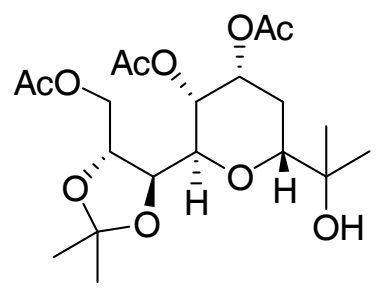

TBAF (1 M in THF, $142 \mu \mathrm{L}, 0.142 \mathrm{mmol}$ ) was added to a solution of $\mathbf{2 4 a x}(6 \mathrm{mg}, 0.014$ $\mathrm{mmol})$ in THF (2 mL). After stirring at $\mathrm{rt}$ for $24 \mathrm{~h}$, the solvent was evaporated under reduced pressure to give a residue. This material was dissolved in pyridine $(2 \mathrm{~mL})$ and treated with acetic anhydride $(0.5 \mathrm{~mL})$. After stirring at $\mathrm{rt}$ for $16 \mathrm{~h}$, ether $(3 \mathrm{~mL})$ and water $(4 \mathrm{~mL})$ were added. The organic phase was separated and the aqueous was extracted with ether $(10 \mathrm{~mL})$. The combined organic extracts were dried over $\mathrm{MgSO}_{4}$, filtered and evaporated under reduced pressure to give a pale yellow solid. This solid was purified by column chromatography (ether) to afford alcohol 42ax (5 mg, 80\%) as a colorless oil. 


\section{Synthesis of triacetates 42eq and 42ax from a mixture of alcohols 23}

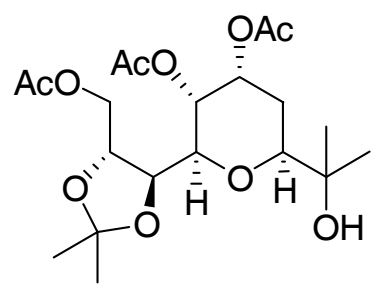

TBAF (1M in THF, $140 \mu \mathrm{L}, 0.14 \mathrm{mmol}$ ) was added to a solution of a 2:1 mixture of alcohols 23 in favor of $23 e q(15 \mathrm{mg}, 0.023 \mathrm{mmol})$ in THF $(1 \mathrm{~mL})$ at $\mathrm{rt}$. After stirring at $\mathrm{rt}$ for 24 $\mathrm{h}$, the solvent was evaporated under reduced pressure to give a residue. This material was dissolved in pyridine $(2 \mathrm{~mL})$ and treated with acetic anhydride $(0.5 \mathrm{~mL})$. After stirring at $\mathrm{rt} 16 \mathrm{~h}$. ether $(3 \mathrm{~mL})$ and water $(4 \mathrm{~mL})$ were added. The organic phase was separated and the aqueous was extracted with ether $(10 \mathrm{~mL})$. The combined organic extracts were dried over $\mathrm{MgSO}_{4}$, filtered and evaporated under reduced pressure to give the crude material, which was purified by column chromatography (ether) to afford alcohol 42eq (4 mg, 40\%) and alcohol 42ax (2 mg, $20 \%$ ) as colorless oils. 


\section{Conformational Analysis of Model Pyranosyl Radical Intermediate 26}<smiles>C[C@@H]1OCCC2OCOC21</smiles>

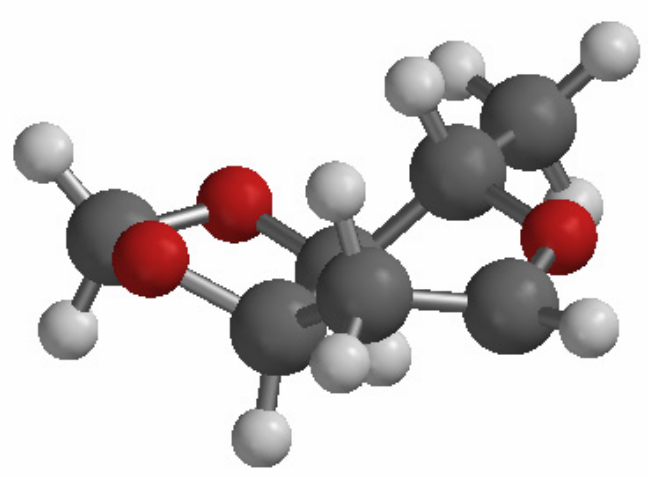

trans configuration $(0.0 \mathrm{kcal} / \mathrm{mol})$

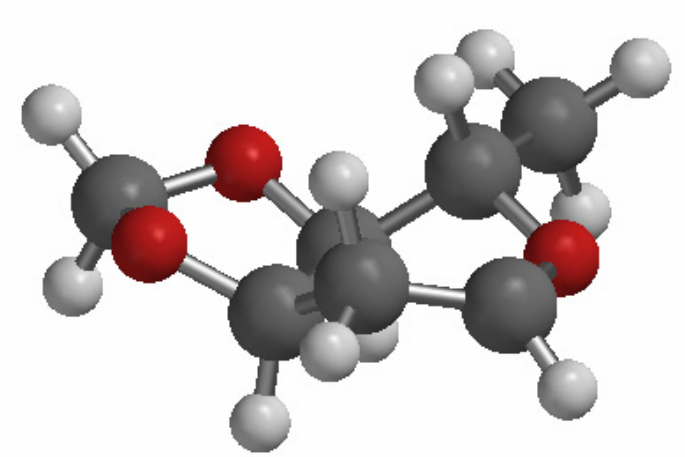

cis configuration $(0.2 \mathrm{kcal} / \mathrm{mol})$ 
trans configuration, boat conformation

Coordinates (Angstroms)

\begin{tabular}{rlrrr} 
ATOM & \multicolumn{1}{c}{$\mathrm{X}$} & $\mathrm{Y}$ & $\mathrm{Z}$ \\
& & & & \\
1 & $\mathrm{C}$ & 0.032503 & -0.580117 & -0.478860 \\
2 & $\mathrm{O}$ & -1.531570 & 1.255328 & -0.206912 \\
3 & $\mathrm{C}$ & 0.864368 & 1.785040 & 0.072500 \\
4 & $\mathrm{C}$ & -0.499888 & 2.135563 & -0.420547 \\
5 & $\mathrm{C}$ & 1.246752 & 0.394223 & -0.452999 \\
6 & $\mathrm{C}$ & -1.158625 & -0.033284 & 0.316602 \\
7 & $\mathrm{H}$ & 1.585550 & 2.541263 & -0.253489 \\
8 & $\mathrm{H}$ & -0.846600 & 3.156220 & -0.536357 \\
9 & $\mathrm{H}$ & -0.832728 & 0.088249 & 1.359546 \\
10 & $\mathrm{H}$ & -0.283146 & -0.790302 & -1.510152 \\
11 & $\mathrm{H}$ & 0.924974 & 1.747322 & 1.175907 \\
12 & $\mathrm{H}$ & 1.684161 & 0.469175 & -1.458720 \\
13 & $\mathrm{O}$ & 2.167598 & -0.247075 & 0.432102 \\
14 & $\mathrm{O}$ & 0.513722 & -1.771413 & 0.145743 \\
15 & $\mathrm{C}$ & 1.917194 & -1.620804 & 0.279119 \\
16 & $\mathrm{C}$ & -2.386909 & -0.923660 & 0.256872 \\
17 & $\mathrm{H}$ & -2.149956 & -1.910739 & 0.664879 \\
18 & $\mathrm{H}$ & -2.723373 & -1.041569 & -0.779211 \\
19 & $\mathrm{H}$ & -3.205060 & -0.486298 & 0.837463 \\
20 & $\mathrm{H}$ & 2.426919 & -2.016864 & -0.617534 \\
21 & $\mathrm{H}$ & 2.254114 & -2.150258 & 1.174048
\end{tabular}

Point Group: c1 Number of degrees of freedom: 57

Energy is -498.953711897

Spartan '02 Quantum Mechanics Program: (PC/x86) Release 116

Job type: Geometry optimization.

Method: UB3LYP

Basis set: 6-31G(D)

Number of shells: 62

Number of basis functions: 172 
cis configuration, boat conformation

Coordinates (Angstroms)

$\begin{array}{rrrrc} & \text { ATOM } & \mathrm{X} & \mathrm{Y} & \mathrm{Z} \\ & & & & \\ 1 & \mathrm{C} & 0.005336 & -0.562394 & -0.454524 \\ 2 & \mathrm{O} & -1.452018 & 1.330631 & -0.186502 \\ 3 & \mathrm{C} & 0.943408 & 1.711774 & 0.287334 \\ 4 & \mathrm{C} & -0.411390 & 2.222879 & -0.088492 \\ 5 & \mathrm{C} & 1.245800 & 0.384704 & -0.429660 \\ 6 & \mathrm{C} & -1.182265 & 0.014149 & 0.325279 \\ 7 & \mathrm{H} & 1.705343 & 2.463744 & 0.060208 \\ 8 & \mathrm{H} & -0.895950 & 0.085548 & 1.383749 \\ 9 & \mathrm{H} & -0.308857 & -0.762944 & -1.489180 \\ 10 & \mathrm{H} & 1.017829 & 1.506638 & 1.366995 \\ 11 & \mathrm{H} & 1.595144 & 0.572321 & -1.454539 \\ 12 & \mathrm{O} & 2.229480 & -0.360271 & 0.293682 \\ 13 & \mathrm{O} & 0.456654 & -1.763672 & 0.169705 \\ 14 & \mathrm{C} & 1.869604 & -1.711258 & 0.138671 \\ 15 & \mathrm{C} & -2.454063 & -0.803342 & 0.185552 \\ 16 & \mathrm{H} & -2.285245 & -1.818824 & 0.556385 \\ 17 & \mathrm{H} & -2.759412 & -0.857899 & -0.865366 \\ 18 & \mathrm{H} & -3.267288 & -0.346710 & 0.757987 \\ 19 & \mathrm{H} & 2.246145 & -2.111001 & -0.820464 \\ 20 & \mathrm{H} & 2.268786 & -2.289887 & 0.975547 \\ 21 & \mathrm{H} & -0.567040 & 3.095813 & -0.712369\end{array}$

Point Group: c1 Number of degrees of freedom: 57

Energy is -498.953387953

Spartan '02 Quantum Mechanics Program: (PC/x86) Release 116

Job type: Geometry optimization.

Method: UB3LYP

Basis set: 6-31G(D)

Number of shells: 62

Number of basis functions: 172 